\title{
Aminohydroxymethylene $\left(\mathrm{H}_{2} \mathrm{~N}-\ddot{\mathrm{C}}-\mathrm{OH}\right)$ - The Simplest Aminooxycarbene
}

Bastian Bernhardt," Marcel Ruth," Hans Peter Reisenauer, and Peter R. Schreiner*

Institute of Organic Chemistry, Justus Liebig University, Heinrich-Buff-Ring 17, 35392 Giessen, Germany

*prs@uni-giessen.de

\# These authors contributed equally.

\section{Table of Contents}

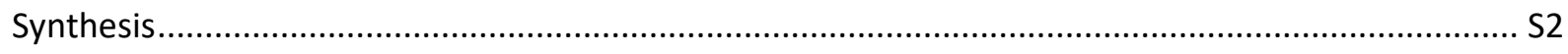

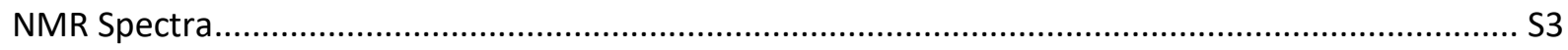

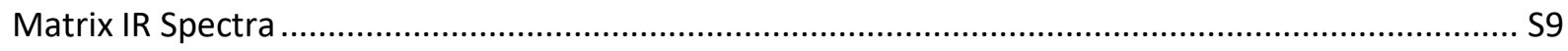

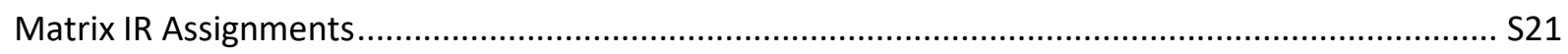

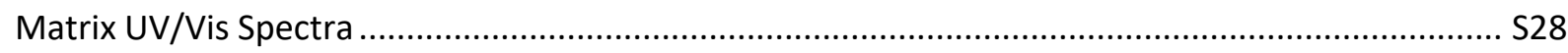

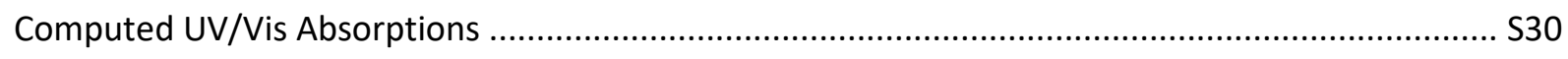

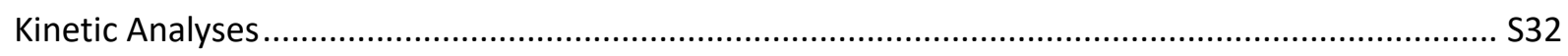

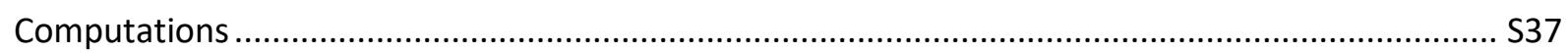

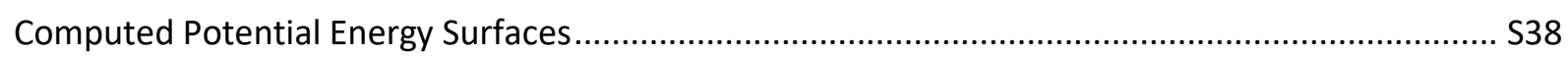

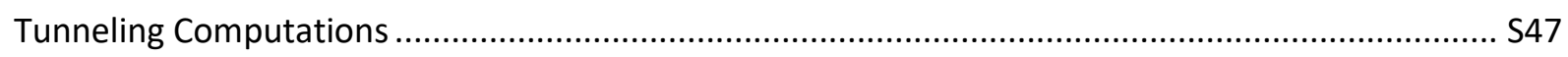

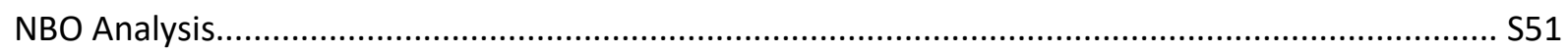

Cartesian Coordinates of Computationally Optimized Geometries.............................................. S52

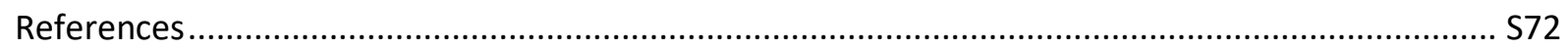

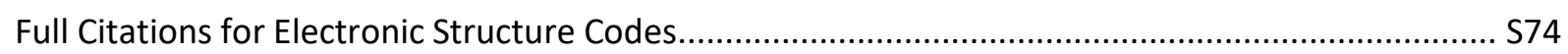




\section{Synthesis}

Oxalic acid monoamide (2):

Oxalic acid monoamide (2) was purchased from TCl Deutschland GmbH (purity > 97\%) and used for matrix isolation experiments without further purification.

${ }^{1} \mathrm{H}$ NMR $\left(400 \mathrm{MHz}, \mathrm{DMSO}-d_{6}\right): \delta=13.62(\mathrm{~s}, 1 \mathrm{H}), 8.11(\mathrm{~s}, 1 \mathrm{H}), 7.80(\mathrm{~s}, 1 \mathrm{H}) \mathrm{ppm}$.

${ }^{2} \mathrm{H}$ NMR $\left(61 \mathrm{MHz}, \mathrm{DMSO}-d_{6}\right)$ : blank spectrum.

${ }^{13} \mathrm{C}\left\{{ }^{1} \mathrm{H}\right\}$ NMR $\left(101 \mathrm{MHz}\right.$, DMSO- $\left.d_{6}\right): \delta=162.5,160.4 \mathrm{ppm}$.

Perdeuterated oxalic acid monoamide $\left(\boldsymbol{d}_{\mathbf{3}}-\mathbf{2}\right)$ :

Ca. $775 \mathrm{mg}$ (8.71 mmol) 2 were dissolved in ca. $25 \mathrm{~mL}(1.25 \mathrm{~mol}) \mathrm{D}_{2} \mathrm{O}$ and stirred for $15 \mathrm{~min}$. The solvent was removed under reduced pressure to yield a colorless solid. This procedure was repeated ten times. Complete deuteration was confirmed by the absence of $\mathrm{OH}$ and $\mathrm{NH}$ bands in matrix IR spectra. Note that NMR spectra of solutions of $\boldsymbol{d}_{\mathbf{3}} \mathbf{- 2}$ in DMSO show small proton signals presumably due to exchange with $\mathrm{H}_{2} \mathrm{O}$ residues in DMSO (vide infra).

${ }^{1} \mathrm{H}$ NMR $\left(400 \mathrm{MHz}, \mathrm{DMSO}-d_{6}\right): \delta=8.13(\mathrm{~s}, 1 \mathrm{H}), 7.82(\mathrm{~s}, 1 \mathrm{H}) \mathrm{ppm}$.

${ }^{2} \mathrm{H}$ NMR (61 MHz, DMSO- $\left.d_{6}\right): \delta=8.11,7.79 \mathrm{ppm}$.

${ }^{13} \mathrm{C}\left\{{ }^{1} \mathrm{H}\right\}$ NMR $\left(101 \mathrm{MHz}\right.$, DMSO- $\left.d_{6}\right): \delta=162.4(\mathrm{~m}), 160.2(\mathrm{~m}) \mathrm{ppm}$. 


\section{NMR Spectra}

Oxalic acid monoamide (2):
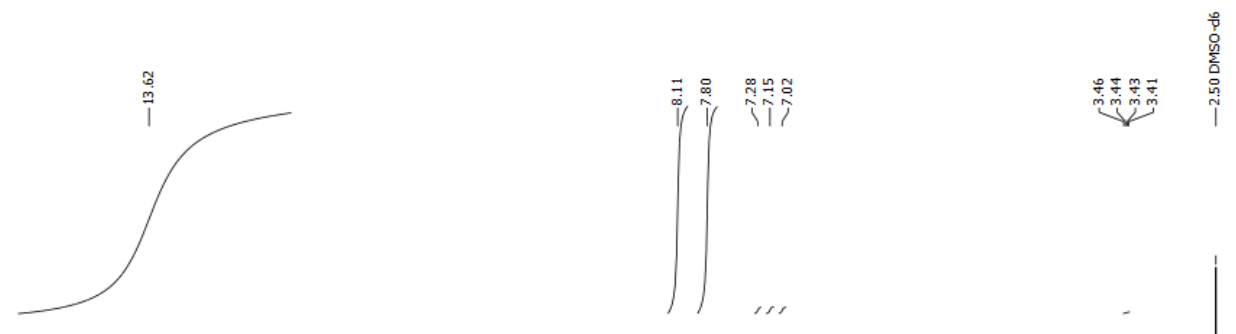<smiles>NC(=O)C(=O)O</smiles>

2

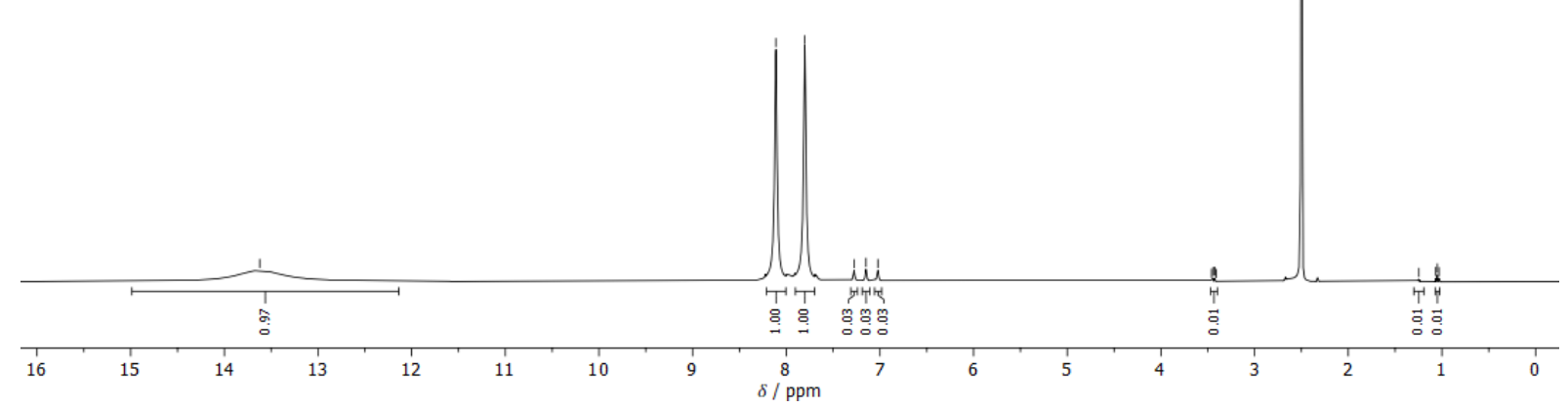

Figure S1: ${ }^{1} \mathrm{H}$ NMR spectrum of oxalic acid monoamide (2). Peaks at 13.62, 8.11, and $7.80 \mathrm{ppm}$ can be assigned to 2 . A quartet at 3.44 and a triplet at $1.05 \mathrm{ppm}$ can be assigned to traces of ethanol in the sample. Peaks at 7.28, 7.15, 7.02, and $1.25 \mathrm{ppm}$ hint to traces of unknown impurities. 


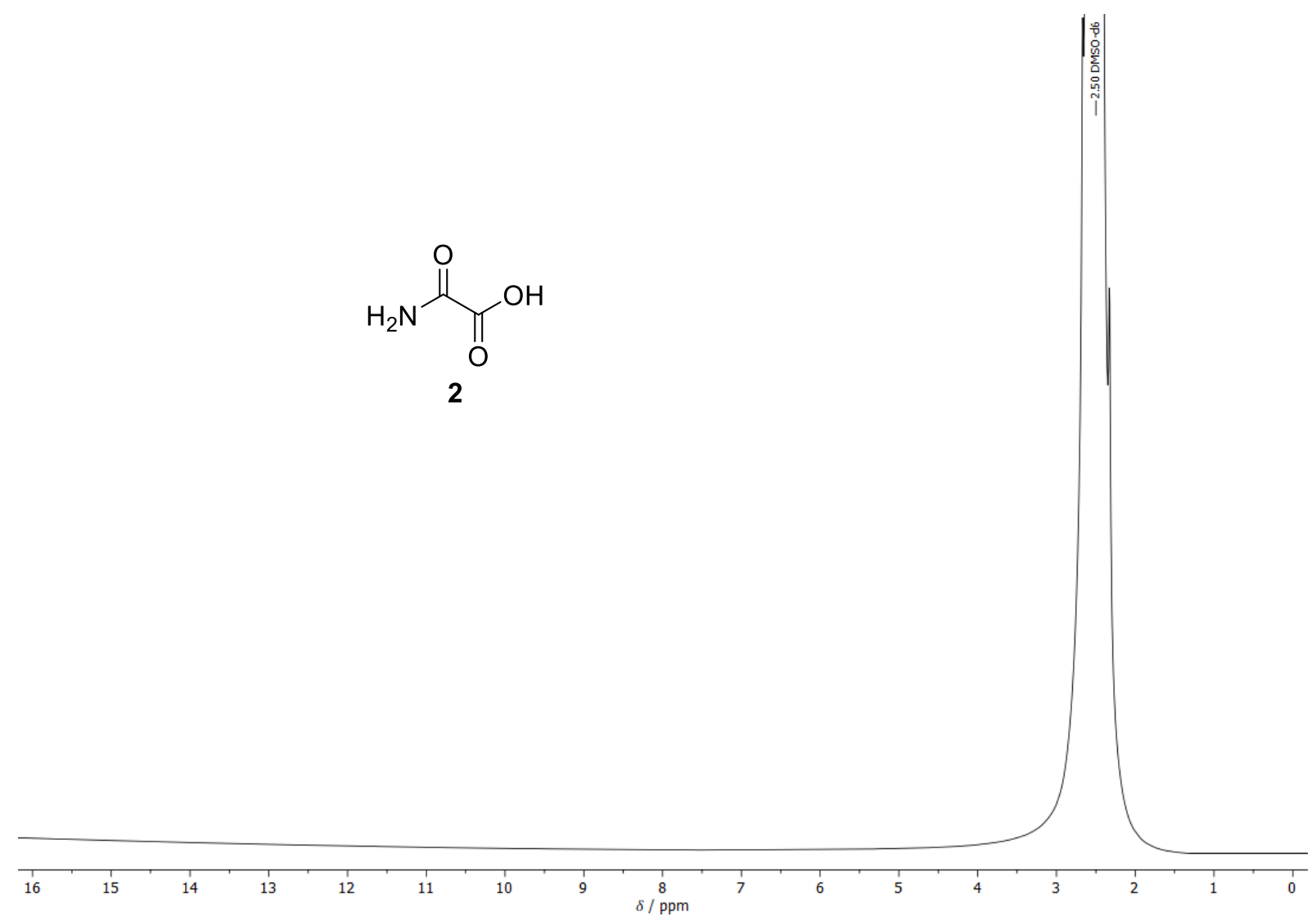

Figure S2: ${ }^{2} \mathrm{H}$ NMR spectrum of oxalic acid monoamide (2). The blank spectrum denotes the absence of any deuterated material. 

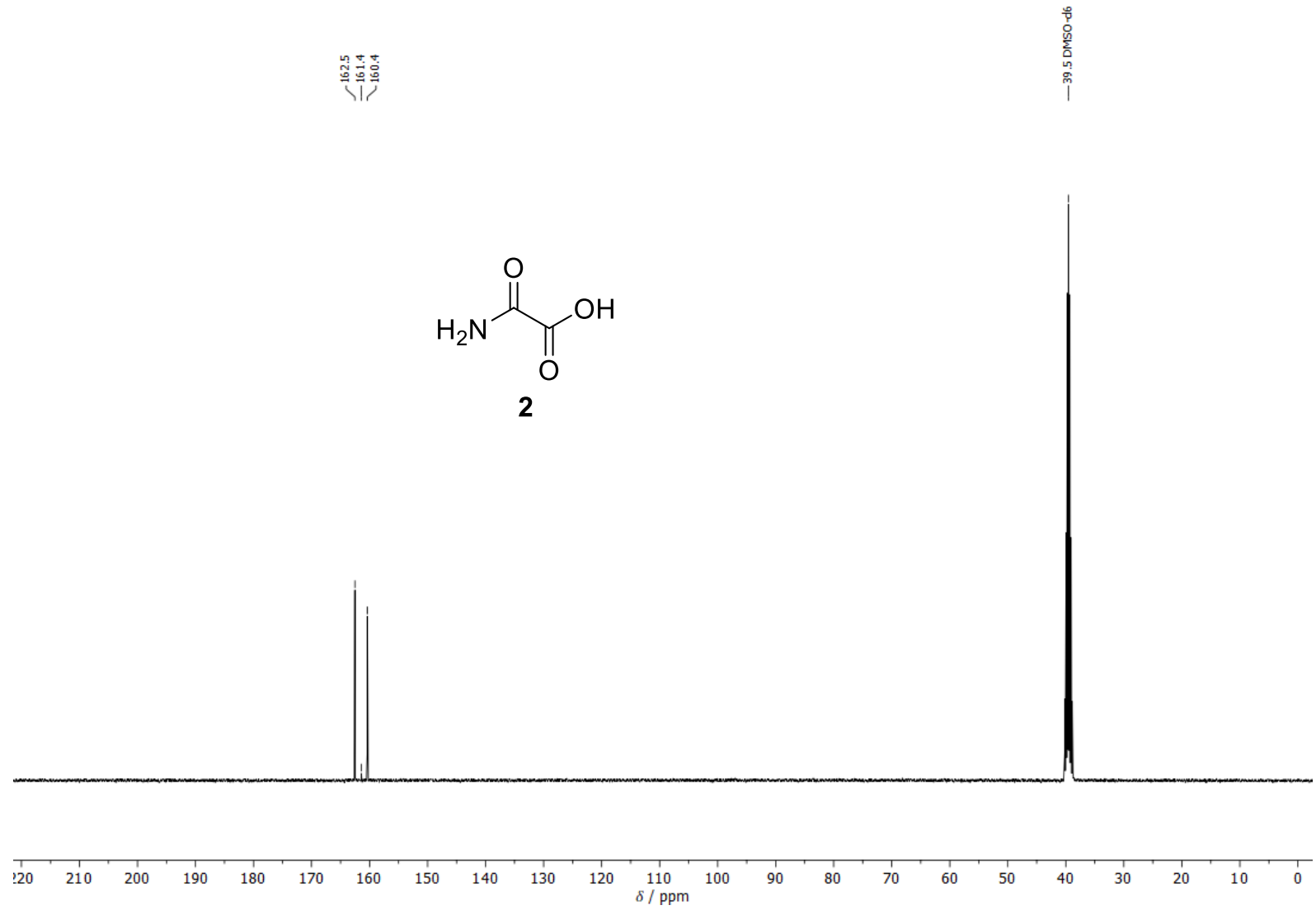

Figure S3: ${ }^{13} \mathrm{C}\left\{{ }^{1} \mathrm{H}\right\}$ NMR spectrum of oxalic acid monoamide (2). Peaks at 162.5 and $160.4 \mathrm{ppm}$ can be assigned to 2 . A small peak 161.4 ppm hints to traces of an unknown impurity in the sample. 
Perdeuterated oxalic acid monoamide $\left(\boldsymbol{d}_{\mathbf{3}}-\mathbf{2}\right)$ :<smiles>O=C([O-])C(=O)O[Na]</smiles>

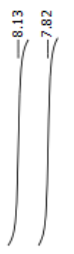

$d_{3}-2$

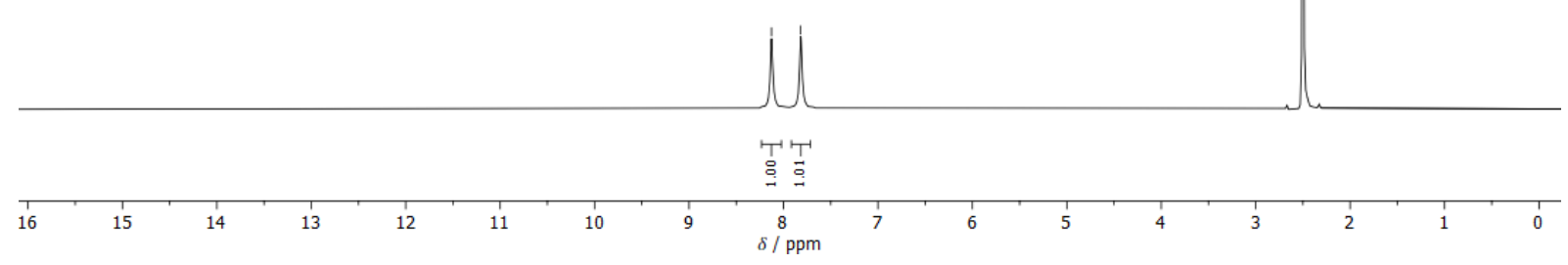

Figure S4: ${ }^{1} \mathrm{H}$ NMR spectrum of perdeuterated oxalic acid monoamide $\left(\boldsymbol{d}_{\mathbf{3}}-\mathbf{2}\right)$. Peaks at 8.13 , and $7.82 \mathrm{ppm}$ can be assigned to protonated isotopologues of $\mathbf{2}$, which presumably originate due to exchange with $\mathrm{H}_{2} \mathrm{O}$ residues in DMSO. 


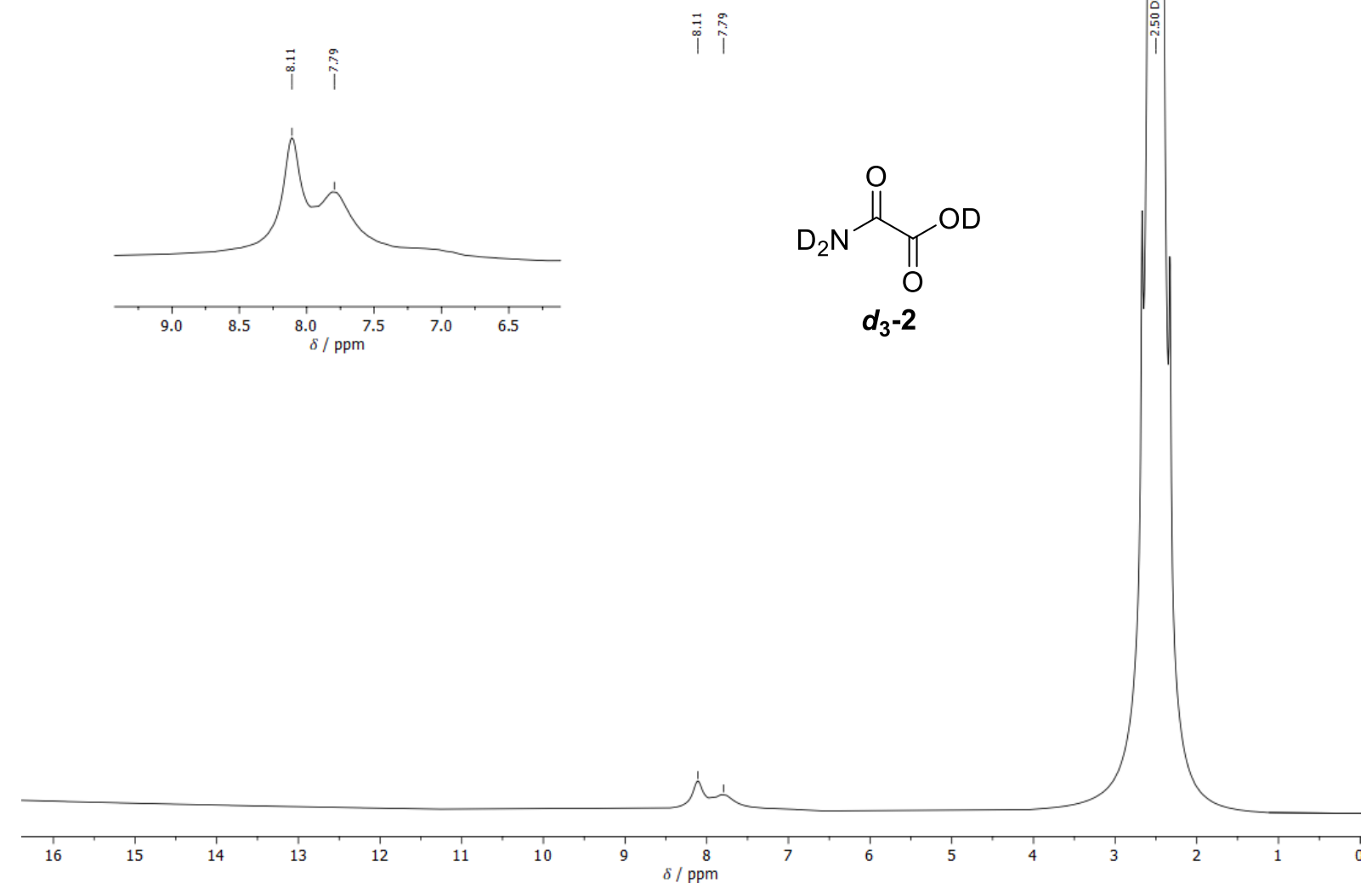

Figure S5: ${ }^{2} \mathrm{H}$ NMR spectrum of perdeuterated oxalic acid monoamide $\left(\boldsymbol{d}_{\mathbf{3}}-\mathbf{2}\right)$. Peaks at 8.11, and $7.79 \mathrm{ppm}$ can be assigned to $\boldsymbol{d}_{\mathbf{3}} \mathbf{- 2}$. 

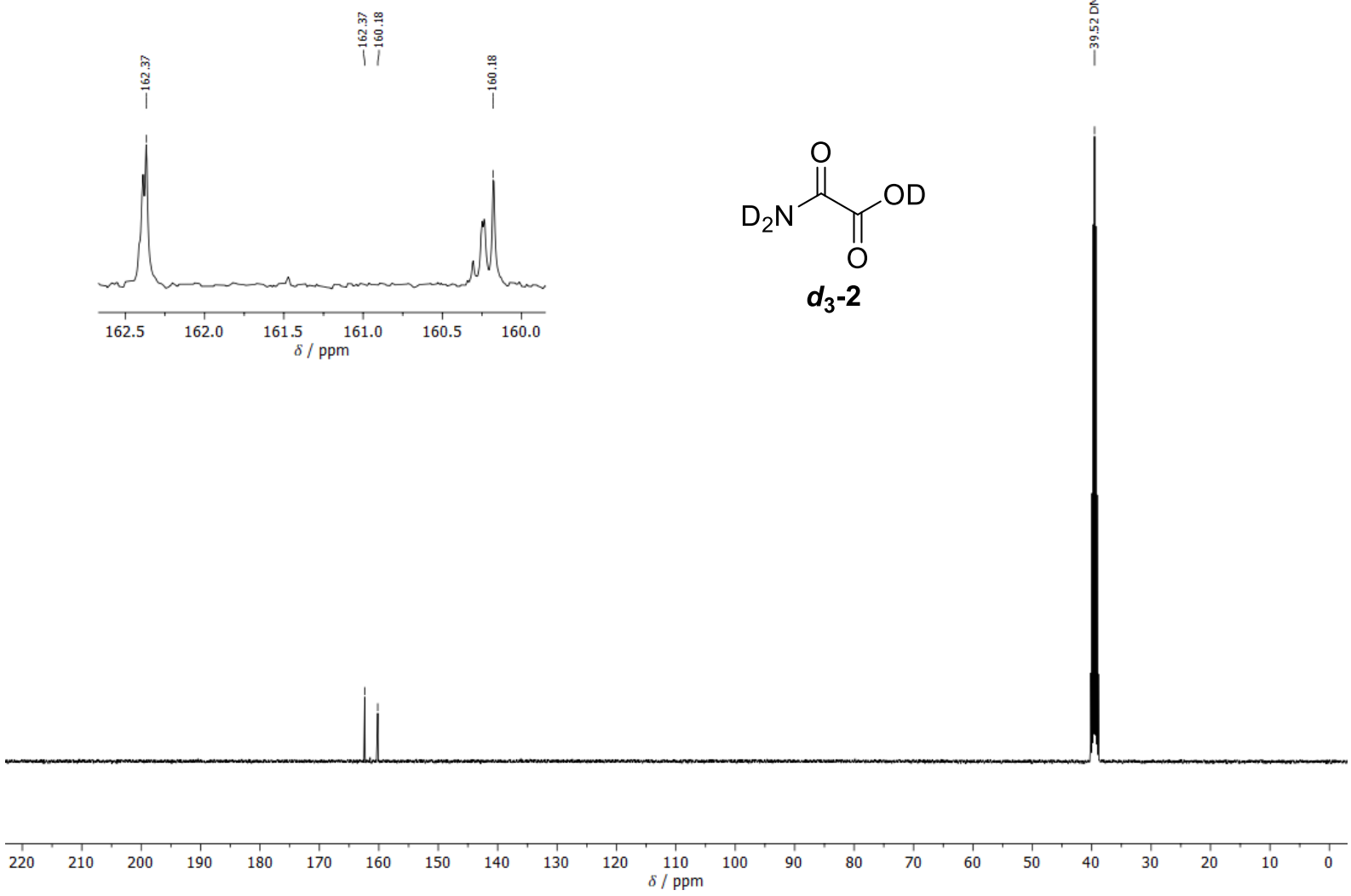

Figure S6: ${ }^{13} \mathrm{C}\left\{{ }^{1} \mathrm{H}\right\}$ NMR spectrum of perdeuterated oxalic acid monoamide $\left(\boldsymbol{d}_{\mathbf{3}}-\mathbf{2}\right)$. Peaks at 162.4 and $160.2 \mathrm{ppm}$ can be assigned to $\boldsymbol{d}_{\mathbf{3}} \mathbf{- 2}$. The coupling denotes the presence of deuterium in $\mathrm{OD}$ and $\mathrm{ND}_{2}$. 


\section{Matrix IR Spectra}

Photolysis of cis-oxalic acid monoamide (2c):

Our results on the photolysis of $\mathbf{2}$ are in complete agreement with those published elsewhere. ${ }^{1}$ We show our recorded spectra for the sake of completeness.

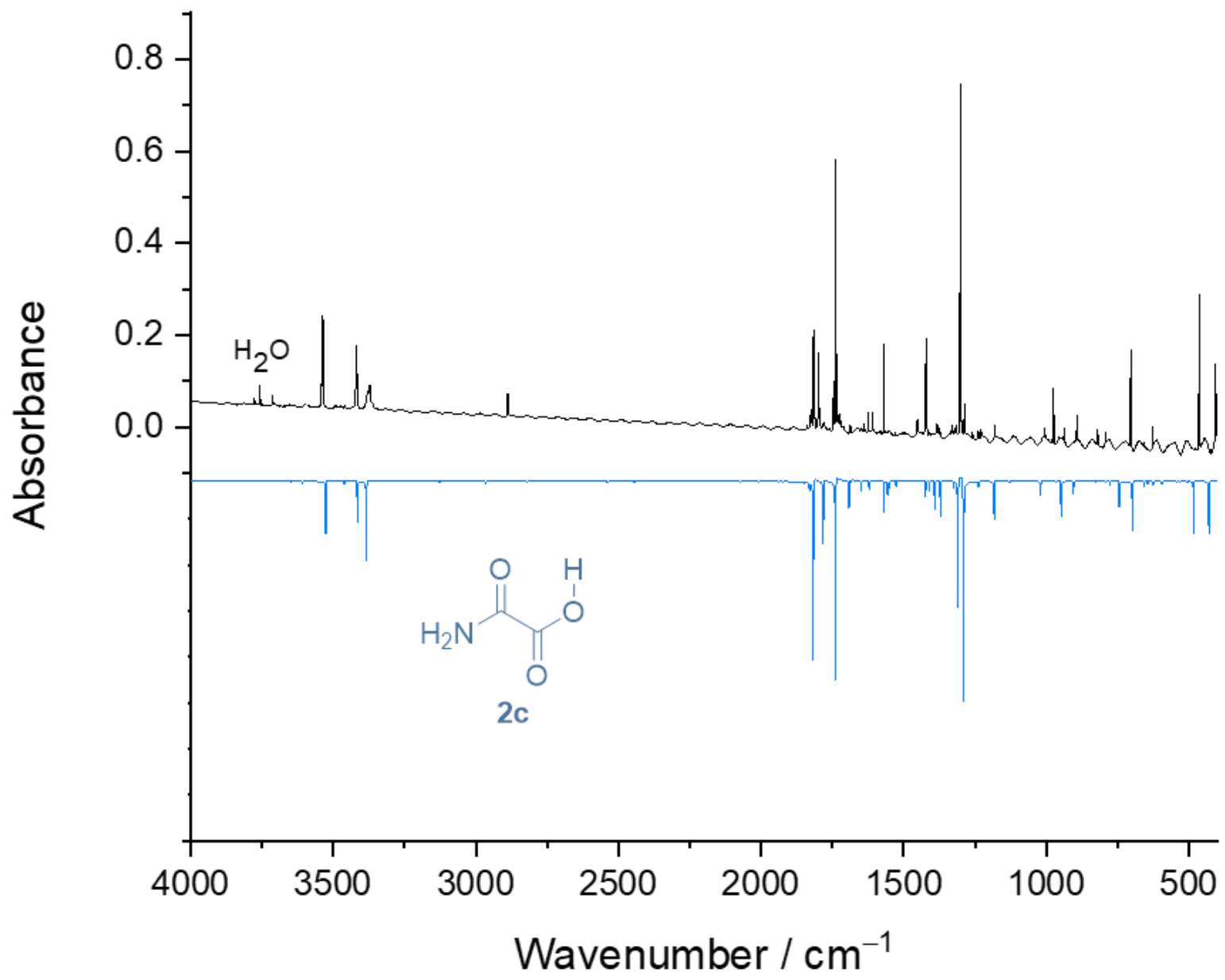

Figure S7: Matrix IR spectrum obtained after deposition of oxalic acid monoamide (2) together with an excess of $\mathrm{Ar}$ on a cold ( $3 \mathrm{~K}) \mathrm{Cs}$ l window (black) in comparison with the computed anharmonic spectrum of the energetically lowest lying cis-conformers (2c) at the B3LYP/6$311++G(3 d f, 3 p d)$ level of theory (blue). 


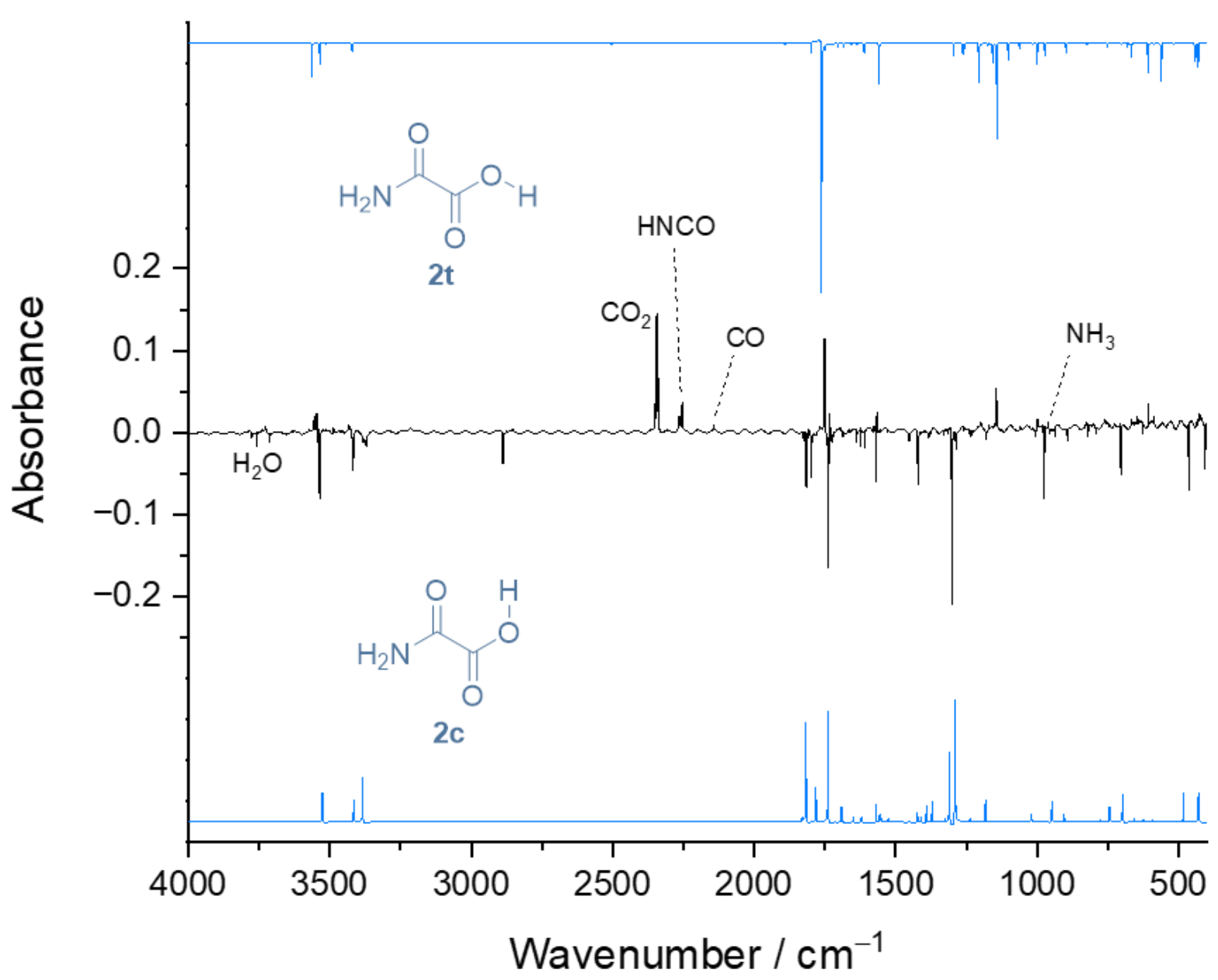

Figure S8: Black: Experimental difference matrix IR spectrum of spectra measured after deposition of 2 and irradiating the resulting matrix at $254 \mathrm{~nm}$ for $1 \mathrm{~h}$. Blue: Computed anharmonic spectra of $\mathbf{2 c}$ and $\mathbf{2 t}$ at the B3LYP/6-311++G(3df,3pd) level of theory. During irradiation $\mathbf{2 c}$ rotamerizes to $\mathbf{2 t}$ (unassigned bands) while concomitantly forming $\mathrm{CO}_{2}$, $\mathrm{HNCO}+\mathrm{H}_{2}$, and $\mathrm{CO}+\mathrm{NH}_{3}$ as additional products. 


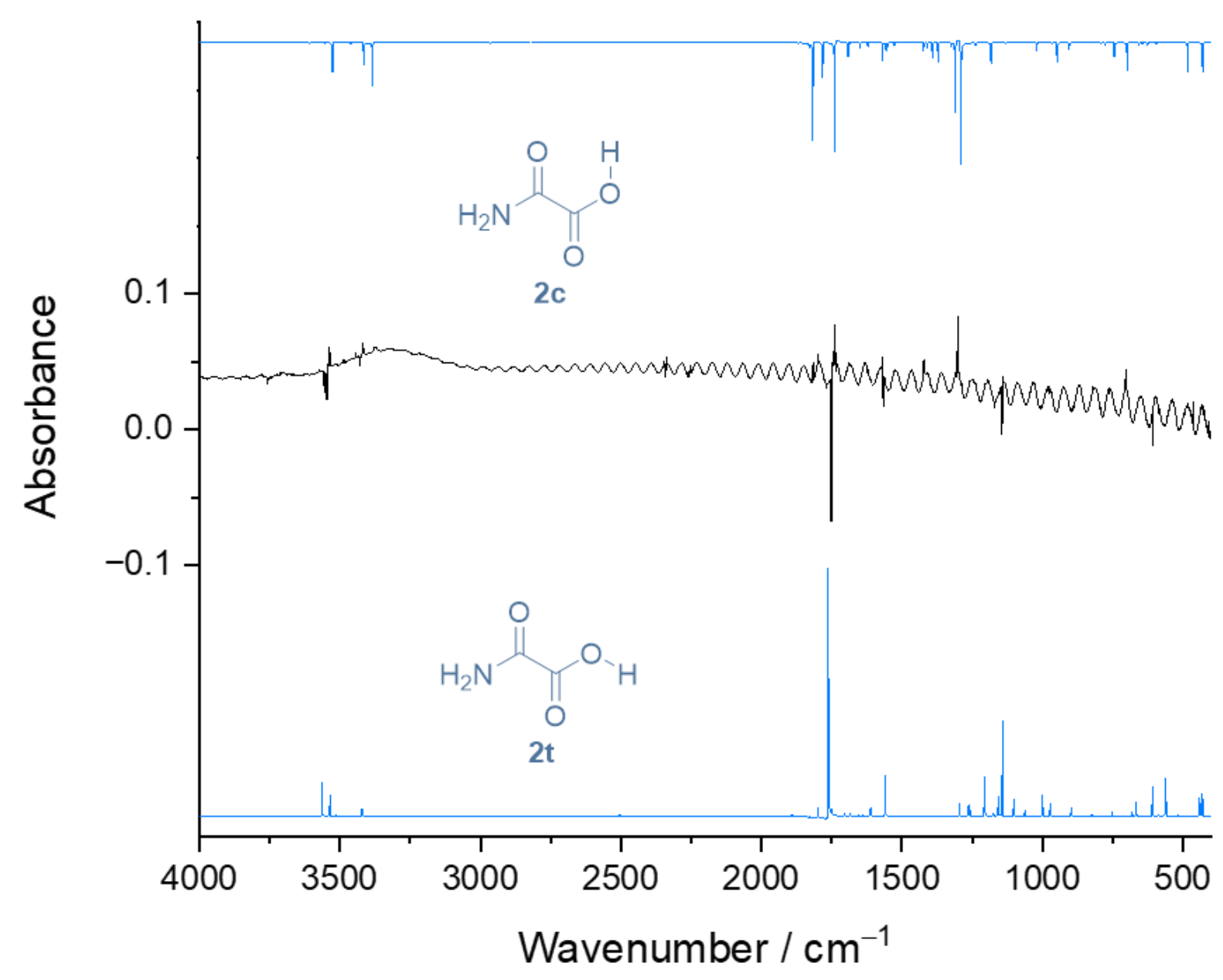

Figure 59: After irradiation at $254 \mathrm{~nm}$ the reaction $\mathbf{2 c} \rightarrow \mathbf{2 t}$ occurs (difference spectrum in Figure S8). Keeping the resulting matrix in the dark leads to the reverse reaction $\mathbf{2 t} \rightarrow \mathbf{2 c}$ via QMT. The corresponding difference spectrum is shown in black and computed anharmonic spectra at the B3LYP/6-311++G(3df,3pd) level of theory of both conformers of $\mathbf{2}$ are displayed in blue. 


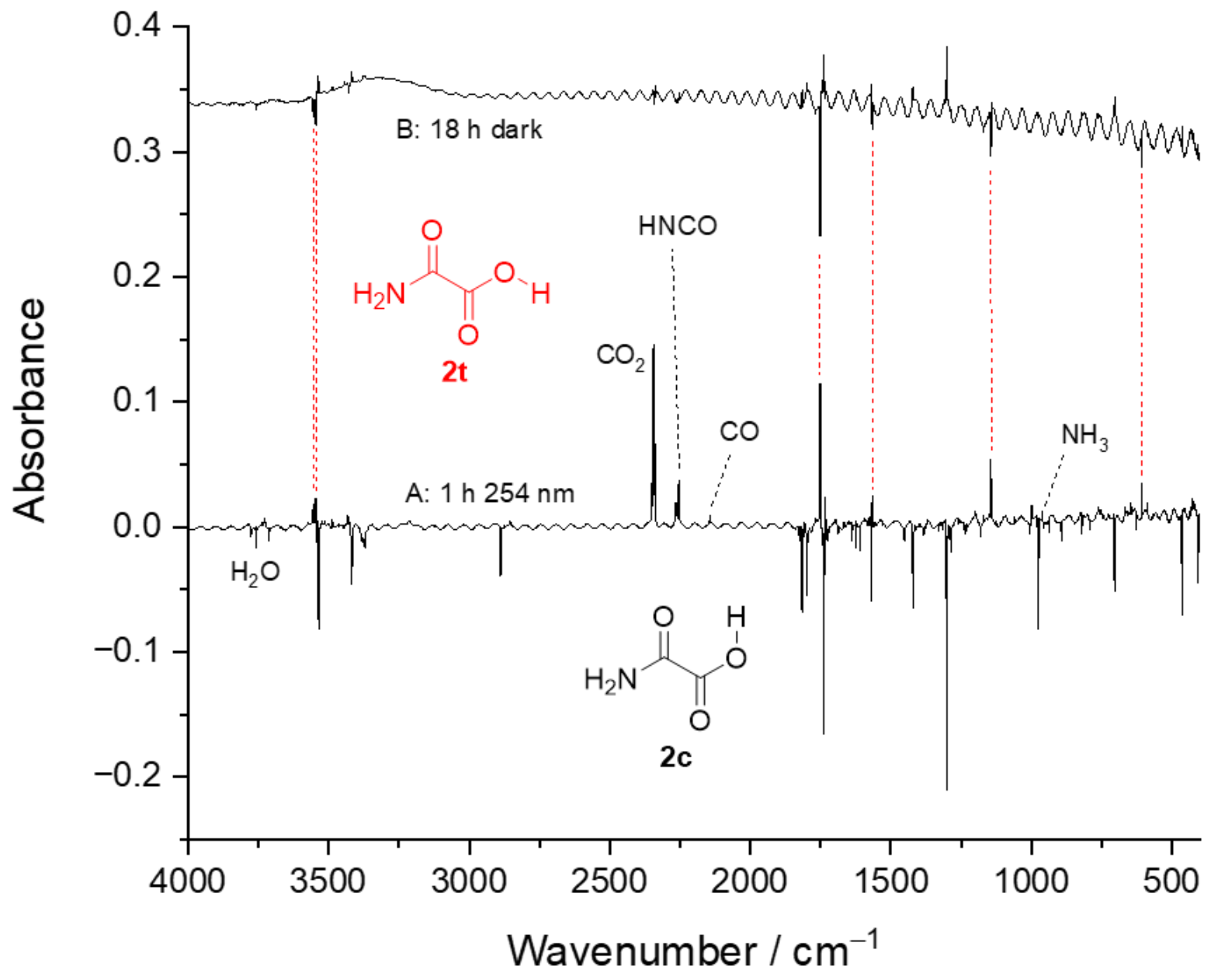

Figure S10: Comparison of the difference spectra obtained after irradiation at $254 \mathrm{~nm}$ (trace $A, \mathbf{2 c} \rightarrow \mathbf{2 t}$, difference spectrum from Figure S8) and after keeping the matrix in the dark (trace $B, \mathbf{2 t} \rightarrow \mathbf{2 c}$, difference spectrum from Figure S9). While the bands of $\mathbf{2 t}$ (red dashed lines) vanish completely in the course of $18 \mathrm{~h}$, bands of $2 \mathrm{c}$ do not reach their initial intensities. This is due to the formation of $\mathrm{CO}_{2}, \mathrm{HNCO}, \mathrm{CO}$, and $\mathrm{NH}_{3}$ as additional photolysis products of $\mathbf{2 c}$. 


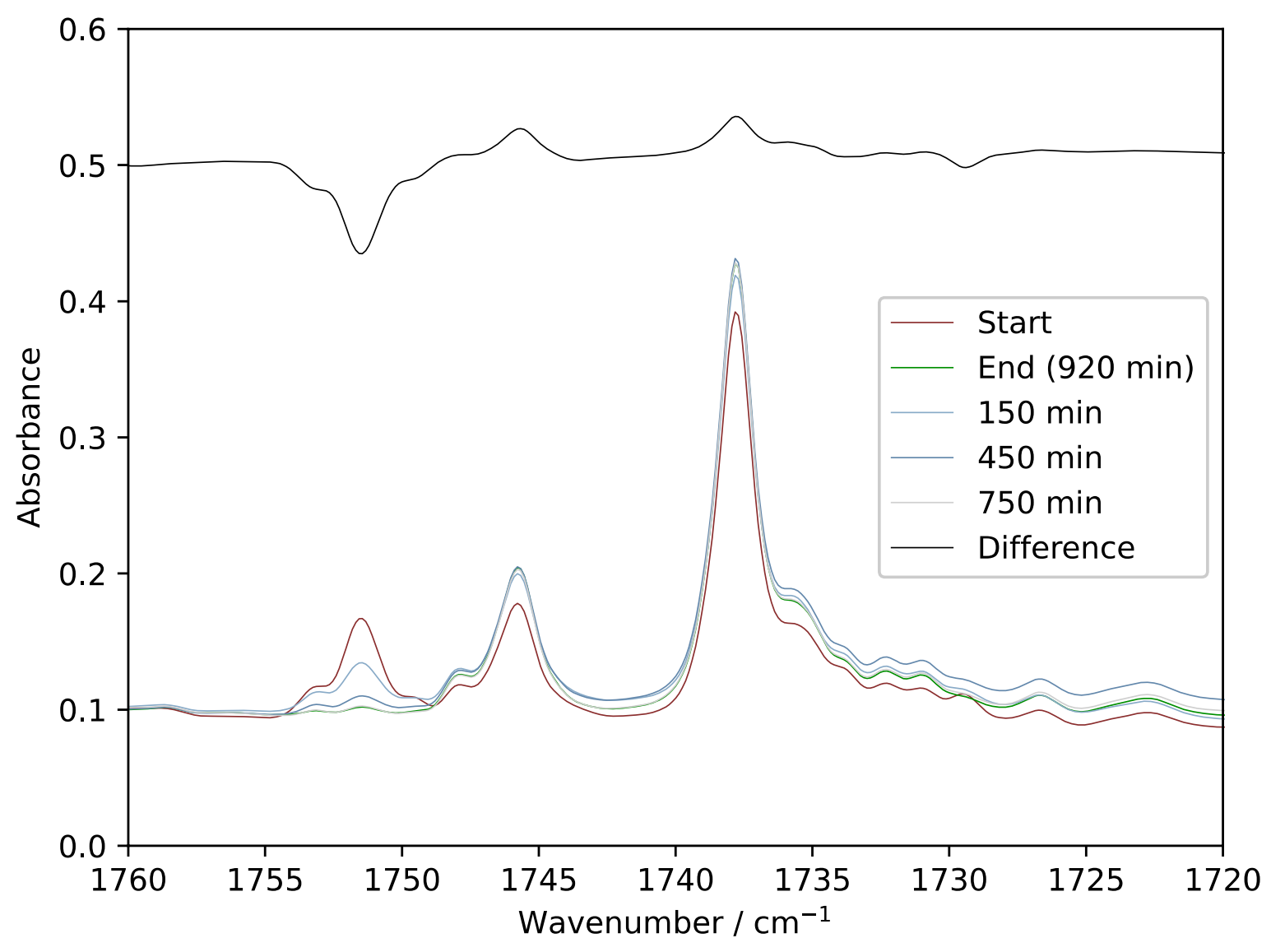

Figure S11: Detail of spectra measured during the reaction QMT $2 t \rightarrow \mathbf{2 c}$. Bands of $\mathbf{2 t}$ decrease while bands of $\mathbf{2 t}$ increase in intensity over time. We performed our kinetic analysis (vide infra) in this spectral range. 
Pyrolysis of cis-oxalic acid monoamide (2c):

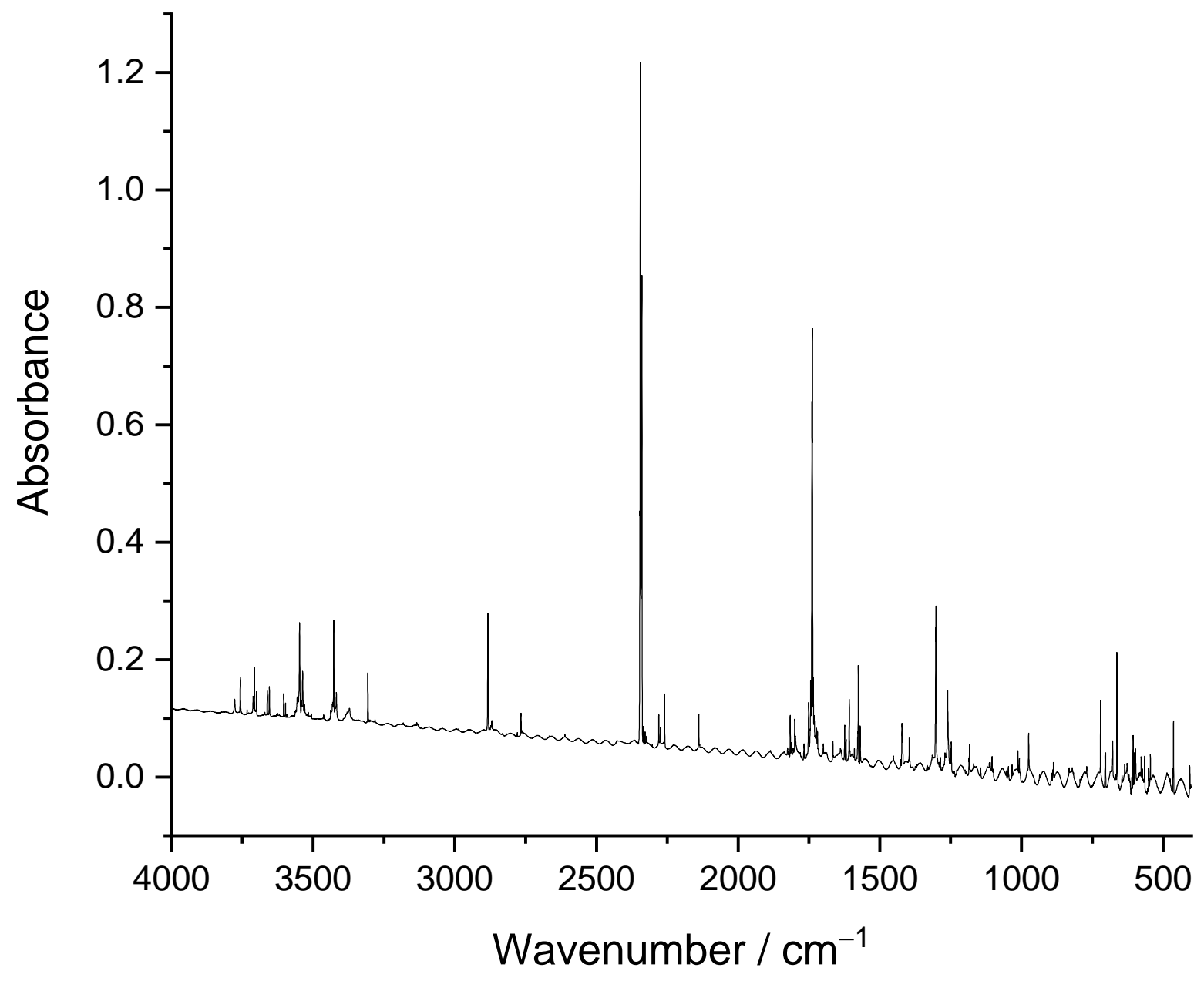

Figure S12: Typical matrix IR spectrum obtained after pyrolysis of oxalic acid monoamide (2). Detailed regions and assignments are shown in Figures S13 to S15. 


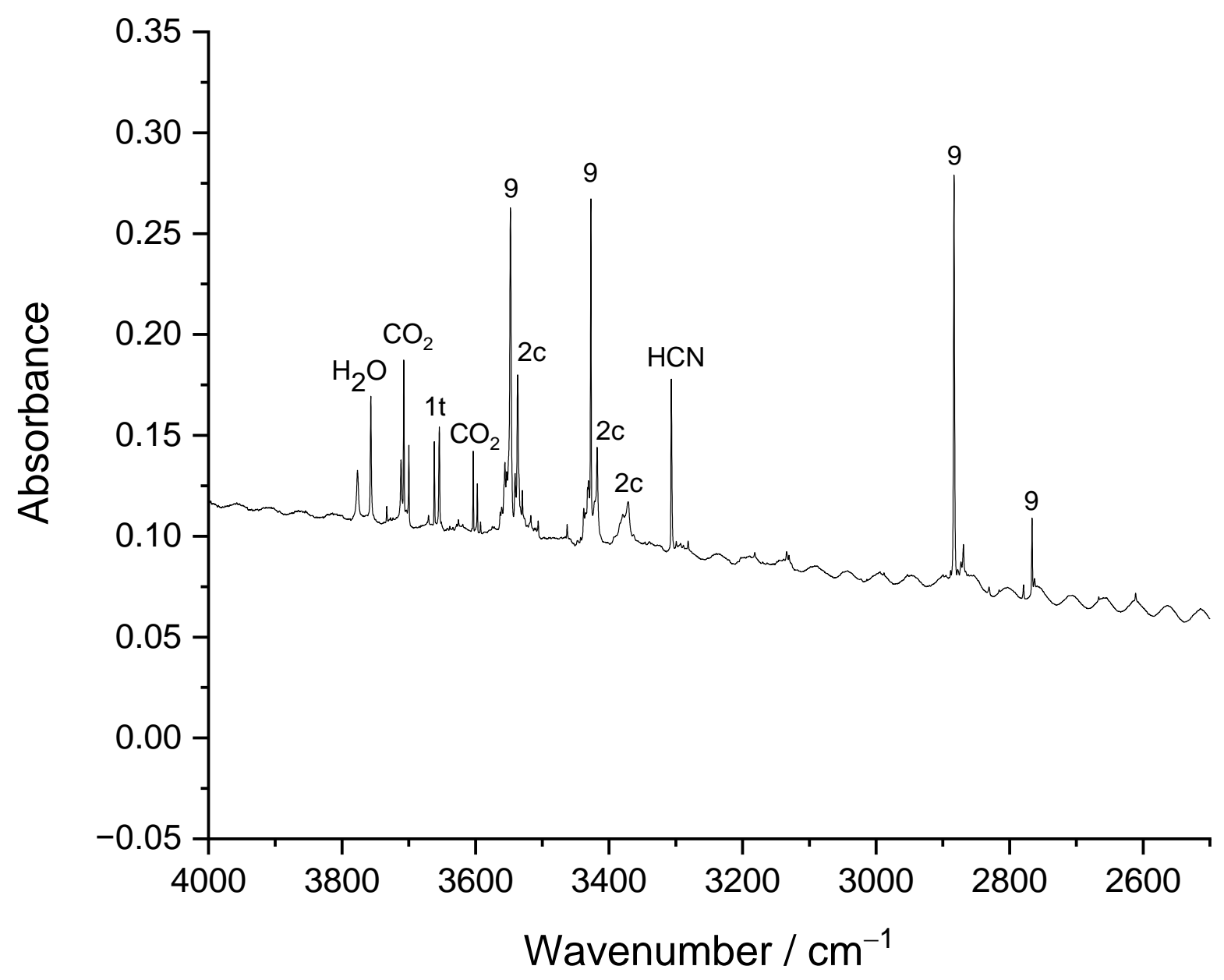

Figure S13: Detail of the 2500 to $4000 \mathrm{~cm}^{-1}$ spectral region of the pyrolysis spectrum depicted in Figure S12. 


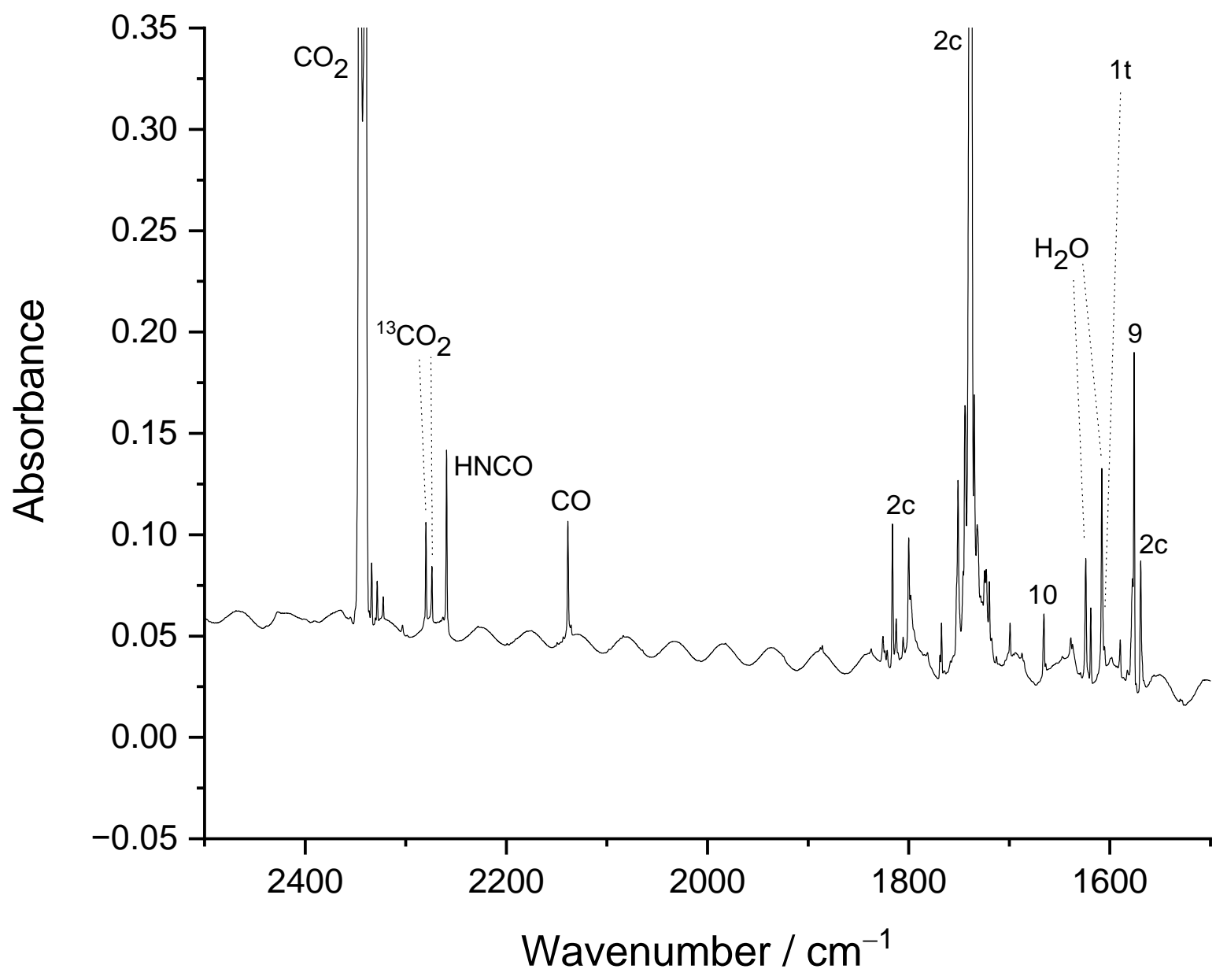

Figure S14: Detail of the 1500 to $2500 \mathrm{~cm}^{-1}$ spectral region of the pyrolysis spectrum depicted in Figure S12. 


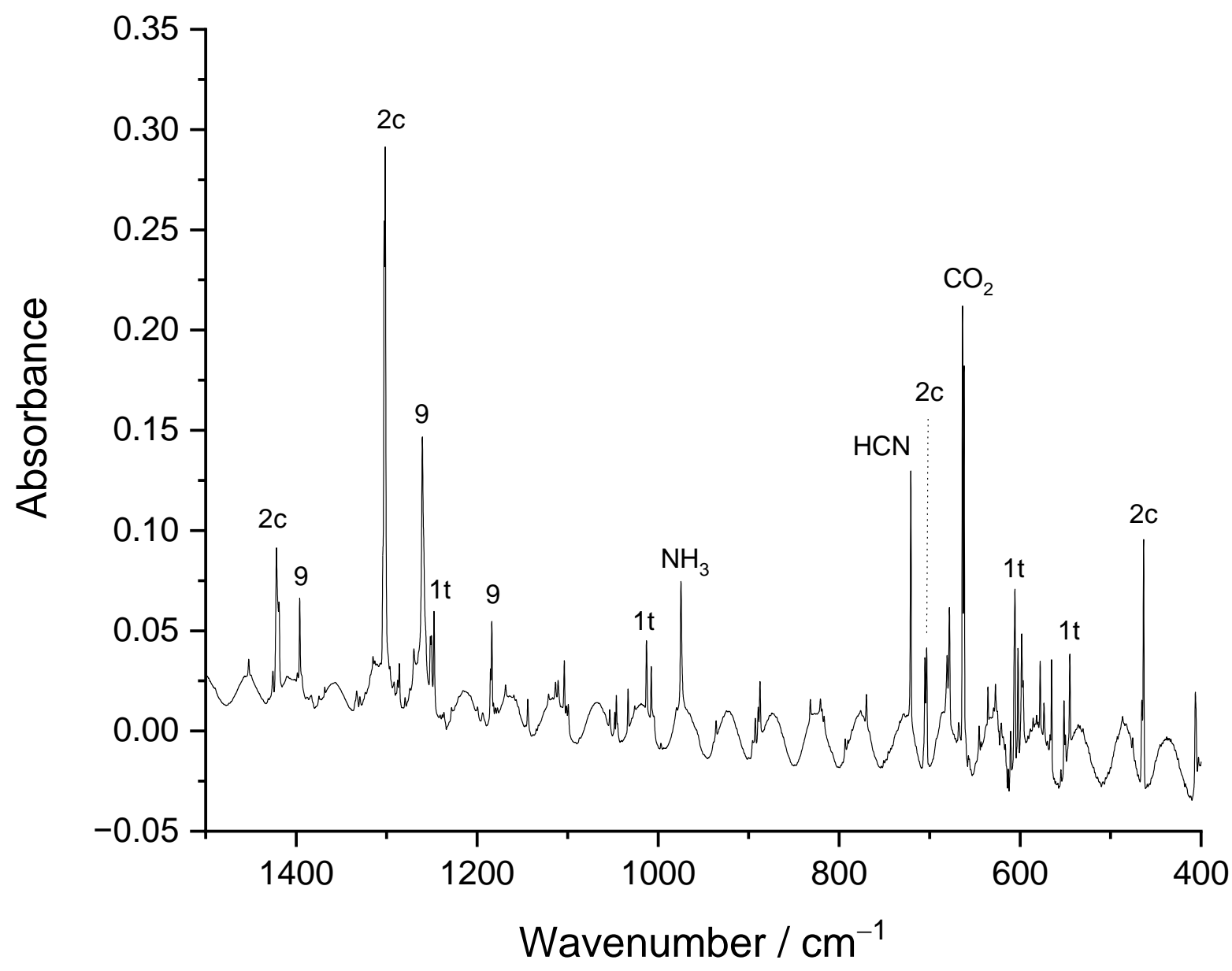

Figure S15: Detail of the 400 to $1500 \mathrm{~cm}^{-1}$ spectral region of the pyrolysis spectrum depicted in Figure S12. 
Photolysis of perdeuterated cis-oxalic acid monoamide $\left(\boldsymbol{d}_{\mathbf{3}}-\mathbf{2 c}\right)$ :

Our results on the photolysis of $\boldsymbol{d}_{\mathbf{3}}-\mathbf{2}$ are in complete agreement with those published elsewhere. ${ }^{1}$ We show our recorded spectra for the sake of completeness.

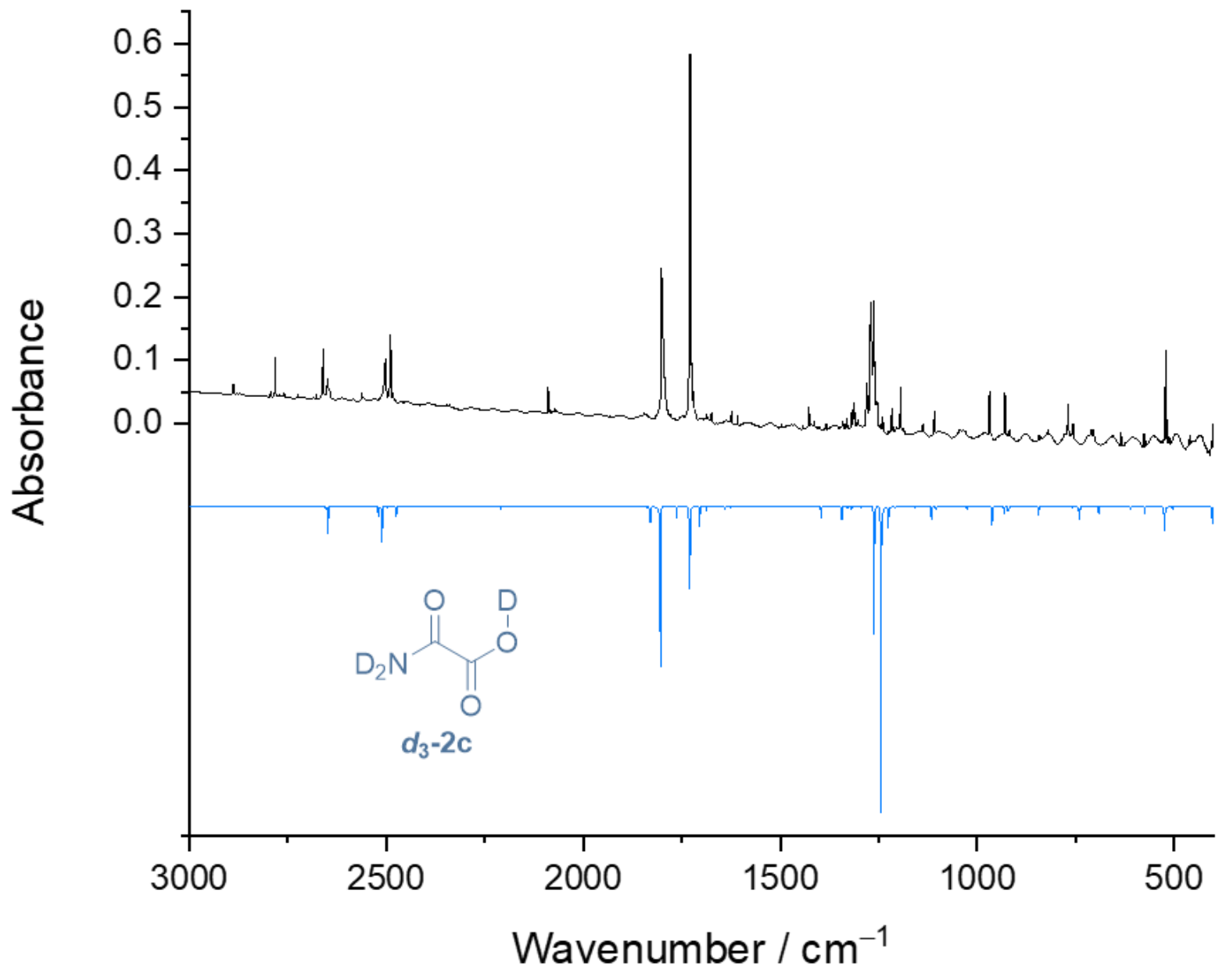

Figure S16: Matrix IR spectrum obtained after deposition of perdeuterated oxalic acid monoamide $\left(\boldsymbol{d}_{3}-\mathbf{2}\right)$ together with an excess of $\mathrm{Ar}$ on a cold $(3 \mathrm{~K}) \mathrm{Csl}$ window (black) in comparison with the anharmonic spectrum of the energetically lowest lying cis-conformers $\left(\boldsymbol{d}_{3}-2 \mathrm{c}\right)$ computed at the B3LYP/6-311++G(3df,3pd) level of theory (blue). 


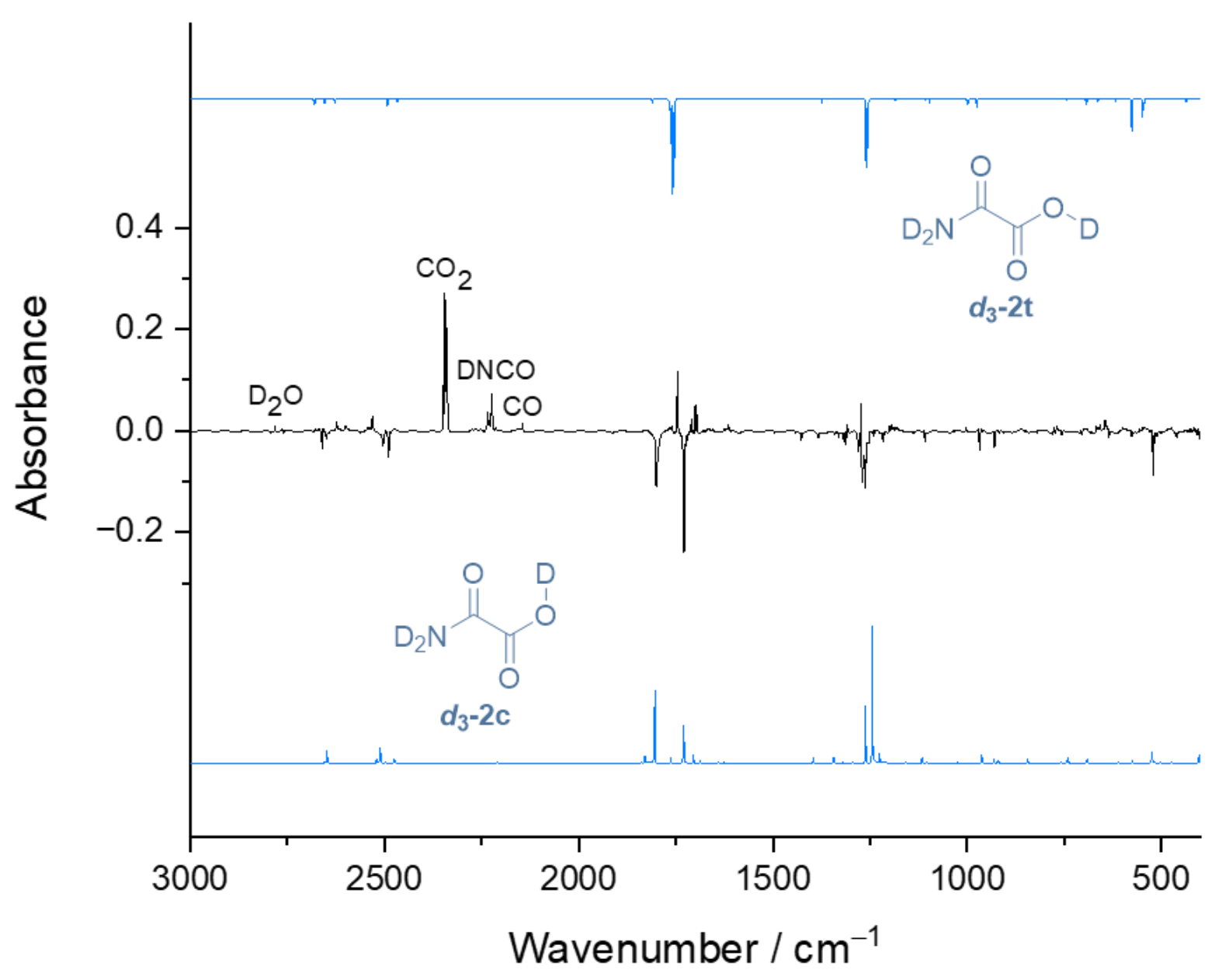

Figure S17: After irradiation at $254 \mathrm{~nm}$ for $1 \mathrm{~h}$ the reaction $\boldsymbol{d}_{\mathbf{3}}-\mathbf{2 c} \rightarrow \boldsymbol{d}_{\mathbf{3}} \mathbf{- 2 t}$ occurs. The corresponding difference spectrum is shown in black and computed anharmonic spectra at the B3LYP/6-311++G(3df,3pd) level of theory of both conformers of $\boldsymbol{d}_{\mathbf{3}} \mathbf{- 2}$ are displayed in blue. In contrast to the undeuterated case the reverse reaction is not observed after keeping the matrix for $24 \mathrm{~h}$ in the dark. $\mathrm{CO}_{2}, \mathrm{CO}+\mathrm{ND}_{3}$, and $\mathrm{DNCO}+\mathrm{D}_{2}$ form as additional photoproducts. 
Pyrolysis of a mixture of trideuterated cis-oxalic acid monoamide $\left(\boldsymbol{d}_{\mathbf{3}}-2 \mathrm{c}\right)$ and dideuterated cisoxalic acid monoamide $\left(\boldsymbol{d}_{\mathbf{2}}-\mathbf{2} \boldsymbol{c}\right)$ :

The abundant isotopologues of aminohydroxymethylene (1) in this experiment are $\mathrm{D}_{2} \mathrm{~N}-\ddot{\mathrm{C}}-\mathrm{OD}$ $\left(\boldsymbol{d}_{\mathbf{3}}-\mathbf{1 t}\right)$ and $\mathrm{D}_{2} \mathrm{~N}-\ddot{\mathrm{C}}-\mathrm{OH}\left(\boldsymbol{d}_{\mathbf{2}}-\mathbf{1 t}\right)$. From the relative intensities of the IR bands we estimate a ratio of $5: 1$ between these two isotopologues. This finding can be explained by the acidity of the precursor oxalic acid monoamide: The $\mathrm{OD}$ deuterium can readily exchange while $\mathrm{ND}_{2}$ is rather stable. This also explains the absence of isotopologues like $\mathrm{H}_{2} \mathrm{~N}-\ddot{\mathrm{C}}-\mathrm{OD}$.

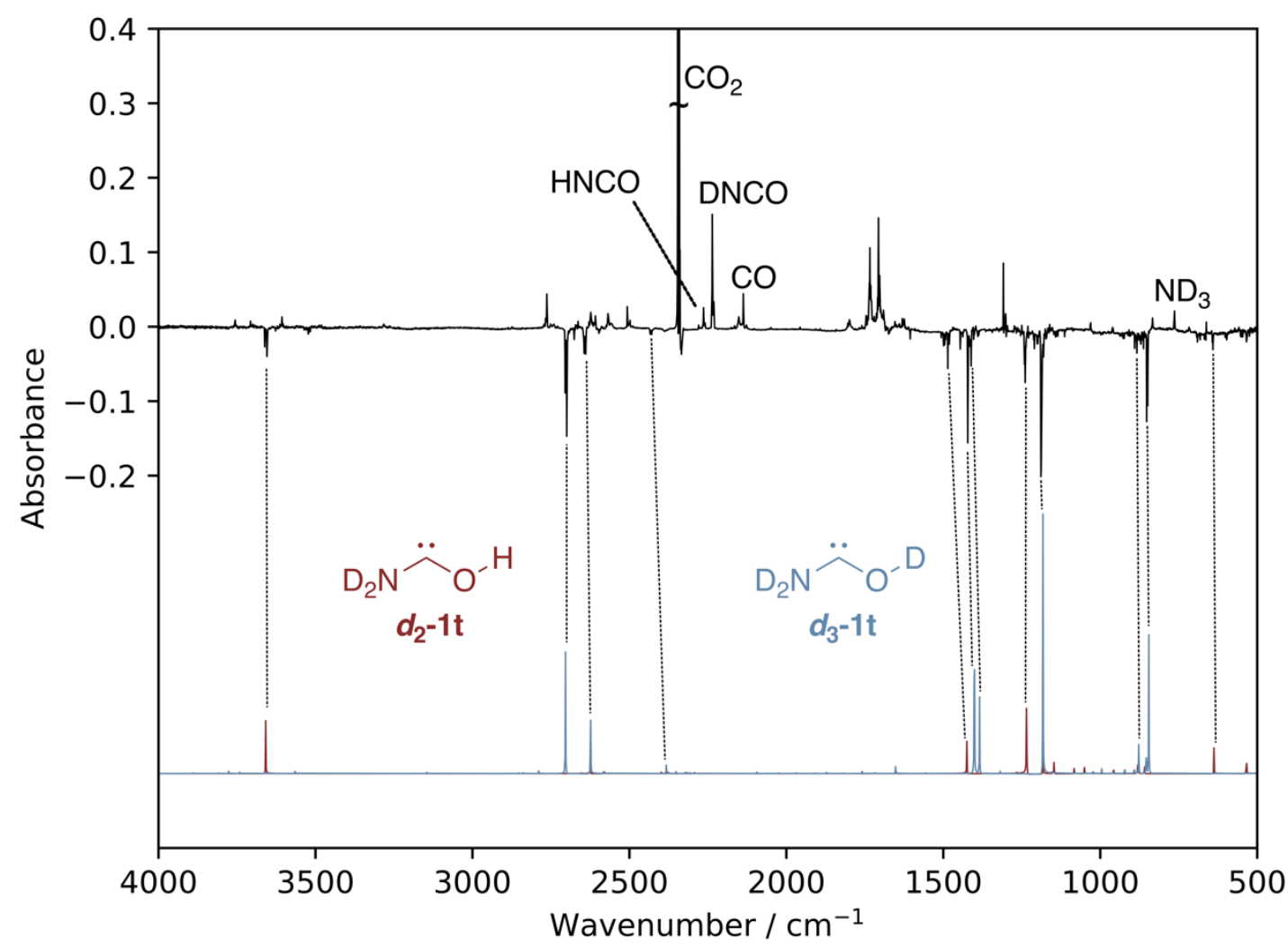

Figure S18: Experimental matrix IR difference spectrum of spectra measured before and after irradiation at $254 \mathrm{~nm}$ for $4 \mathrm{~min}$ of the pyrolysate of deuterated oxalic acid monoamide (black) and anharmonic spectra computed at the B3LYP/6-311++G(3df,3pd) level of theory of $\mathrm{D}_{2} \mathrm{~N}-$ $\ddot{\mathrm{C}}-\mathrm{OH}\left(\boldsymbol{d}_{\mathbf{2}}-\mathbf{1 t}\right.$; red) and $\mathrm{D}_{2} \mathrm{~N}-\ddot{C}-\mathrm{OD}\left(\boldsymbol{d}_{3}-\mathbf{1 t}\right.$; blue). The computed spectra are scaled $1: 5$ to simulate the observed ratio of the two isotopologues. Upon photolysis $\boldsymbol{d}_{\mathbf{3}}-\mathbf{1 t}$ reacts to $\mathrm{DNCO}+\mathrm{D} 2$ and CO + ND3 while HNCO results from photolysis of $\boldsymbol{d}_{\mathbf{2}}-\mathbf{1 t}$. 


\section{Matrix IR Assignments}

In Tables S1 to S8 we compare observed with computed matrix IR bands. The anharmonic computed values were used for band assignments. We provide the computed values of the harmonic vibrations as well as these are the basis for ZPVE corrections of electronic energies (vide infra) and comparison purposes. Generally, we find that anharmonic B3LYP IR spectra simulate experimental matrix spectra very reasonably.

Table S1: Comparison of experimental vibrational frequencies of transaminohydroxymethylene (1t) isolated in an $\mathrm{Ar}$ matrix at $3 \mathrm{~K}$ and computed vibrational frequencies at the B3LYP/6-311++G(3df,3pd) level of theory (unscaled). Only those overtones and combination bands are given, whose computed intensities exceed $10 \mathrm{~km} \mathrm{~mol}^{-1}$.

\begin{tabular}{|c|c|c|c|c|c|c|c|c|}
\hline$\#$ & Assignment ${ }^{\mathrm{a}}$ & Sym. & $\tilde{V}_{\text {anharm. }} / \mathrm{cm}^{-1}$ & $I_{\text {rel. }} / \mathrm{km} \mathrm{mol}^{-1}$ & $\tilde{v}_{\text {harm. }} / \mathrm{cm}^{-1}$ & $I_{\text {rel. }} / \mathrm{km} \mathrm{mol}^{-1}$ & $\tilde{v}_{\text {exp. }} / \mathrm{cm}^{-1}$ & $I_{\text {rel. }}{ }^{\mathrm{b}}$ \\
\hline $\mathrm{v}_{1}$ & $v(\mathrm{OH})$ & $a^{\prime}$ & 3658.8 & 117.2 & 3856.2 & 136.3 & $3661.8,3654.3$ & $\mathrm{~s}$ \\
\hline $\mathrm{v}_{2}$ & $v_{\text {as }}\left(\mathrm{NH}_{2}\right)$ & $a^{\prime}$ & 3517.0 & 60.9 & 3690.5 & 72.3 & $3537.0,3529.9$ & $\mathrm{~m}$ \\
\hline$v_{3}$ & $v_{\mathrm{s}}\left(\mathrm{NH}_{2}\right)$ & $a^{\prime}$ & 3266.6 & 7.2 & 3434.4 & 2.6 & n.o. & n.o. \\
\hline $\mathrm{v}_{4}$ & $\delta\left(\mathrm{NH}_{2}\right)$ & $a^{\prime}$ & 1603.0 & 72.2 & 1637.3 & 88.1 & $1608.0,1605.4$ & $\mathrm{~s}$ \\
\hline$V_{5}$ & $v(\mathrm{CN})+\delta(\mathrm{HOC})$ & $a^{\prime}$ & 1379.3 & 14.0 & 1425.4 & 19.8 & n.o. & n.o. \\
\hline $\mathrm{V}_{12}+\mathrm{V}_{10}$ & $\omega\left(\mathrm{NH}_{2}\right)+\tau\left(\mathrm{NH}_{2}\right)$ & $a^{\prime}$ & 1286.9 & 23.8 & 1315.4 & - & $1291.7,1279.7$ & w \\
\hline $\mathrm{V}_{6}$ & $v(\mathrm{CO})+\rho\left(\mathrm{NH}_{2}\right)$ & $a^{\prime}$ & 1247.1 & 144.8 & 1290.1 & 208.6 & $1250.6,1247.7$ & vs \\
\hline $2 x v_{11}$ & $2 \mathrm{x} \tau(\mathrm{OH})$ & $a^{\prime}$ & 1196.4 & 26.0 & 1257.2 & - & $1199.5,1191.8$ & vw \\
\hline $\mathrm{v}_{7}$ & $v(\mathrm{CN})+\delta(\mathrm{HOC})$ & $a^{\prime}$ & 1139.5 & 40.1 & 1182.1 & 41.9 & 1144.1 & vw \\
\hline $2 \times v_{12}$ & $2 x \omega\left(\mathrm{NH}_{2}\right)$ & $a^{\prime}$ & 1120.3 & 38.7 & 1104.3 & - & $1113.7,1110.6,1101.6$ & $w$ \\
\hline $\mathrm{V}_{8}$ & $v(\mathrm{CO})+\rho\left(\mathrm{NH}_{2}\right)$ & $a^{\prime}$ & 1010.4 & 108.7 & 1038.3 & 99.6 & $1012.9,1007.7$ & $\mathrm{~s}$ \\
\hline $\mathrm{V}_{10}$ & $\tau\left(\mathrm{NH}_{2}\right)$ & $a^{\prime \prime}$ & 736.8 & 1.8 & 763.2 & 2.8 & n.o. & n.o. \\
\hline $\mathrm{v}_{11}$ & $\tau(\mathrm{OH})$ & $a^{\prime \prime}$ & 603.3 & 184.1 & 628.6 & 143.0 & $602.5,598.2$ & vs \\
\hline$V_{9}$ & $\delta(\mathrm{OCN})$ & $a^{\prime}$ & 556.4 & 14.4 & 560.5 & 13.8 & 554.8. & w \\
\hline $\mathrm{V}_{12}$ & $\omega\left(\mathrm{NH}_{2}\right)$ & $a^{\prime \prime}$ & 551.9 & 78.4 & 552.2 & 129.5 & $551.5,545.0$ & $\mathrm{~s}$ \\
\hline
\end{tabular}

a: assignments ( $v=$ stretching, $\delta=$ bending, $\omega=$ wagging, $\tau=$ twisting, $\rho=$ rocking; s = symmetric, as $=$ antisymmetric).

b: rel. experimental intensities ( $v w=$ very weak, $\mathrm{w}=$ weak, $\mathrm{m}=$ middle, $\mathrm{s}=$ strong, $\mathrm{vs}=$ very strong; $\mathrm{n} . \mathrm{o} .=$ not observed). 
Table S2: Comparison of experimental vibrational frequencies of perdeuterated transaminohydroxymethylene $\left(\boldsymbol{d}_{\mathbf{3}}-\mathbf{1 t}\right)$ isolated in an Ar matrix at $3 \mathrm{~K}$ and computed vibrational frequencies at the B3LYP/6-311++G(3df,3pd) level of theory (unscaled). Only those overtones and combination bands are given, whose computed intensities exceed $10 \mathrm{~km} \mathrm{~mol}^{-1}$.

\begin{tabular}{|c|c|c|c|c|c|c|c|c|}
\hline$\#$ & Assignment $^{a}$ & Sym. & $\tilde{v}_{\text {anharm. }} / \mathrm{cm}^{-1}$ & $I_{\text {rel. }} / / \mathrm{km} \mathrm{mol}^{-1}$ & $\tilde{V}_{\text {harm. }} / \mathrm{cm}^{-1}$ & $I_{\text {rel. }} / \mathrm{km} \mathrm{mol}^{-1}$ & $\tilde{V}_{\text {exp. }} / \mathrm{cm}^{-1}$ & $I_{\text {rel. }}^{b}$ \\
\hline $\mathrm{V}_{1}$ & $v(\mathrm{OD})$ & $a^{\prime}$ & 2704.2 & 67.1 & 2808.6 & 75.0 & $2705.1 ; 2700.0$ & $\mathrm{~m}$ \\
\hline$v_{2}$ & $v_{\text {as }}\left(\mathrm{ND}_{2}\right)$ & $a^{\prime}$ & 2624.4 & 38.0 & 2720.6 & 43.5 & $2644.4,2642.8,2639.5$ & w \\
\hline $\mathrm{V}_{3}$ & $v_{\mathrm{s}}\left(\mathrm{ND}_{2}\right)$ & $a^{\prime}$ & 2383.1 & 4.9 & 2489.0 & 5.6 & $2434.0,2428.9$ & vw \\
\hline $\mathrm{V}_{4}$ & $v(\mathrm{CN})+\delta\left(\mathrm{ND}_{2}\right)$ & $a^{\prime}$ & 1402.2 & 122.0 & 1438.9 & 145.4 & 1422.5 & $\mathrm{~m}$ \\
\hline$v_{9}+v_{7}$ & $\delta(\mathrm{OCN})+\delta(\mathrm{DOC})$ & $a^{\prime}$ & 1385.4 & 14.8 & 1416.3 & - & 1411.4 & w \\
\hline$v_{5}$ & $v(\mathrm{CO})$ & $a^{\prime}$ & 1183.8 & 157.2 & 1212.8 & 158.0 & $1189.3,1188.2$ & s \\
\hline $\mathrm{v}_{6}$ & $\delta\left(\mathrm{ND}_{2}\right)$ & $a^{\prime}$ & 1120.1 & 0.3 & 1150.2 & 1.8 & n.o. & n.o. \\
\hline $\mathrm{v}_{7}$ & $\delta(\mathrm{DOC})$ & $a^{\prime}$ & 923.4 & 3.7 & 939.8 & 6.7 & n.o. & n.o. \\
\hline $2 \times v_{12}$ & $2 x \omega\left(N_{2}\right)$ & $a^{\prime}$ & 855.2 & 15.6 & 852.2 & - & n.o. & n.o. \\
\hline $\mathrm{v}_{8}$ & $\rho\left(\mathrm{ND}_{2}\right)+\delta(\mathrm{DOC})$ & $a^{\prime}$ & 846.5 & 76.1 & 867.9 & 80.1 & $852.5,848.8$ & $w$ \\
\hline $\mathrm{V}_{10}$ & $\tau\left(\mathrm{ND}_{2}\right)$ & $a^{\prime \prime}$ & 576.2 & 0.1 & 591.2 & 0.1 & n.o. & n.o. \\
\hline $\mathrm{V}_{9}$ & $\delta(\mathrm{OCN})$ & $a^{\prime}$ & 472.5 & 10.0 & 476.5 & 9.6 & n.o. & n.o. \\
\hline $\mathrm{v}_{11}$ & $\tau(\mathrm{OD})$ & $a^{\prime \prime}$ & 450.4 & 40.9 & 465.5 & 45.5 & $449.7,445.7$ & $w$ \\
\hline $\mathrm{v}_{12}$ & $\omega\left(\mathrm{ND}_{2}\right)$ & $a^{\prime \prime}$ & 425.0 & 99.6 & 426.1 & 98.8 & $420.0,418.9$ & $\mathrm{~m}$ \\
\hline
\end{tabular}

a: assignments ( $v=$ stretching, $\delta=$ bending, $\omega=$ wagging, $\tau=$ twisting, $\rho=$ rocking; s = symmetric, as $=$ antisymmetric).

b: rel. experimental intensities ( $v w=$ very weak, $\mathrm{w}=$ weak, $\mathrm{m}=$ middle, $\mathrm{s}=$ strong, $\mathrm{vs}=$ very strong; $\mathrm{n} . \mathrm{o} .=$ not observed).

Table S3: Comparison of experimental vibrational frequencies of dideuterated transaminohydroxymethylene $\left(\boldsymbol{d}_{\mathbf{2}}-\mathbf{- 1} \mathbf{t}, \mathrm{D}_{2} \mathrm{NCOH}\right)$ isolated in an $\mathrm{Ar}$ matrix at $3 \mathrm{~K}$ and computed vibrational frequencies at the B3LYP/6-311++G(3df,3pd) level of theory (unscaled). Only those overtones and combination bands are given, whose computed intensities exceed $10 \mathrm{~km} \mathrm{~mol}^{-}$ 1 .

\begin{tabular}{|c|c|c|c|c|c|c|c|c|}
\hline$\#$ & Assignment $^{\mathrm{a}}$ & Sym. & $\tilde{v}_{\text {anharm. }} / \mathrm{cm}^{-1}$ & $I_{\text {rel. }} / \mathrm{km} \mathrm{mol}^{-1}$ & $\tilde{v}_{\text {harm. }} / \mathrm{cm}^{-1}$ & $I_{\text {rel. }} / \mathrm{km} \mathrm{mol}^{-1}$ & $\tilde{v}_{\text {exp. }} / \mathrm{cm}^{-1}$ & $I_{\text {rel. }}{ }^{b}$ \\
\hline $\mathrm{v}_{1}$ & $v(\mathrm{OH})$ & $a^{\prime}$ & 3659.7 & 118.1 & 3856.1 & 137.9 & $3661.7 ; 3654.6$ & $\mathrm{~s}$ \\
\hline $\mathrm{v}_{2}$ & $v_{\text {as }}\left(\mathrm{ND}_{2}\right)$ & $a^{\prime}$ & 2625.7 & 31.9 & 2720.8 & 39.7 & n.o. & n.o. \\
\hline$v_{3}$ & $v_{\mathrm{s}}\left(\mathrm{ND}_{2}\right)$ & $a^{\prime}$ & 2377.5 & 3.0 & 2489.0 & 5.7 & n.o. & n.o. \\
\hline $\mathrm{V}_{4}$ & $v(\mathrm{CN})+\delta(\mathrm{HOC})$ & $a^{\prime}$ & 1426.1 & 81.4 & 1468.4 & 84.8 & 1446.1 & $\mathrm{~m}$ \\
\hline$v_{5}$ & $\delta(\mathrm{HOC})+v(\mathrm{CO})$ & $a^{\prime}$ & 1236.0 & 231.8 & 1269.0 & 227.0 & $1241.4 ; 1239.1$ & vs \\
\hline $2 x v_{11}$ & $2 \times \tau\left(N_{2}\right)$ & $a^{\prime}$ & 1085.3 & 12.6 & 1104.1 & - & n.o. & n.o. \\
\hline$v_{6}$ & $v(\mathrm{CO})$ & $a^{\prime}$ & 1184.2 & 46.0 & 1203.5 & 60.7 & n.o. & n.o. \\
\hline $\mathrm{V}_{7}$ & $\delta(\mathrm{HOC})$ & $a^{\prime}$ & 1052.4 & 14.3 & 1090.8 & 20.1 & n.o. & n.o. \\
\hline $\mathrm{V}_{8}$ & $\rho\left(\mathrm{ND}_{2}\right)+v(\mathrm{CO})$ & $a^{\prime}$ & 881.5 & 46.2 & 899.2 & 48.6 & 883.4 & w \\
\hline $2 \times v_{12}$ & $2 x \omega\left(N_{2}\right)$ & $a^{\prime}$ & 860.1 & 16.1 & 854.2 & - & $643.0 ; 641.1$ & w \\
\hline $\mathrm{v}_{10}$ & $\tau(\mathrm{OH})$ & $a^{\prime \prime}$ & 639.6 & 57.7 & 667.9 & 63.5 & n.o. & n.o. \\
\hline $\mathrm{v}_{11}$ & $\tau\left(\mathrm{ND}_{2}\right)$ & $a^{\prime \prime}$ & 536.2 & 49.6 & 552.0 & 43.8 & n.o. & n.o. \\
\hline $\mathrm{V}_{9}$ & $\delta(\mathrm{OCN})+\rho\left(\mathrm{ND}_{2}\right)$ & $a^{\prime}$ & 485.7 & 7.8 & 489.7 & 7.5 & n.o. & n.o. \\
\hline $\mathrm{V}_{12}$ & $\omega\left(\mathrm{ND}_{2}\right)$ & $a^{\prime \prime}$ & 426.5 & 76.2 & 427.1 & 80.9 & n.o. & n.o. \\
\hline
\end{tabular}

a: assignments $(v=$ stretching, $\delta=$ bending, $\omega=$ wagging, $\tau=$ twisting, $\rho=$ rocking; $\mathrm{s}=$ symmetric, as $=$ antisymmetric).

b: rel. experimental intensities ( $v w=$ very weak, $w=$ weak, $m=$ middle, $s=$ strong, $v s=$ very strong; $n . o .=$ not observed). 
Table S4: Computed vibrational frequencies of cis-aminohydroxymethylene (1c) at the B3LYP/6-311++G(3df,3pd) level of theory (unscaled). Only those overtones and combination bands are given, whose computed intensities exceed $10 \mathrm{~km} \mathrm{~mol}^{-1}$. We did not observe this species in an Ar matrix at $3 \mathrm{~K}$.

\begin{tabular}{|c|c|c|c|c|c|c|c|c|}
\hline$\#$ & Assignment $^{\mathrm{a}}$ & Sym. & $\tilde{V}_{\text {anharm. }} / \mathrm{cm}^{-1}$ & $I_{\text {rel. }} / \mathrm{km} \mathrm{mol}^{-1}$ & $\tilde{V}_{\text {harm. }} / \mathrm{cm}^{-1}$ & $I_{\text {rel. }} / \mathrm{km} \mathrm{mol}^{-1}$ & $\tilde{v}_{\text {exp. }} / \mathrm{cm}^{-1}$ & $I_{\text {rel. }}{ }^{b}$ \\
\hline $\mathrm{v}_{1}$ & $v_{\text {as }}\left(\mathrm{NH}_{2}\right)$ & $a^{\prime}$ & 3497.2 & 55.6 & 3668.4 & 66.2 & n.o. & n.o. \\
\hline $\mathrm{v}_{2}$ & $v(\mathrm{OH})$ & $a^{\prime}$ & 3213.4 & 81.5 & 3444.9 & 64.0 & n.o. & n.o. \\
\hline $\mathrm{V}_{3}$ & $v_{s}\left(\mathrm{NH}_{2}\right)$ & $a^{\prime}$ & 3114.6 & 48.0 & 3346.2 & 46.1 & n.o. & n.o. \\
\hline $\mathrm{V}_{4}$ & $\delta\left(\mathrm{NH}_{2}\right)$ & $a^{\prime}$ & 1597.3 & 55.2 & 1641.9 & 77.7 & n.o. & n.o. \\
\hline $\mathrm{V}_{5}$ & $v(\mathrm{CN})+\delta(\mathrm{HOC})$ & $a^{\prime}$ & 1350.3 & 199.3 & 1391.0 & 237.5 & n.o. & n.o. \\
\hline$v_{6}$ & $v(\mathrm{CO})+\rho\left(\mathrm{NH}_{2}\right)$ & $a^{\prime}$ & 1255.2 & 8.5 & 1325.6 & 24.9 & n.o. & n.o. \\
\hline $\mathrm{V}_{7}$ & $v(\mathrm{CN})+\delta(\mathrm{HOC})$ & $a^{\prime}$ & 1139.3 & 2.1 & 1160.3 & 25.2 & n.o. & n.o. \\
\hline $\mathrm{V}_{8}$ & $v(\mathrm{CO})+\rho\left(\mathrm{NH}_{2}\right)$ & $a^{\prime}$ & 1037.1 & 5.9 & 1063.2 & 26.0 & n.o. & n.o. \\
\hline $\mathrm{V}_{10}$ & $\tau\left(\mathrm{NH}_{2}\right)$ & $a^{\prime \prime}$ & 725.2 & 29.2 & 751.1 & 30.8 & n.o. & n.o. \\
\hline $\mathrm{v}_{11}$ & $\tau(\mathrm{OH})$ & $a^{\prime \prime}$ & 592.9 & 120.2 & 603.7 & 84.1 & n.o. & n.o. \\
\hline$V_{9}$ & $\delta(\mathrm{OCN})$ & $a^{\prime}$ & 584.4 & 2.1 & 586.8 & 2.7 & n.o. & n.o. \\
\hline $\mathrm{V}_{12}$ & $\omega\left(\mathrm{NH}_{2}\right)$ & $a^{\prime \prime}$ & 527.5 & 65.4 & 520.0 & 110.3 & n.o. & n.o. \\
\hline
\end{tabular}

a: assignments ( $v=$ stretching, $\delta=$ bending, $\omega=$ wagging, $\tau=$ twisting, $\rho=$ rocking; $\mathrm{s}=$ symmetric, as $=$ antisymmetric).

b: rel. experimental intensities ( $\mathrm{vw}=$ very weak, $\mathrm{w}=$ weak, $\mathrm{m}=$ middle, $\mathrm{s}=$ strong, $\mathrm{vs}=$ very strong; $\mathrm{n} . \mathrm{o}$. = not observed). 
Table S5: Comparison of experimental vibrational frequencies of cis-oxalic acid monoamide (2c) isolated in an Ar matrix at $3 \mathrm{~K}$ and computed vibrational frequencies at the B3LYP/6$311++G(3 d f, 3 p d)$ level of theory (unscaled). Only those overtones and combination bands are given, whose computed intensities exceed $10 \mathrm{~km} \mathrm{~mol}^{-1}$.

\begin{tabular}{|c|c|c|c|c|c|c|c|c|}
\hline$\#$ & Assignment $^{a}$ & Sym. & $\tilde{V}_{\text {Vanharm. }} / \mathrm{cm}^{-1}$ & $I_{\text {rel. }} / \mathrm{km} \mathrm{mol}^{-1}$ & $\tilde{V}_{\text {harm. }} / \mathrm{cm}^{-1}$ & $I_{\text {rel. }} / \mathrm{km} \mathrm{mol}^{-1}$ & $\tilde{v}_{\text {exp. }} / \mathrm{cm}^{-1}$ & $I_{\text {rel. }}{ }^{b}$ \\
\hline $\mathrm{v}_{1}$ & $v_{\text {as }}\left(\mathrm{NH}_{2}\right)$ & $a^{\prime}$ & 3527.3 & 75.0 & 3708.4 & 86.9 & $3540.8,3537.1$ & $\mathrm{~m}$ \\
\hline $\mathrm{v}_{2}$ & $v(\mathrm{OH})$ & $a^{\prime}$ & 3417.2 & 59.7 & 3575.4 & 60.4 & 3417.9 & $\mathrm{~m}$ \\
\hline $\mathrm{V}_{3}$ & $v_{s}\left(\mathrm{NH}_{2}\right)$ & $a^{\prime}$ & 3384.8 & 117.5 & 3596.0 & 146.4 & 3371.3 & $\mathrm{~m}$ \\
\hline $\mathrm{V}_{4}$ & $v(\mathrm{C}=0)$ & $a^{\prime}$ & 1818.5 & 232.3 & 1847.5 & 276.4 & 1816.1 & $\mathrm{~m}$ \\
\hline $\mathrm{v}_{18}+\mathrm{v}_{9}$ & $\tau\left(\mathrm{NH}_{2}\right)+\delta(\mathrm{HOC})$ & $a^{\prime \prime}$ & 1783.4 & 33.9 & 1838.6 & - & 1800.0 & w \\
\hline$V_{5}$ & $v(\mathrm{C}=0)$ & $a^{\prime}$ & 1740.7 & 282.3 & 1772.4 & 340.2 & 1737.8 & vs \\
\hline$v_{13}+v_{9}$ & $\delta(\mathrm{O}=\mathrm{C}-\mathrm{C})+\delta(\mathrm{HOC})$ & $a^{\prime}$ & 1692.6 & 16.3 & 1746.7 & - & 1687.3 & vw \\
\hline$v_{6}$ & $\delta\left(\mathrm{NH}_{2}\right)$ & $a^{\prime}$ & 1571.3 & 39.8 & 1603.8 & 75.0 & 1569.4 & $\mathrm{~m}$ \\
\hline $2 \times v_{11}$ & $2 \times v(C C)$ & $a^{\prime}$ & 1559.8 & 15.3 & 1598.8 & - & 1528.0 & vw \\
\hline $\mathrm{V}_{7}$ & $v(\mathrm{CC})$ & $a^{\prime}$ & 1393.7 & 35.6 & 1428.1 & 151.2 & 1421.7 & $\mathrm{~m}$ \\
\hline $2 \times v_{17}$ & $2 \times \delta(\mathrm{OH})$ & $a^{\prime}$ & 1373.2 & 41.0 & 1498.5 & - & 1383.0 & $\mathrm{vw}$ \\
\hline $\mathrm{v}_{13}+\mathrm{v}_{11}$ & $\begin{array}{c}\delta(\mathrm{O}=\mathrm{C}-\mathrm{C})+ \\
v(\mathrm{CC})\end{array}$ & $a^{\prime}$ & 1311.7 & 58.0 & 1341.6 & - & 1315.2 & vw \\
\hline $\mathrm{v}_{8}$ & $v(\mathrm{CN})$ & $a^{\prime}$ & 1292.4 & 398.1 & 1337.1 & 387.3 & 1301.8 & vs \\
\hline $2 \times v_{12}$ & $2 \times \delta\left(\mathrm{O}=\mathrm{C}-\mathrm{NH}_{2}\right)$ & $a^{\prime}$ & 1239.2 & 14.6 & 1311.8 & - & 1258.8 & w \\
\hline $\mathrm{v}_{19}+\mathrm{v}_{17}$ & $\begin{array}{c}\omega\left(\mathrm{H}_{2} \mathrm{~N}-\mathrm{C}=\mathrm{O}\right)+ \\
\delta(\mathrm{OH})\end{array}$ & $a^{\prime}$ & 1184.1 & 15.5 & 1222.3 & - & 1228.5 & $\mathrm{vw}$ \\
\hline $\mathrm{V}_{9}$ & $\delta(\mathrm{HOC})$ & $a^{\prime}$ & 1156.5 & 0.2 & 1204.6 & 13.0 & 1181.9 & w \\
\hline $\mathrm{v}_{10}$ & $\rho\left(\mathrm{NH}_{2}\right)$ & $a^{\prime}$ & 1133.8 & 2.6 & 1101.8 & 1.8 & n.o. & n.o. \\
\hline $\mathrm{v}_{14}+\mathrm{v}_{19}$ & $\begin{array}{c}\delta\left(\mathrm{H}_{2} \mathrm{~N}-\mathrm{C}=\mathrm{O}\right)+ \\
\omega\left(\mathrm{H}_{2} \mathrm{~N}-\mathrm{C}=\mathrm{O}\right)\end{array}$ & $a^{\prime \prime}$ & 950.3 & 17.7 & 864.9 & - & 936.0 & w \\
\hline $2 \times v_{14}$ & $2 \times \delta\left(\mathrm{H}_{2} \mathrm{~N}-\mathrm{C}=\mathrm{O}\right)$ & $a^{\prime}$ & 907.8 & 30.2 & 783.6 & - & 892.7 & $\mathrm{~m}$ \\
\hline $\mathrm{v}_{16}$ & $\tau(\mathrm{CC})$ & $a^{\prime \prime}$ & 829.1 & 1.2 & 836.8 & 0.0 & 820.6 & w \\
\hline $\mathrm{v}_{11}$ & $v(\mathrm{CC})$ & $a^{\prime}$ & 778.8 & 8.7 & 799.4 & 9.6 & 793.3 & w \\
\hline $\mathrm{v}_{17}$ & $\delta(\mathrm{OH})$ & $a^{\prime \prime}$ & 699.4 & 83.2 & 749.3 & 93.6 & 703.2 & $\mathrm{~m}$ \\
\hline $\mathrm{v}_{18}$ & $\tau\left(\mathrm{NH}_{2}\right)$ & $a^{\prime \prime}$ & 628.6 & 11.1 & 634.0 & 13.8 & 628.2 & $w$ \\
\hline $\mathrm{v}_{12}$ & $\delta\left(\mathrm{O}=\mathrm{C}-\mathrm{NH}_{2}\right)$ & $a^{\prime}$ & 626.2 & 3.7 & 655.9 & 3.7 & 655.6 & $\mathrm{vw}$ \\
\hline $\mathrm{v}_{13}$ & $\delta(\mathrm{O}=\mathrm{C}-\mathrm{C})$ & $a^{\prime}$ & 533.8 & 1.7 & 542.2 & 2.2 & n.o. & n.o. \\
\hline $\mathrm{V}_{19}$ & $\omega\left(\mathrm{H}_{2} \mathrm{~N}-\mathrm{C}=\mathrm{O}\right)$ & $a^{\prime \prime}$ & 485.1 & 64.7 & 473.1 & 120.3 & n.o. & n.o. \\
\hline $\mathrm{v}_{14}$ & $\delta\left(\mathrm{H}_{2} \mathrm{~N}-\mathrm{C}=\mathrm{O}\right)$ & $a^{\prime}$ & 431.0 & 125.2 & 391.8 & 98.5 & 436.5 & s \\
\hline $\mathrm{v}_{20}$ & $\omega\left(\mathrm{NH}_{2}\right)$ & $a^{\prime \prime}$ & 395.4 & 6.4 & 402.6 & 6.5 & 406.5 & $\mathrm{~m}$ \\
\hline $\mathrm{v}_{15}$ & $\rho\left(\mathrm{H}_{2} \mathrm{~N}-\mathrm{C}=\mathrm{O}\right)$ & $a^{\prime}$ & 264.3 & 42.4 & 267.8 & 41.9 & o.o.r. & o.o.r. \\
\hline $\mathrm{v}_{21}$ & $\tau\left(\mathrm{H}_{2} \mathrm{~N}-\mathrm{C}=\mathrm{O}\right)$ & $a^{\prime \prime}$ & 111.9 & 10.3 & 113.4 & 8.0 & o.o.r. & o.o.r. \\
\hline
\end{tabular}

a: assignments ( $v=$ stretching, $\delta=$ bending, $\omega=$ wagging, $\tau=$ twisting, $\rho=$ rocking; $\mathrm{s}=$ symmetric, as $=$ antisymmetric).

b: rel. experimental intensities ( $v w=$ very weak, $w=$ weak, $m=$ middle, $s=$ strong, vs = very strong; $n . o .=$ not observed, o.o.r $=$ out of range). 
Table S6: Comparison of experimental vibrational frequencies of perdeuterated cis-oxalic acid monoamide $\left(\boldsymbol{d}_{\mathbf{3}}-\mathbf{2 c}\right)$ isolated in an Ar matrix at $3 \mathrm{~K}$ and computed vibrational frequencies at the $B 3 L Y P / 6-311++G(3 d f, 3 p d)$ level of theory (unscaled). Only those overtones and combination bands are given, whose computed intensities exceed $10 \mathrm{~km} \mathrm{~mol}^{-1}$.

\begin{tabular}{|c|c|c|c|c|c|c|c|c|}
\hline$\#$ & Assignment & Sym. & $\tilde{V}_{\text {anharm. }} / \mathrm{cm}^{-1}$ & $I_{\text {rel. }} / \mathrm{km} \mathrm{mol}^{-1}$ & $\tilde{V}_{\text {harm. }} / \mathrm{cm}^{-1}$ & $I_{\text {rel. }} / \mathrm{km} \mathrm{mol}^{-1}$ & $\tilde{v}_{\text {exp. }} / \mathrm{cm}^{-1}$ & $I_{\text {rel. }}$ \\
\hline $\mathrm{v}_{1}$ & $v_{\text {as }}\left(N_{2}\right)$ & $a^{\prime}$ & 2648.9 & 41.5 & 2749.3 & 49.8 & 2660.6 & $\mathrm{~m}$ \\
\hline$v_{8}+v_{6}$ & $\delta\left(\mathrm{ND}_{2}\right)+v_{\text {as }}(\mathrm{CCN})$ & $a^{\prime}$ & 2521.6 & 10.5 & 2565.7 & - & 2561.6 & w \\
\hline $\mathrm{V}_{2}$ & $v(\mathrm{OD})$ & $a^{\prime}$ & 2511.3 & 68.3 & 2616.4 & 80.6 & 2502.2 & $\mathrm{~m}$ \\
\hline $\mathrm{V}_{3}$ & $v_{\mathrm{s}}\left(\mathrm{ND}_{2}\right)$ & $a^{\prime}$ & 2476.2 & 31.7 & 2581.1 & 54.7 & 2489.2 & s \\
\hline $\mathrm{V}_{12}+\mathrm{V}_{7}$ & $\delta(\mathrm{OCN})+v_{\mathrm{s}}(\mathrm{CCN})$ & $a^{\prime}$ & 1831.3 & 25.6 & 1870.9 & - & n.o. & n.o. \\
\hline $\mathrm{v}_{4}$ & $v(\mathrm{CO})$ & $a^{\prime}$ & 1805.4 & 267.7 & 1838.5 & 301.7 & 1799.4 & s \\
\hline$v_{5}$ & $v(\mathrm{CO})$ & $a^{\prime}$ & 1731.4 & 282.4 & 1763.6 & 338.0 & 1728.9 & vs \\
\hline $\mathrm{v}_{11}+\mathrm{v}_{9}$ & $v(\mathrm{CC})+\delta(\mathrm{OD})$ & $a^{\prime}$ & 1705.4 & 16.0 & 1754.6 & - & n.o. & n.o. \\
\hline$v_{6}$ & $v_{\text {as }}(\mathrm{CCN})$ & $a^{\prime}$ & 1397.6 & 18.8 & 1433.4 & 18.1 & 1426.9 & w \\
\hline$V_{7}$ & $\nu_{\mathrm{s}}(\mathrm{CCN})$ & $a^{\prime}$ & 1263.3 & 196.0 & 1287.3 & 370.4 & 1270.4 & $\mathrm{~s}$ \\
\hline $\mathrm{V}_{13}+\mathrm{V}_{11}$ & $\delta_{\mathrm{s}}(\mathrm{CC})+v(\mathrm{CC})$ & $a^{\prime}$ & 1245.4 & 150.2 & 1279.0 & - & 1263.0 & s \\
\hline $\mathrm{V}_{19}+\mathrm{V}_{16}$ & $\tau\left(\mathrm{ND}_{2}\right)+\delta_{\text {as }}(\mathrm{CC})$ & $a^{\prime}$ & 1227.6 & 12.5 & 1245.2 & - & 1217.0 & w \\
\hline $\mathrm{V}_{8}$ & $\delta\left(\mathrm{ND}_{2}\right)$ & $a^{\prime}$ & 1106.1 & 7.1 & 1132.3 & 11.2 & 1109.3 & w \\
\hline$V_{9}$ & $\delta(\mathrm{OD})$ & $a^{\prime}$ & 963.7 & 28.7 & 994.7 & 36.9 & 968.5 & $\mathrm{~m}$ \\
\hline $\mathrm{V}_{10}$ & $\delta(\mathrm{CND})$ & $a^{\prime}$ & 932.6 & 16.5 & 941.4 & 18.0 & 929.8 & $\mathrm{~m}$ \\
\hline $\mathrm{V}_{16}$ & $\delta_{\text {as }}(\mathrm{CC})$ & $a^{\prime \prime}$ & 824.4 & 0.1 & 833.9 & 0.3 & n.o. & n.o. \\
\hline $\mathrm{v}_{11}$ & $v(\mathrm{CC})$ & $a^{\prime}$ & 744.6 & 7.0 & 759.9 & 9.7 & 756.7 & vw \\
\hline $2 x v_{20}$ & $2 x \omega\left(N D_{2}\right)$ & $a^{\prime}$ & 692.7 & 16.8 & 601.9 & - & 703.5 & vw \\
\hline $\mathrm{V}_{12}$ & $\delta(\mathrm{OCN})$ & $a^{\prime}$ & 576.7 & 9.0 & 583.6 & 10.7 & 576.9 & w \\
\hline $\mathrm{V}_{17}$ & $\delta(\mathrm{OD})$ & $a^{\prime \prime}$ & 526.0 & 80.8 & 547.9 & 51.7 & 522.3 & $\mathrm{~s}$ \\
\hline $\mathrm{v}_{13}$ & $\delta_{\mathrm{s}}(\mathrm{CC})$ & $a^{\prime}$ & 518.0 & 2.4 & 519.1 & 3.2 & 518.0 & w \\
\hline $\mathrm{V}_{18}$ & $\delta(\mathrm{OCC})$ & $a^{\prime \prime}$ & 515.3 & 0.5 & 531.4 & 26.9 & 512.0 & vw \\
\hline $\mathrm{V}_{19}$ & $\tau\left(\mathrm{ND}_{2}\right)$ & $a^{\prime \prime}$ & 405.1 & 41.5 & 411.2 & 34.9 & 398.1 & s \\
\hline $\mathrm{V}_{14}$ & $\rho\left(\mathrm{ND}_{2}\right)$ & $a^{\prime}$ & 366.8 & 9.3 & 373.3 & 9.8 & n.o. & n.o. \\
\hline $\mathrm{V}_{20}$ & $\omega\left(N_{2}\right)$ & $a^{\prime \prime}$ & 329.2 & 48.8 & 301.0 & 67.4 & o.o.r. & o.o.r. \\
\hline $\mathrm{V}_{15}$ & $\rho(\mathrm{OCN})$ & $a^{\prime}$ & 246.0 & 35.5 & 248.8 & 35.0 & o.o.r. & o.o.r. \\
\hline$v_{21}$ & $\omega(\mathrm{OCN})$ & $a^{\prime \prime}$ & 108.9 & 9.8 & 111.0 & 8.4 & o.o.r. & o.o.r. \\
\hline
\end{tabular}

a: assignments $(v=$ stretching, $\delta=$ bending, $\omega=$ wagging, $\tau=$ twisting, $\rho=$ rocking; $\mathrm{s}=$ symmetric, as $=$ antisymmetric).

b: rel. experimental intensities ( $v w=$ very weak, $w=$ weak, $m=$ middle, $s=$ strong, $v s=$ very strong; .o. = not observed, o.o.r = out of range). 
Table S7: Comparison of experimental vibrational frequencies of trans-oxalic acid monoamide (2t) isolated in an Ar matrix at $3 \mathrm{~K}$ and computed vibrational frequencies at the B3LYP/6$311++G(3 d f, 3 p d)$ level of theory (unscaled). Only those overtones and combination bands are given, whose computed intensities exceed $10 \mathrm{~km} \mathrm{~mol}^{-1}$.

\begin{tabular}{|c|c|c|c|c|c|c|c|c|}
\hline$\#$ & Assignment $^{\mathrm{a}}$ & Sym. & $\tilde{v}_{\text {anhharm. }} / \mathrm{cm}^{-1}$ & $I_{\text {rel. }} / \mathrm{km} \mathrm{mol}^{-1}$ & $\tilde{V}_{\text {harm. }} / \mathrm{cm}^{-1}$ & $I_{\text {rel. }} / \mathrm{km} \mathrm{mol}^{-1}$ & $\tilde{v}_{\text {exp. }} / \mathrm{cm}^{-1}$ & $I_{\text {rel. }}{ }^{b}$ \\
\hline $\mathrm{v}_{1}$ & $v(\mathrm{OH})$ & $a^{\prime}$ & 3564.7 & 71.8 & 3749.3 & 84.3 & 3555.1 & s \\
\hline $\mathrm{v}_{2}$ & $v_{\text {as }}\left(\mathrm{NH}_{2}\right)$ & $a^{\prime}$ & 3535.6 & 66.9 & 3719.0 & 79.7 & 3546.4 & $\mathrm{~m}$ \\
\hline$v_{3}$ & $v_{\mathrm{s}}\left(\mathrm{NH}_{2}\right)$ & $a^{\prime}$ & 3421.8 & 42.6 & 3584.5 & 56.3 & 3428.3 & w \\
\hline $\mathrm{V}_{4}$ & $v_{s}(\mathrm{C}=0)$ & $a^{\prime}$ & 1769.9 & 4.0 & 1803.6 & 3.7 & 1769.1 & $\mathrm{vw}$ \\
\hline $\mathrm{V}_{5}$ & $v_{\text {as }}(\mathrm{C}=\mathrm{O})$ & $a^{\prime}$ & 1764.5 & 587.9 & 1793.5 & 646.1 & 1751.4 & vs \\
\hline $\mathrm{v}_{10}+\mathrm{v}_{7}$ & $\delta(\mathrm{O}=\mathrm{C}-\mathrm{C})+\rho\left(\mathrm{NH}_{2}\right)$ & $a^{\prime}$ & 1613.7 & 20.8 & 1620.5 & - & n.o. & n.o. \\
\hline$v_{6}$ & $\delta\left(\mathrm{NH}_{2}\right)$ & $a^{\prime}$ & 1562.0 & 88.3 & 1599.5 & 115.0 & 1566.4 & $\mathrm{~m}$ \\
\hline $\mathrm{V}_{7}$ & $\delta(\mathrm{O}=\mathrm{C}-\mathrm{C})$ & $a^{\prime}$ & 1375.3 & 1.9 & 1409.2 & 9.3 & 1398.3 & vw \\
\hline $\mathrm{v}_{8}$ & $\delta\left(\mathrm{H}_{2} \mathrm{~N}-\mathrm{C}-\mathrm{C}\right)$ & $a^{\prime}$ & 1296.6 & 30.6 & 1313.2 & 67.5 & n.o. & n.o. \\
\hline $2 \times v_{17}$ & $2 x \omega(C C)$ & $a^{\prime}$ & 1260.2 & 33.8 & 1334.1 & - & n.o. & n.o. \\
\hline $\mathrm{v}_{12}+\mathrm{v}_{17}$ & $\delta\left(\mathrm{O}=\mathrm{C}-\mathrm{NH}_{2}\right)+\omega(\mathrm{CC})$ & $a^{\prime \prime}$ & 1208.5 & 28.7 & 1289.2 & - & 1200.0 & v.w. \\
\hline $\mathrm{v}_{20}+\mathrm{v}_{11}$ & $\omega\left(\mathrm{NH}_{2}\right)+v(\mathrm{CC})$ & $a^{\prime \prime}$ & 1158.1 & 23.7 & 1184.5 & - & 1170.0 & vw \\
\hline$V_{9}$ & $v(\mathrm{C}-\mathrm{OH})$ & $a^{\prime}$ & 1143.6 & 198.6 & 1179.1 & 290.9 & 1144.2 & $\mathrm{~m}$ \\
\hline $\mathrm{v}_{10}$ & $\rho\left(\mathrm{NH}_{2}\right)$ & $a^{\prime}$ & 1104.2 & 36.1 & 1095.0 & 4.0 & n.o. & n.o. \\
\hline $\mathrm{v}_{14}+\mathrm{v}_{12}$ & $\begin{array}{c}\delta\left(\mathrm{H}_{2} \mathrm{~N}-\mathrm{C}=\mathrm{O}\right)+ \\
\delta\left(\mathrm{O}=\mathrm{C}-\mathrm{NH}_{2}\right)\end{array}$ & $a^{\prime}$ & 1001.7 & 11.5 & 965.0 & - & n.o. & n.o. \\
\hline $2 \times v_{14}$ & $2 \times \delta\left(\mathrm{H}_{2} \mathrm{~N}-\mathrm{C}=\mathrm{O}\right)$ & $a^{\prime}$ & 974.5 & 67.2 & 685.8 & - & 981.8 & $\mathrm{vw}$ \\
\hline $\mathrm{v}_{16}$ & $\tau(\mathrm{CC})$ & $a^{\prime \prime}$ & 826.1 & 2.1 & 835.4 & 5.1 & 826.9 & $\mathrm{vw}$ \\
\hline $\mathrm{v}_{11}$ & $v(\mathrm{CC})$ & $a^{\prime}$ & 754.3 & 8.7 & 772.3 & 7.9 & 760.0 & vw \\
\hline $\mathrm{V}_{17}$ & $\omega(\mathrm{CC})$ & $a^{\prime \prime}$ & 668.6 & 39.4 & 667.1 & 115.0 & n.o. & n.o. \\
\hline $\mathrm{V}_{28}$ & $\tau\left(\mathrm{NH}_{2}\right)$ & $a^{\prime \prime}$ & 610.7 & 65.5 & 615.5 & 71.7 & 607.5 & $\mathrm{~m}$ \\
\hline $\mathrm{v}_{12}$ & $\delta\left(\mathrm{O}=\mathrm{C}-\mathrm{NH}_{2}\right)$ & $a^{\prime}$ & 562.9 & 89.1 & 622.1 & 28.1 & 589.2 & w \\
\hline $\mathrm{v}_{13}$ & $\delta(\mathrm{O}=\mathrm{C}-\mathrm{C})$ & $a^{\prime}$ & 533.9 & 0.0 & 525.5 & 0.3 & n.o. & n.o. \\
\hline $\mathrm{V}_{19}$ & $\omega\left(\mathrm{H}_{2} \mathrm{~N}-\mathrm{C}=\mathrm{O}\right)$ & $a^{\prime \prime}$ & 443.2 & 98.5 & 436.2 & 12.5 & n.o. & n.o. \\
\hline $\mathrm{v}_{14}$ & $\delta\left(\mathrm{H}_{2} \mathrm{~N}-\mathrm{C}=\mathrm{O}\right)$ & $a^{\prime}$ & 432.6 & 48.5 & 342.9 & 178.5 & n.o. & n.o. \\
\hline $\mathrm{V}_{20}$ & $\omega\left(\mathrm{NH}_{2}\right)$ & $a^{\prime \prime}$ & 407.4 & 3.3 & 412.2 & 3.8 & n.o. & n.o. \\
\hline $\mathrm{V}_{15}$ & $\rho\left(\mathrm{H}_{2} \mathrm{~N}-\mathrm{C}=\mathrm{O}\right)$ & $a^{\prime}$ & 266.9 & 15.1 & 268.3 & 15.7 & o.o.r. & o.o.r. \\
\hline$v_{21}$ & $\tau\left(\mathrm{H}_{2} \mathrm{~N}-\mathrm{C}=\mathrm{O}\right)$ & $a^{\prime \prime}$ & 71.0 & 12.0 & 60.5 & 5.2 & o.o.r. & o.o.r. \\
\hline
\end{tabular}

a: assignments $(v=$ stretching, $\delta=$ bending, $\omega=$ wagging, $\tau=$ twisting, $\rho=$ rocking; $\mathrm{s}=$ symmetric, as $=$ antisymmetric).

b: rel. experimental intensities ( $v w=$ very weak, $w=$ weak, $m=$ middle, $s=$ strong, $v s=$ very strong; $n .0 .=$ not observed, o.o. $r=$ out of range). 
Table S8: Comparison of experimental vibrational frequencies of perdeuterated trans-oxalic acid monoamide $\left(\boldsymbol{d}_{\mathbf{3}}-\mathbf{2 t}\right)$ isolated in an Ar matrix at $3 \mathrm{~K}$ and computed vibrational frequencies at the B3LYP/6-311++G(3df,3pd) level of theory (unscaled). Only those overtones and combination bands are given, whose computed intensities exceed $10 \mathrm{~km} \mathrm{~mol}^{-1}$.

\begin{tabular}{|c|c|c|c|c|c|c|c|c|}
\hline$\#$ & Assignment $^{\mathrm{a}}$ & Sym. & $\tilde{v}_{\text {anharm. }} / \mathrm{cm}^{-1}$ & $I_{\text {rel. }} / \mathrm{km} \mathrm{mol}^{-1}$ & $\tilde{V}_{\text {harm. }} / \mathrm{cm}^{-1}$ & $I_{\text {rel. }} / \mathrm{km} \mathrm{mol}^{-1}$ & $\tilde{v}_{\text {exp. }} / \mathrm{cm}^{-1}$ & $I_{\text {rel. }}{ }^{b}$ \\
\hline $\mathrm{v}_{10}+\mathrm{v}_{4}$ & $\delta(\mathrm{CND})+v_{\mathrm{s}}(\mathrm{CO})$ & $a^{\prime}$ & 2682.7 & 13.8 & 2729.6 & - & n.o. & n.o. \\
\hline$v_{10}+v_{5}$ & $\delta(\mathrm{CND})+v_{\text {as }}(\mathrm{CO})$ & $a^{\prime}$ & 2680.4 & 15.5 & 2725.1 & - & n.o. & n.o. \\
\hline $\mathrm{v}_{1}$ & $\nu_{\text {as }}\left(\mathrm{ND}_{2}\right)$ & $a^{\prime}$ & 2655.3 & 39.0 & 2757.2 & 47.0 & 2623.3 & w \\
\hline $\mathrm{V}_{2}$ & $v(\mathrm{OD})$ & $a^{\prime}$ & 2628.5 & 23.9 & 2727.4 & 51.4 & 2600.9 & w \\
\hline $\mathrm{V}_{3}$ & $v_{\mathrm{s}}\left(\mathrm{ND}_{2}\right)$ & $a^{\prime}$ & 2493.4 & 26.5 & 2587.2 & 51.4 & 2531.3 & $\mathrm{~m}$ \\
\hline $\mathrm{v}_{12}+\mathrm{v}_{7}$ & $\delta(\mathrm{OCC})+v_{\mathrm{s}}(\mathrm{CCN})$ & $a^{\prime}$ & 1811.7 & 13.3 & 1848.0 & - & n.o. & n.o. \\
\hline$v_{13}+v_{7}$ & $\delta(\mathrm{OCC})+v_{\mathrm{s}}(\mathrm{CCN})$ & $a^{\prime}$ & 1764.2 & 83.3 & 1799.8 & - & 1759.8 & $\mathrm{~m}$ \\
\hline $\mathrm{v}_{4}$ & $v_{\mathrm{s}}(\mathrm{CO})$ & $a^{\prime}$ & 1758.6 & 6.5 & 1794.3 & 20.8 & n.o. & n.o. \\
\hline$v_{5}$ & $v_{\text {as }}(\mathrm{CO})$ & $a^{\prime}$ & 1758.4 & 512.2 & 1789.8 & 607.0 & 1746.5 & s \\
\hline $\mathrm{v}_{14}+\mathrm{v}_{6}$ & $\delta(\mathrm{OCN})+v_{\text {as }}(\mathrm{CCN})$ & $a^{\prime}$ & 1755.0 & 94.0 & 1795.7 & - & 1709.8 & $\mathrm{~m}$ \\
\hline$v_{6}$ & $v_{\text {as }}(\mathrm{CCN})$ & $a^{\prime}$ & 1375.9 & 19.1 & 1410.4 & 14.5 & 1309.2 & $\mathrm{~m}$ \\
\hline $\mathrm{v}_{7}$ & $v_{\mathrm{s}}(\mathrm{CCN})$ & $a^{\prime}$ & 1261.9 & 232.9 & 1291.6 & 313.1 & 1274.1 & s \\
\hline $\mathrm{v}_{18}+\mathrm{v}_{16}$ & $\tau\left(\mathrm{ND}_{2}\right)+\delta_{\text {as }}(\mathrm{CC})$ & $a^{\prime}$ & 1258.2 & 72.6 & 1283.8 & - & n.o. & n.o. \\
\hline $\mathrm{v}_{8}$ & $\delta\left(\mathrm{ND}_{2}\right)$ & $a^{\prime}$ & 1097.7 & 15.2 & 1119.5 & 19.8 & n.o. & n.o. \\
\hline $2 \times v_{13}$ & $\delta(\mathrm{OCC})+\delta(\mathrm{OCC})$ & $a^{\prime}$ & 1002.5 & 22.9 & 1016.5 & - & n.o. & n.o. \\
\hline$v_{9}$ & $\delta_{\mathrm{s}}(\mathrm{DOC})$ & $a^{\prime}$ & 998.6 & 28.2 & 1019.5 & 57.5 & 1003.4 & w \\
\hline $\mathrm{v}_{10}$ & $\delta(\mathrm{CND})$ & $a^{\prime}$ & 925.5 & 1.0 & 935.3 & 3.3 & n.o. & n.o. \\
\hline $\mathrm{v}_{16}$ & $\delta_{\text {as }}(\mathrm{CC})$ & $a^{\prime \prime}$ & 825.4 & 0.6 & 832.2 & 1.2 & n.o. & n.o. \\
\hline $2 \times v_{20}$ & $2 x \omega\left(N_{2}\right)$ & $a^{\prime}$ & 696.0 & 23.3 & 518.0 & - & n.o. & n.o. \\
\hline $\mathrm{v}_{11}$ & $v(\mathrm{CC})$ & $a^{\prime}$ & 665.3 & 12.6 & 684.1 & 10.5 & n.o. & n.o. \\
\hline $\mathrm{v}_{21}+\mathrm{v}_{13}$ & $\tau(\mathrm{OCN})+\delta(\mathrm{OCC})$ & $a^{\prime \prime}$ & 577.9 & 18.9 & 567.8 & - & n.o. & n.o. \\
\hline $\mathrm{V}_{12}$ & $\delta(\mathrm{OCC})$ & $a^{\prime}$ & 550.3 & 64.1 & 556.4 & 68.4 & 547.9 & $\mathrm{vw}$ \\
\hline $\mathrm{V}_{17}$ & $\delta_{\mathrm{s}}(\mathrm{CC})$ & $a^{\prime \prime}$ & 546.8 & 58.9 & 567.6 & 81.1 & 543.9 & vw \\
\hline $\mathrm{v}_{13}$ & $\delta(\mathrm{OCC})$ & $a^{\prime}$ & 496.9 & 2.3 & 508.2 & 2.8 & n.o. & n.o. \\
\hline $\mathrm{V}_{18}$ & $\tau\left(\mathrm{ND}_{2}\right)$ & $a^{\prime \prime}$ & 437.6 & 16.3 & 451.6 & 13.6 & n.o. & n.o. \\
\hline $\mathrm{V}_{14}$ & $\delta(\mathrm{OCN})$ & $a^{\prime}$ & 381.4 & 1.3 & 385.3 & 1.4 & n.o. & n.o. \\
\hline $\mathrm{V}_{19}$ & $\omega(\mathrm{CC})$ & $a^{\prime \prime}$ & 361.5 & 1.3 & 368.8 & 0.3 & n.o. & n.o. \\
\hline $\mathrm{V}_{20}$ & $\omega\left(\mathrm{ND}_{2}\right)$ & $a^{\prime \prime}$ & 316.5 & 78.6 & 259.0 & 98.5 & o.o.r. & o.o.r \\
\hline $\mathrm{V}_{15}$ & $\rho(\mathrm{OCN})$ & $a^{\prime}$ & 252.4 & 12.9 & 253.7 & 13.4 & o.o.r. & o.o.r. \\
\hline $\mathrm{v}_{21}$ & $\tau(\mathrm{OCN})$ & $a^{\prime \prime}$ & 68.4 & 9.8 & 59.6 & 5.2 & o.o.r. & o.o.r. \\
\hline
\end{tabular}

a: assignments ( $v=$ stretching, $\delta=$ bending, $\omega=$ wagging, $\tau=$ twisting, $\rho=$ rocking; s = symmetric, as = antisymmetric).

b: rel. experimental intensities ( $v w=$ very weak, $w=$ weak, $m=$ middle, $s=$ strong, vs = very strong; $n .0 .=$ not observed, o.o.r $=$ out of range). 
Matrix UV/Vis Spectra

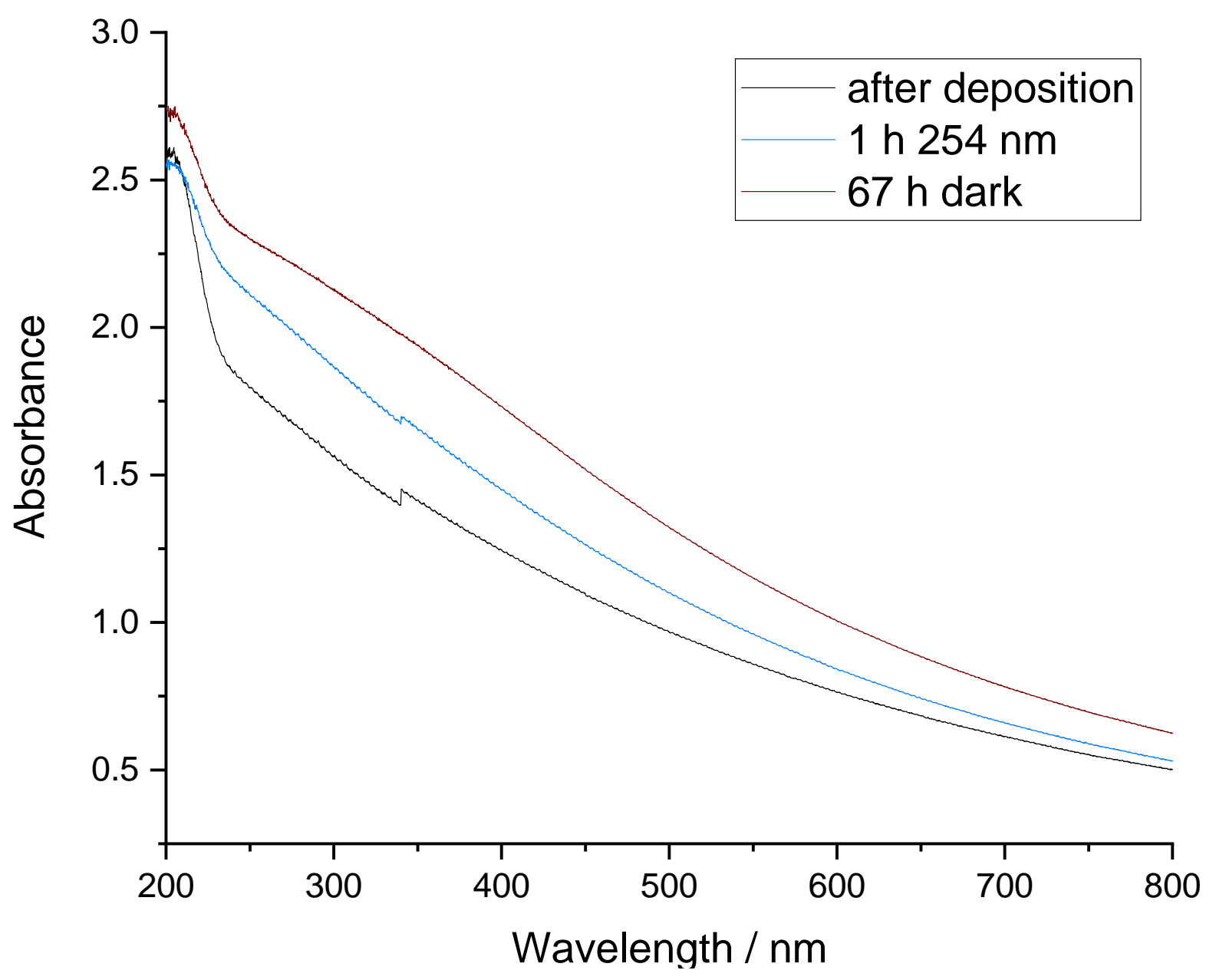

Figure S19: Matrix UV/Vis spectra recorded after deposition of oxalic acid monoamide (2) on the cold window $(3 \mathrm{~K})$ together with an excess of $\mathrm{Ar}$. 


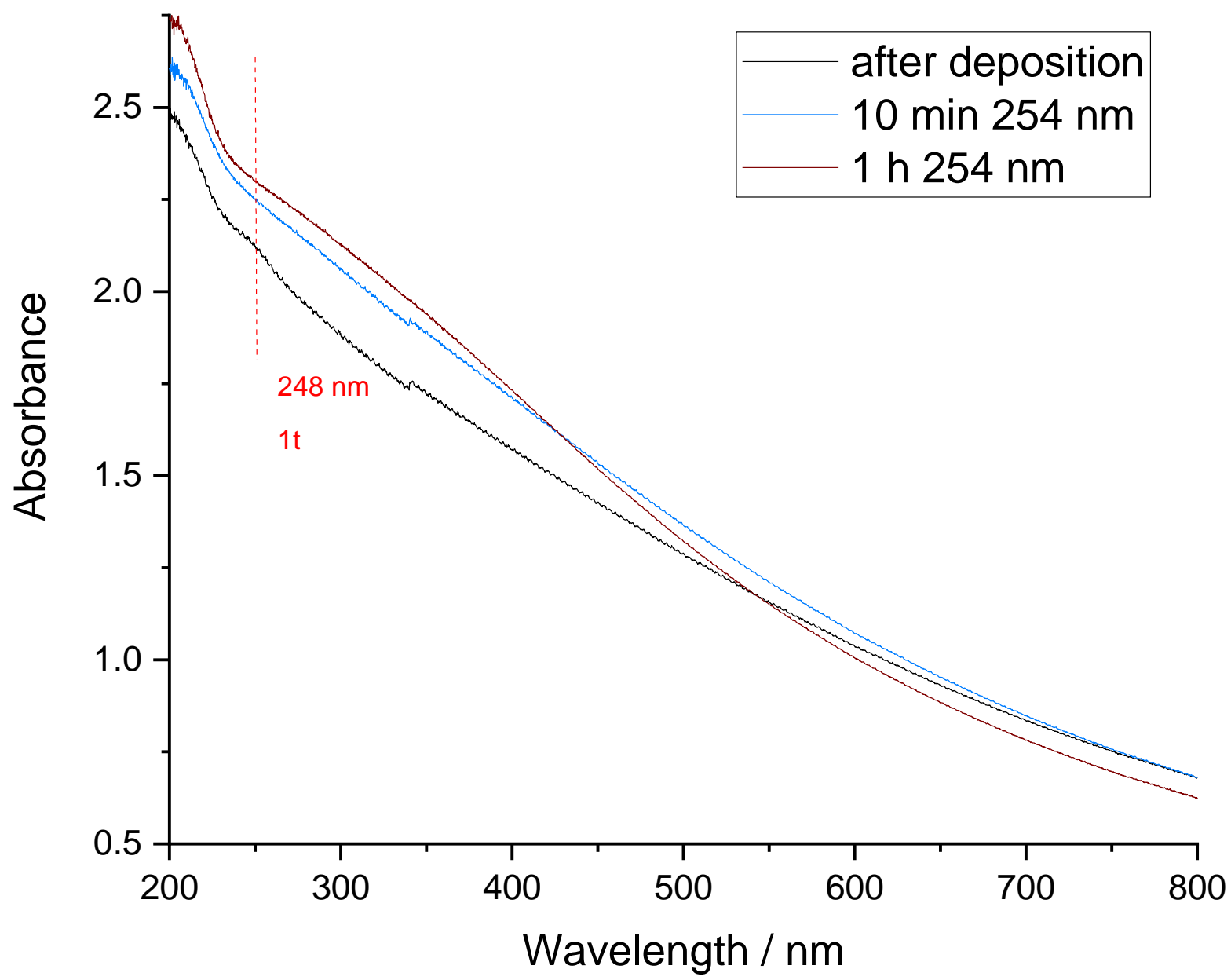

Figure S20: Matrix UV/Vis spectra after deposition of the pyrolysis products of oxalic acid monoamide (2) on the cold ( $3 \mathrm{~K}$ ) window together with an excess of Ar. A difference spectrum of the blue and the black spectra is depicted in the main text. 


\section{Computed UV/Vis Absorptions}

Table S9: TD-B3LYP/6-311++G(3df,3pd) vertical excitation energies of transaminohydroxymethylene (1t).

\begin{tabular}{lll}
\hline Excitation Energy $\lambda / \mathrm{nm}$ & Oscillator Strength $(f)$ & Transition \\
\hline 247.73 & 0.0216 & HOMO $\rightarrow$ LUMO+1 \\
242.54 & 0.0078 & HOMO $\rightarrow$ LUMO $(95.1 \%)$ \\
231.72 & & HOMO $\rightarrow$ LUMO+2 (3.5\%) \\
& 0.038 & HOMO $\rightarrow$ LUMO $+2(95.5 \%)$ \\
206.86 & & HOMO $\rightarrow$ LUMO (3.5\%) \\
179.44 & 0.1102 & HOMO $\rightarrow$ LUMO+3 \\
173.36 & 0.082 & HOMO $\rightarrow$ LUMO + 4 \\
\hline
\end{tabular}

Table S10: TD-B3LYP/6-311++G(3df,3pd) vertical excitation energies of cisaminohydroxymethylene (1c).

\begin{tabular}{lll}
\hline Excitation Energy $\lambda / \mathrm{nm}$ & Oscillator Strength $(f)$ & Transition \\
\hline 249.3 & 0.1038 & HOMO $\rightarrow$ LUMO \\
240.34 & 0.0208 & HOMO $\rightarrow$ LUMO+1 \\
223.36 & 0.0842 & HOMO $\rightarrow$ LUMO +2 \\
200.54 & 0.0571 & HOMO $\rightarrow$ LUMO+3 \\
181.55 & 0.0033 & HOMO $-1 \rightarrow$ LUMO \\
175.49 & 0.00862 & HOMO $\rightarrow$ LUMO +4 \\
\hline
\end{tabular}

Table S11: TD-B3LYP/6-311++G(3df,3pd) vertical excitation energies of cis-oxalic acid monoamide (2c).

\begin{tabular}{lll}
\hline Excitation Energy $\lambda / \mathrm{nm}$ & Oscillator Strength $(f)$ & Transition \\
\hline 296.05 & 0.0000 & HOMO $\rightarrow$ LUMO \\
207.81 & 0.0000 & HOMO $3 \rightarrow$ LUMO $(95.6 \%)$ \\
& & HOMO-1 $\rightarrow$ LUMO $(3.1 \%)$ \\
204.18 & 0.0488 & HOMO-2 $\rightarrow$ LUMO $(94.1 \%)$ \\
& & HOMO $\rightarrow$ LUMO+1 (3.0\%) \\
192.74 & 0.0112 & HOMO $\rightarrow$ LUMO+1 (93.8\%) \\
& & HOMO $\rightarrow$ LUMO+2 $(2.5 \%)$ \\
178.55 & HOMO-1 $\rightarrow$ LUMO (2.5\%) \\
& 0.0572 & HOMO-2 $\rightarrow$ LUMO (63.7\%) \\
175.02 & & HOMO $\rightarrow$ LUMO+2 $(33.2 \%)$ \\
\hline
\end{tabular}


Table S12: TD-B3LYP/6-311++G(3df,3pd) vertical excitation energies of trans-oxalic acid monoamide (2t).

\begin{tabular}{lll}
\hline Excitation Energy $\lambda / \mathrm{nm}$ & Oscillator Strength $(f)$ & Transition \\
\hline 308.13 & 0.0000 & HOMO $\rightarrow$ LUMO \\
218.66 & 0.0459 & HOMO $-1 \rightarrow$ LUMO \\
215.44 & 0.0000 & HOMO $-2 \rightarrow$ LUMO (96.1\%) \\
& & HOMO $\rightarrow$ LUMO+7 (2.6\%) \\
196.44 & 0.0095 & HOMO $\rightarrow$ LUMO+1 \\
190.46 & 0.0499 & HOMO $\rightarrow$ LUMO+2 (94.7\%) \\
& & HOMO-3 $\rightarrow$ LUMO (2.8\%) \\
181.24 & 0.0114 & HOMO-1 $\rightarrow$ LUMO+1 \\
\hline
\end{tabular}




\section{Kinetic Analyses}

As noted before, $\mathbf{2 c}$ rotamerizes to $\mathbf{2 t}$ in a QMT process. ${ }^{1}$ We recorded the kinetics of the QMT reaction $\mathbf{2 t} \rightarrow \mathbf{2} \mathbf{c}$ in four individual experiments:

A: Matrix at $3 \mathrm{~K}$, no cut-off filter between spectrometer and matrix window, 68 scans

B: Matrix at $20 \mathrm{~K}$, no cut-off filter between spectrometer and matrix window, 72 scans

C: Matrix at $3 \mathrm{~K}, 4.5 \mu \mathrm{m}$ cut-off filter between spectrometer and matrix window, 272 scans

D: Matrix at $20 \mathrm{~K}, 4.5 \mu \mathrm{m}$ cut-off filter between spectrometer and matrix window, 368 scans

Spectra were measured in intervals of $15 \mathrm{~min}$. The time dependent profiles of the most intense band of $\mathbf{2 c}\left(1737.8 \mathrm{~cm}^{-1}\right)$ and $\mathbf{2 t}\left(1751.4 \mathrm{~cm}^{-1}\right)$ were evaluated by a self-written python script, ${ }^{2-}$ 5 which we have already used in a previous project. ${ }^{6}$ The script is available at https://github.com/prs-group/Spectra_Analyzer. The script calculates the area $y$ between the band and a straight line between two selected points on either side of the band. The area $y$ is fitted against time $t$ by an exponential function $y=A \cdot \exp (-k \cdot t)+B$. The half-life is then given by $t_{1 / 2}=\ln (2) / k$.

Table S13: Experimental tunneling half-lives of the reaction $\mathbf{2 t} \rightarrow \mathbf{2 c}$ obtained by evaluation of the strongest bands at $1751.4(2 \mathrm{t})$ and $1737.8 \mathrm{~cm}^{-1}(\mathbf{2 c})$, respectively.

\begin{tabular}{cccc}
\hline Experimental Conditions & $t_{1 / 2}(\mathbf{2 t}) / \mathrm{h}$ & $t_{1 / 2}(\mathbf{2 c}) / \mathrm{h}$ & Mean $/ \mathrm{h}$ \\
\hline A & 2.1 & 3.3 & $2.70 \pm 0.60$ \\
B & 1.7 & 2.0 & $1.85 \pm 0.15$ \\
C & 15.8 & 15.5 & $15.65 \pm 0.15$ \\
D & 6.7 & 5.9 & $6.30 \pm 0.40$ \\
\hline
\end{tabular}




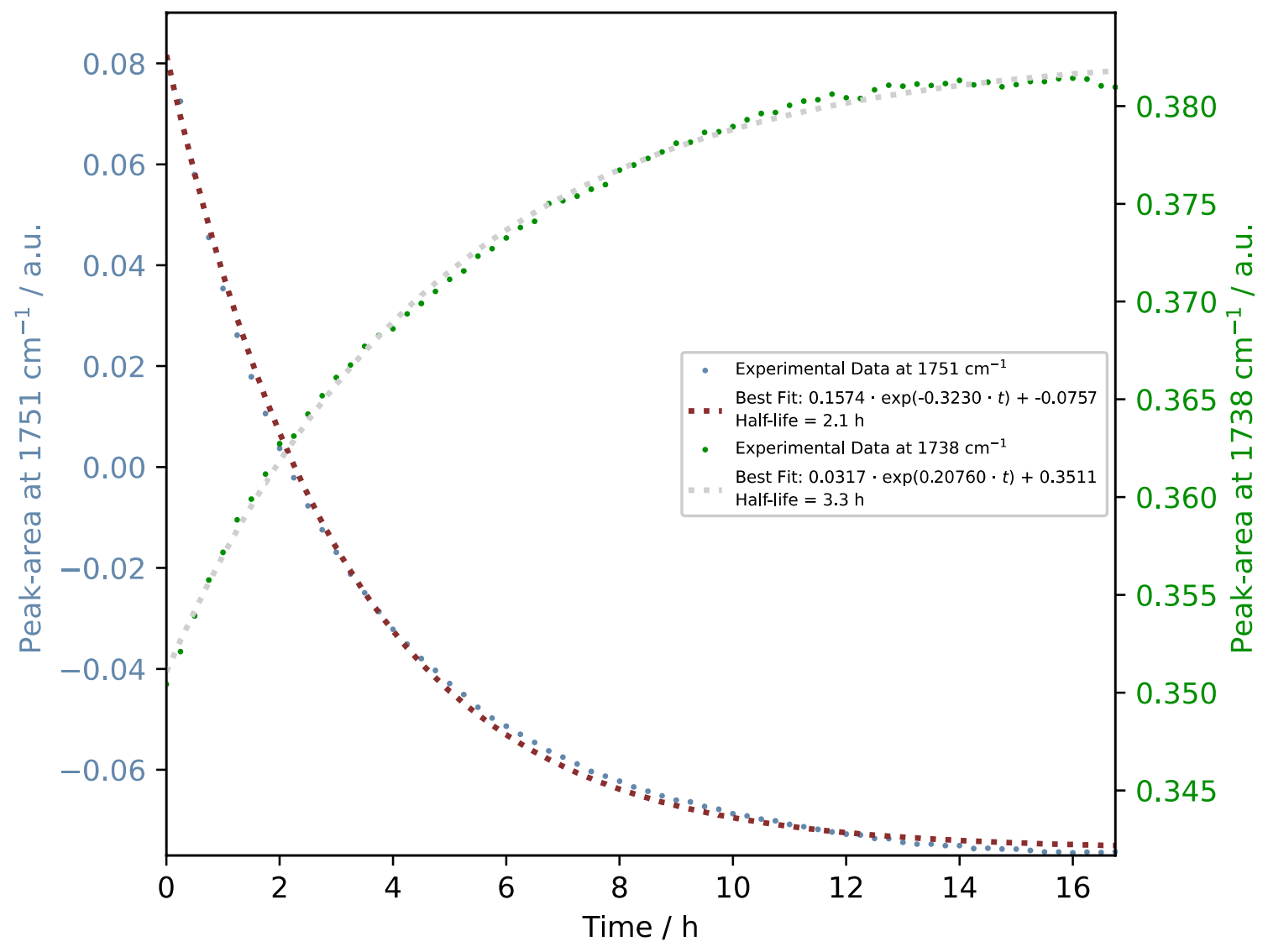

Figure S21: Experiment A: Evaluation of the bands at $1751 \mathrm{~cm}^{-1}(\mathbf{2 t})$ and $1738 \mathrm{~cm}^{-1}(\mathbf{2 c})$. 


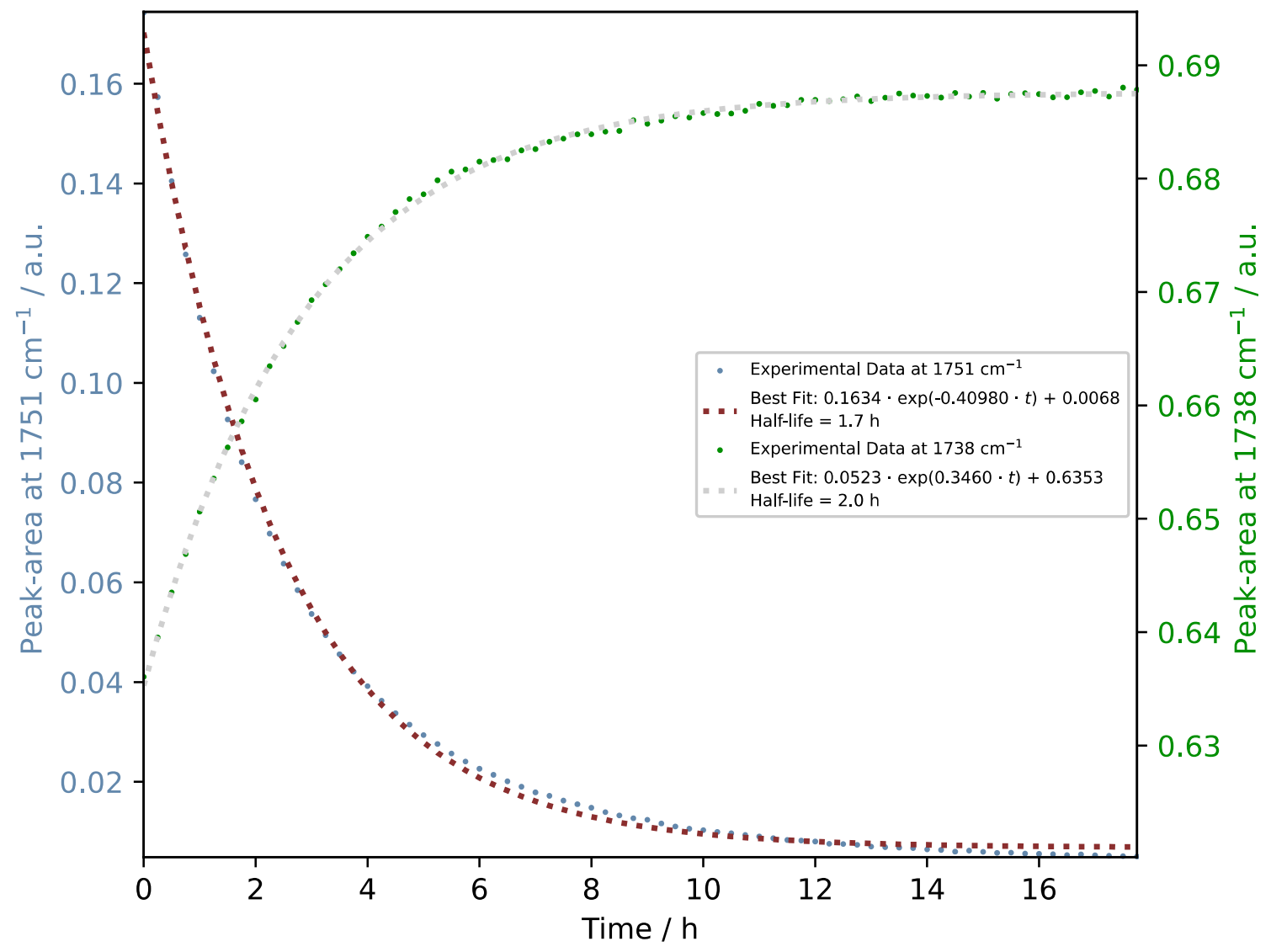

Figure S22: Experiment B: Evaluation of the bands at $1751 \mathrm{~cm}^{-1}(\mathbf{2 t})$ and $1738 \mathrm{~cm}^{-1}(\mathbf{2 c})$. 


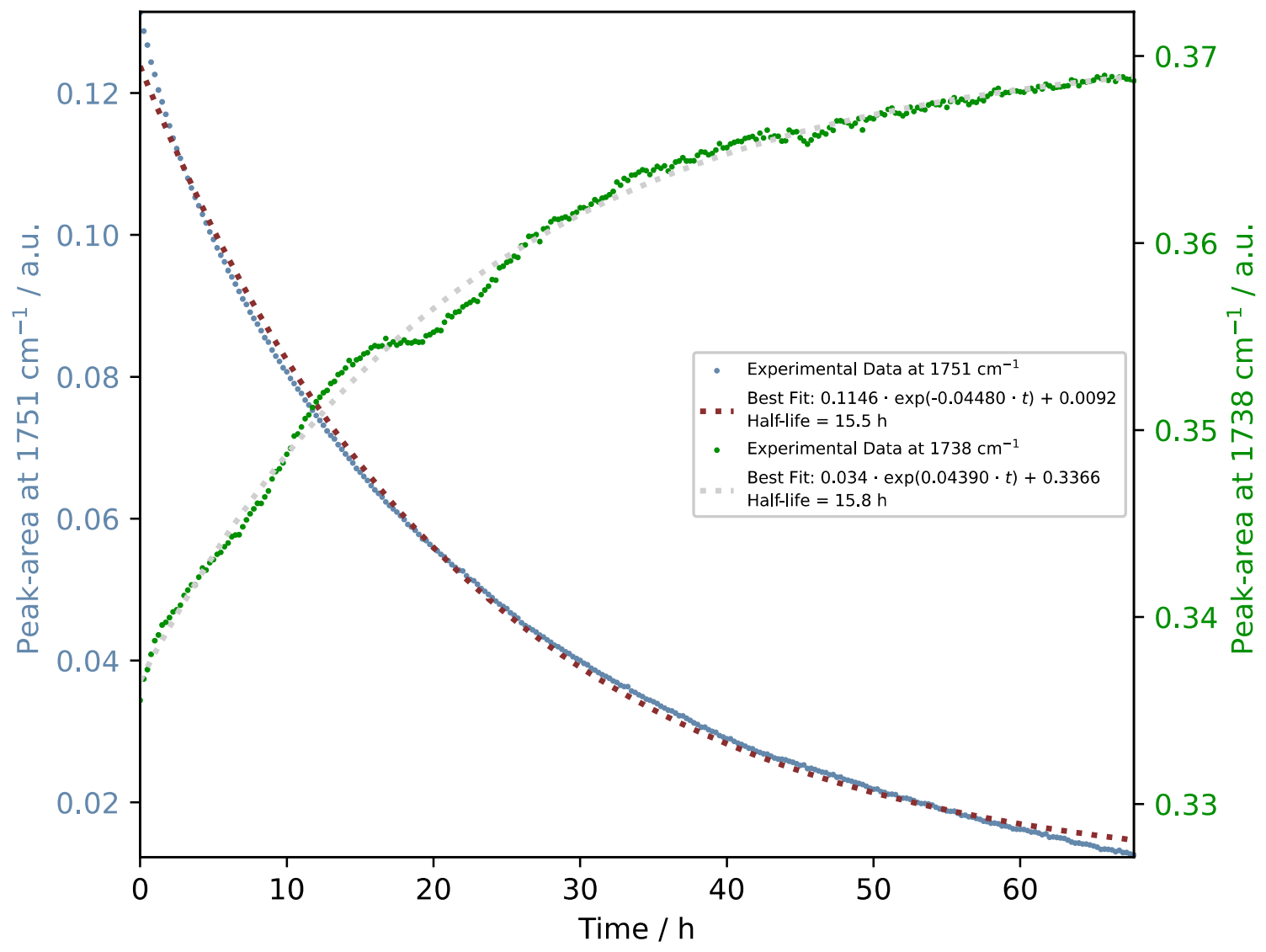

Figure S23: Experiment C: Evaluation of the bands at $1751 \mathrm{~cm}^{-1}(\mathbf{2 t})$ and $1738 \mathrm{~cm}^{-1}(\mathbf{2 c})$. 


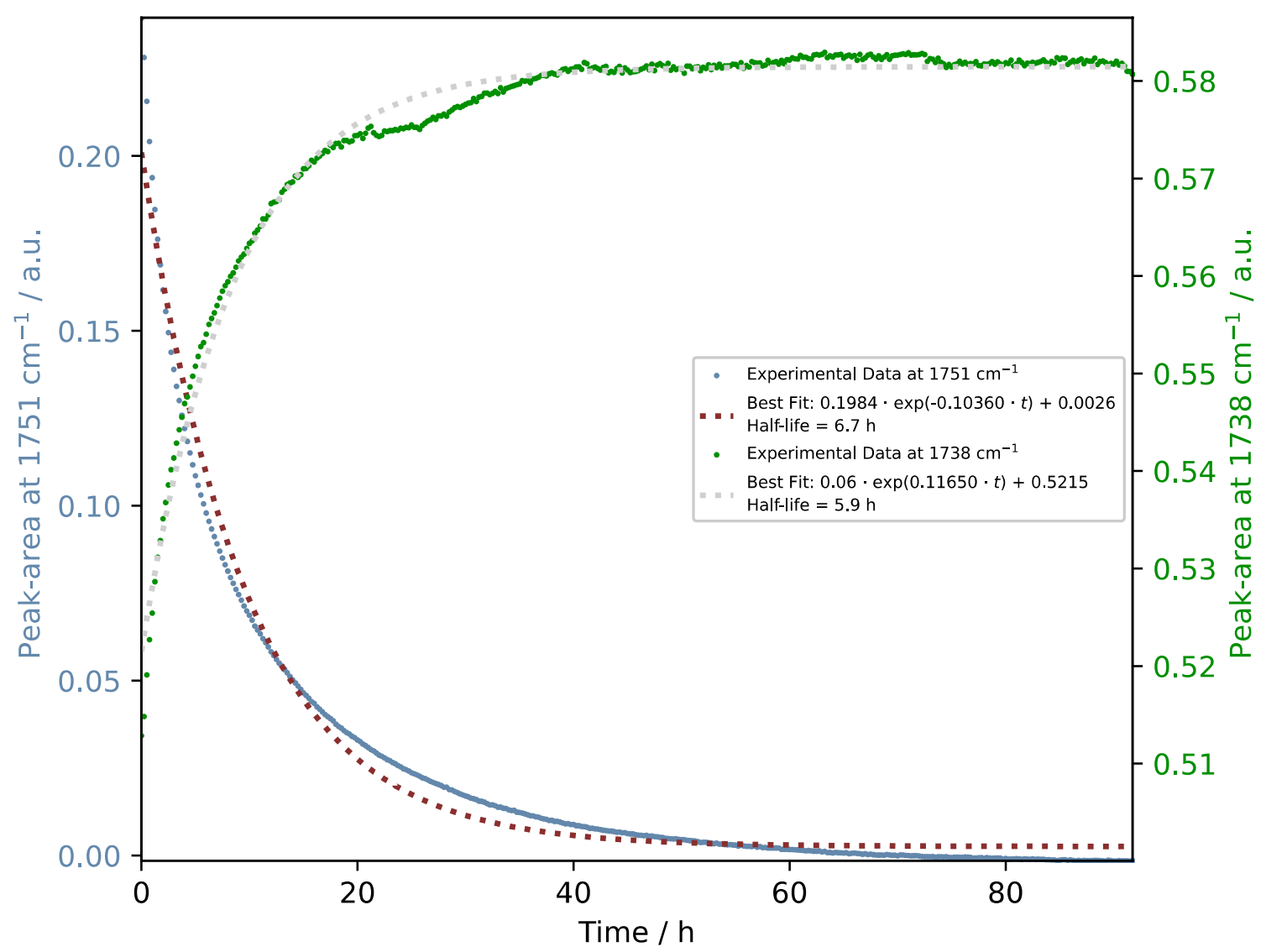

Figure S24: Experiment D: Evaluation of the bands at $1751 \mathrm{~cm}^{-1}(\mathbf{2 t})$ and $1738 \mathrm{~cm}^{-1}(\mathbf{2 c})$.

While the QMT half-lives measured without using a cut-off filter (experiments $\mathbf{A}$ and $\mathbf{B}$ ) are essentially the same (ca. 3 and $2 \mathrm{~h}$, respectively), the half-lives in C (ca. $16 \mathrm{~h}$ ) and D (ca. $6 \mathrm{~h}$ ) are noticeably different. The spectrometer beam apparently has an impact on the reaction $\mathbf{2 t} \rightarrow \mathbf{2 c}$. The intrinsic QMT behavior of the reaction is presumably best captured in experiment C, where both thermal and photon excitation are less abundant. However, the rather minute temperature dependence is a strong hint for QMT being the crucial factor for the $\mathbf{2 t} \rightarrow \mathbf{2 c}$ reaction. 


\section{Computations}

All DFT computations were performed with the Gaussian 16, Revision C.01 program package (full citations for electronic structure codes are given at the end of this document) at the B3LYP $7-8 / 6-311++G(3 d f, 3 p d)^{9-10}$ level of theory. The keywords Opt and Freq=NoRaman were used for the characterization of minima on the PES. For transition structures the keyword Opt $=($ TS,CalcAll,NoEigen,VTight,MaxCyc $=50)$ was used. IRC computations were conducted with IRC $=($ CalcAll,Forward, MaxPoints $=1000$, MaxCycle $=100$,StepSize $=5$, phase $=(W, X, Y, Z)$ ). UV/Vis absorptions were computed by using the keyword $T D=($ Root $=1$, NStates $=6)$. Anharmonic frequencies were computed by using Freq=(Anharmonic,NoRaman).

Coupled-cluster computations performed with CFOUR were carried out at the CCSD(T) $11-15 / \mathrm{cc}$ pVTZ ${ }^{16-20}$ level of theory and the following keywords: FROZEN_CORE $=1$, CONVERGENCE $=8$, $A B C D T Y P E=A O B A S I S, C C \_P R O G=E C C, S C F \_C O N V=8$, and $C C \_C O N V=10$. Harmonic frequencies were computed analytically with $\mathrm{VIB}=\mathrm{EXACT}$; for transition structures the keyword METHOD=TS was used additionally.

Computational tunneling half-lives were determined at the CVT/SCT//B3LYP/6$311++G(3 d f, 3 p d)$ level of theory using Polyrate Version 2017-C.

Natural bond orbitals (NBO), ${ }^{21}$ natural resonance theory (NRT), and Wiberg bond indices were computed with NBO7 at the B3LYP/6-311++G(3df,3pd) level of theory based on geometries at the same level. 


\section{Computed Potential Energy Surfaces}<smiles>NC(=O)C(=O)O</smiles>

2c

0.0

0.0<smiles>NC(=O)C(=O)O</smiles>

$2 \mathrm{t}$

3.8

3.8<smiles>NC(=O)C(=O)O</smiles>

$2 \mathbf{t}^{\prime}$

6.0

6.0<smiles>NC(=O)C(=O)O</smiles>

2c'

$(-)$

Scheme S1: There are three Cs conformers of oxalic acid monoamide (2). Among them 2c is the most stable one. Conceivable conformer $\mathbf{2} \mathbf{c}^{\prime}$ could not be located as a local minimum structure. Relative energies including ZPVEs are given in $\mathrm{kcal} \mathrm{mol}^{-1}$ at the B3LYP/6$311++G(3 d f, 3 p d)$ (black) and the CCSD(T)/cc-pVTZ (blue) levels of theory.

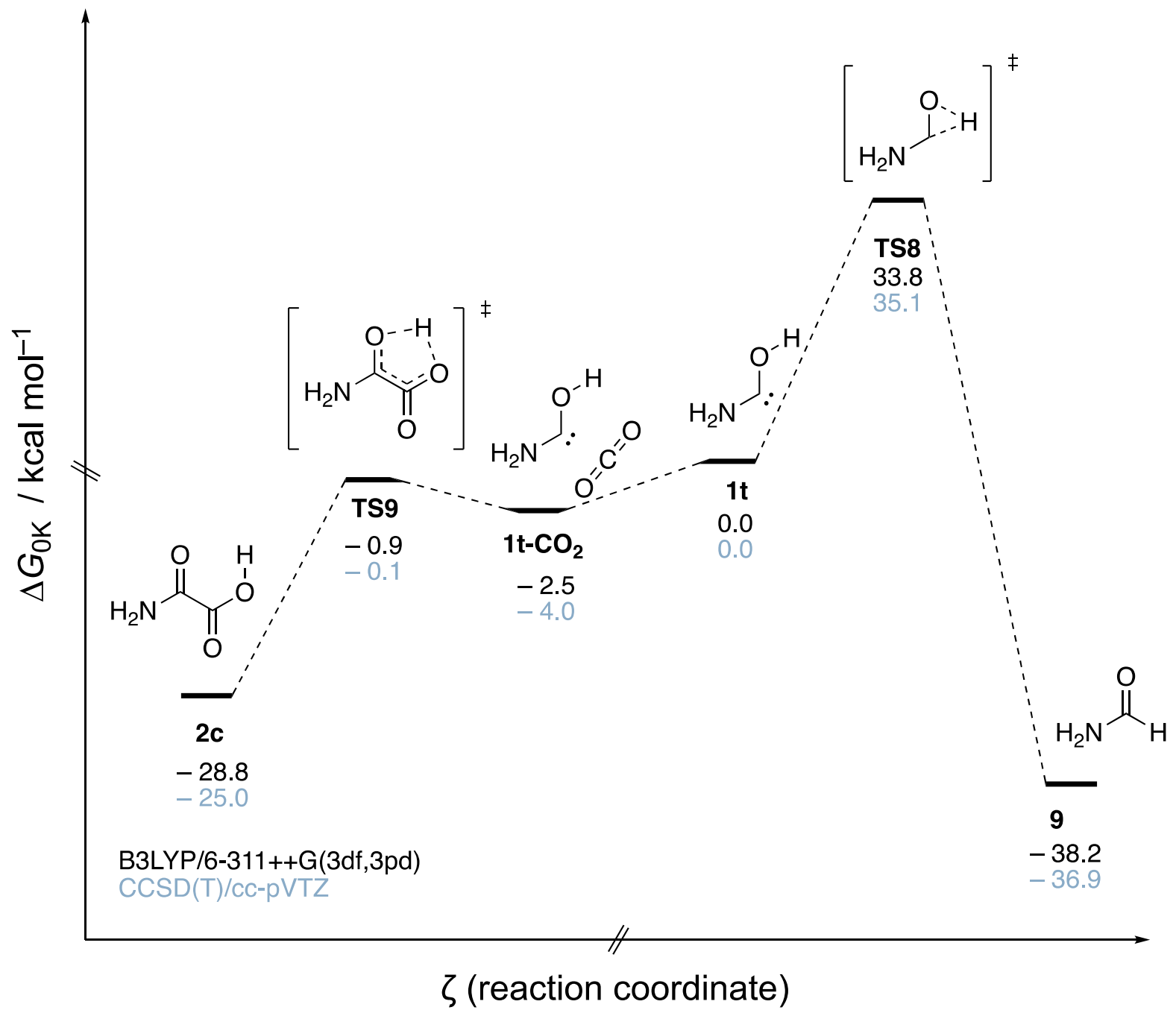

Scheme S2: PES of the pyrolysis of $\mathbf{2 c}$ via $\mathbf{1 t}-\mathrm{CO}_{\mathbf{2}}$ and $\mathbf{1 t}$ to yield formamide (9) at $0 \mathrm{~K}$. All energies include ZPVEs computed at the corresponding level of theory. 


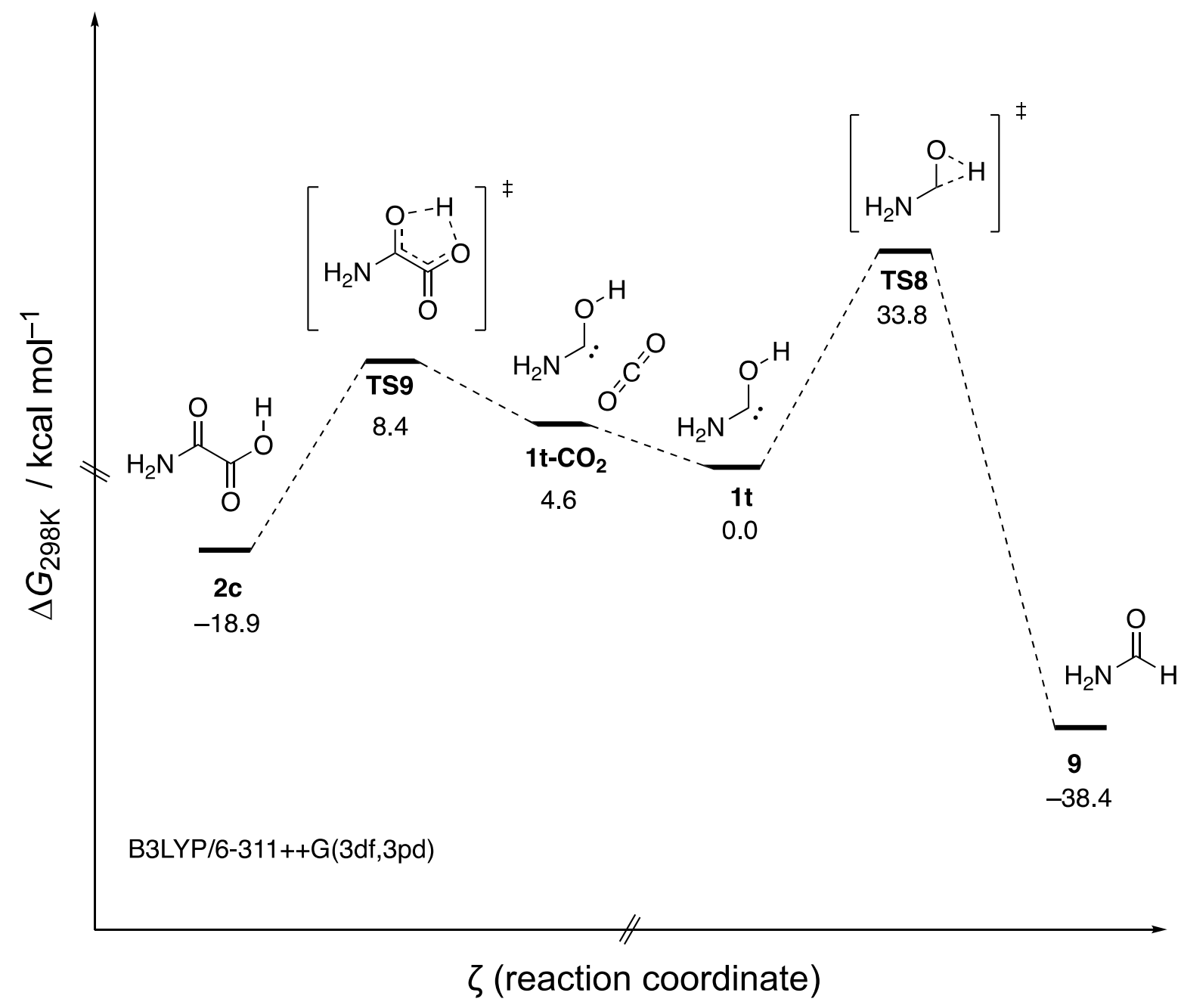

Scheme S3: PES of the pyrolysis of $\mathbf{2 c}$ via $\mathbf{1 t}-\mathrm{CO}_{2}$ and $\mathbf{1 t}$ to yield formamide (9) at $298.15 \mathrm{~K}$. All energies include ZPVEs computed at the corresponding level of theory. 


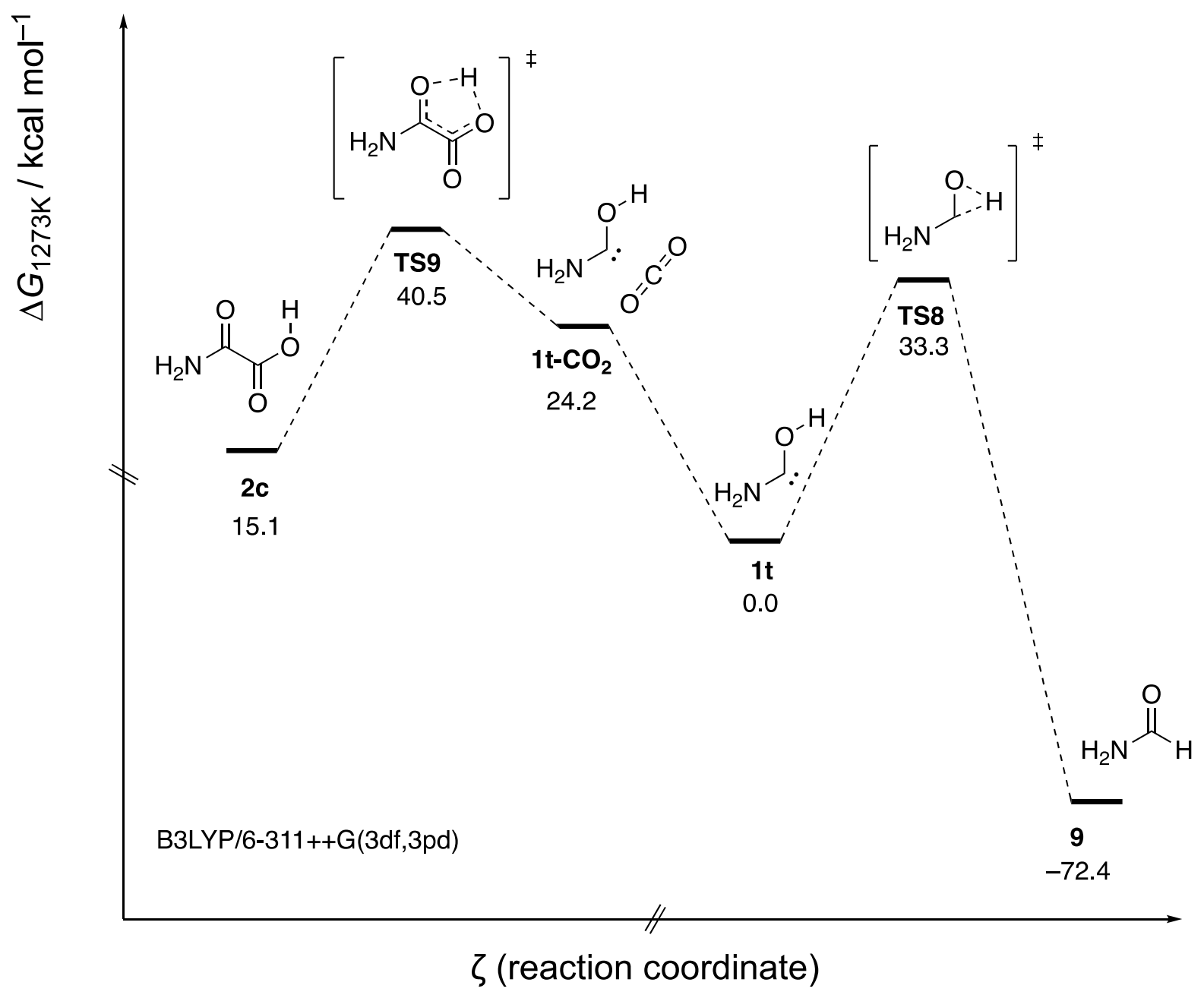

Scheme S4: PES of the pyrolysis of $\mathbf{2 c}$ via $\mathbf{1 t}-\mathrm{CO}_{2}$ and $\mathbf{1 t}$ to yield formamide (9) at $1273.15 \mathrm{~K}$. All energies include ZPVEs computed at the corresponding level of theory. 


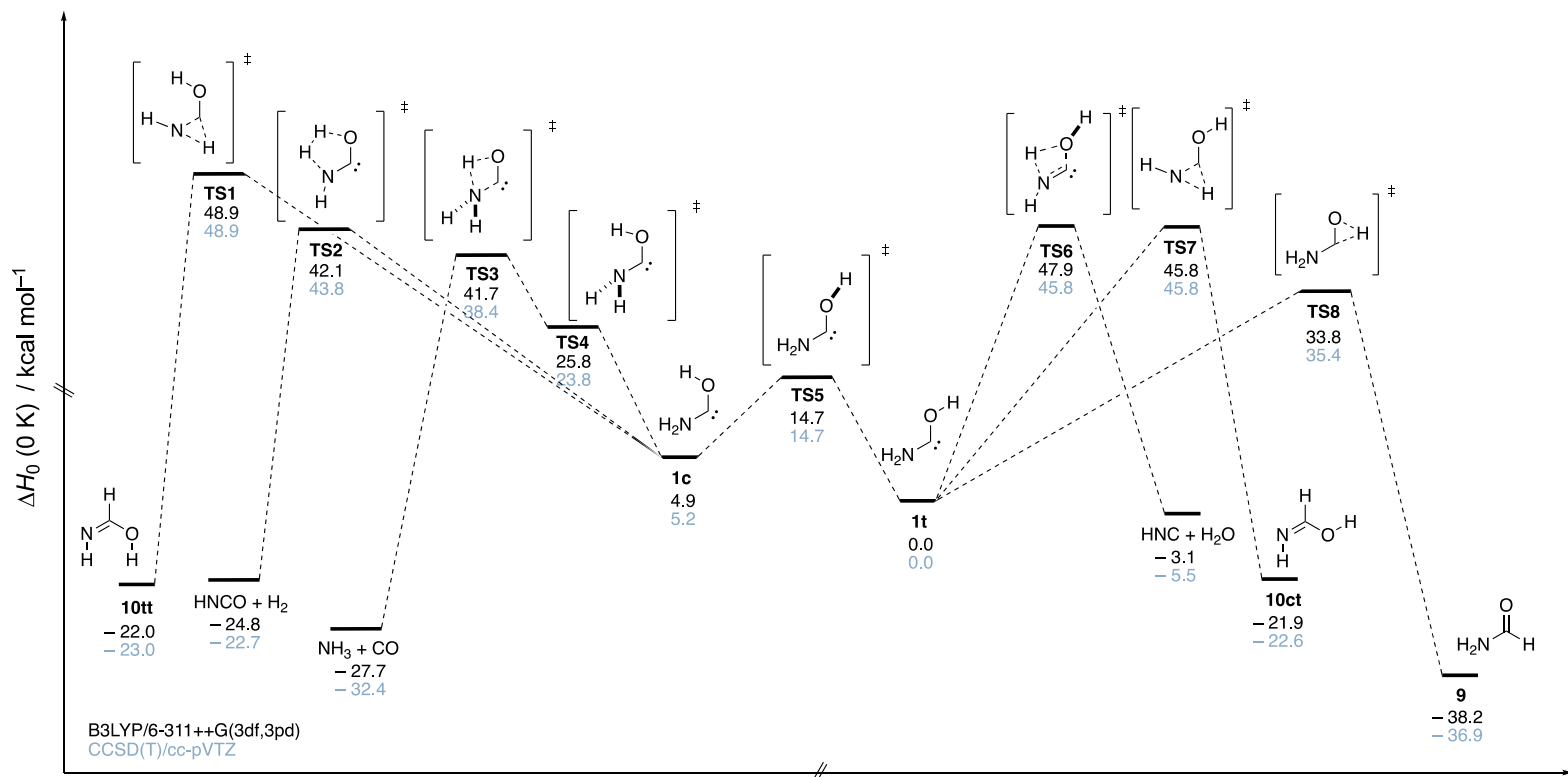

$\zeta$ (reaction coordinate)

Scheme S5: PES around aminohydroxymethylene (1). All energies include ZPVEs computed at the corresponding level of theory.

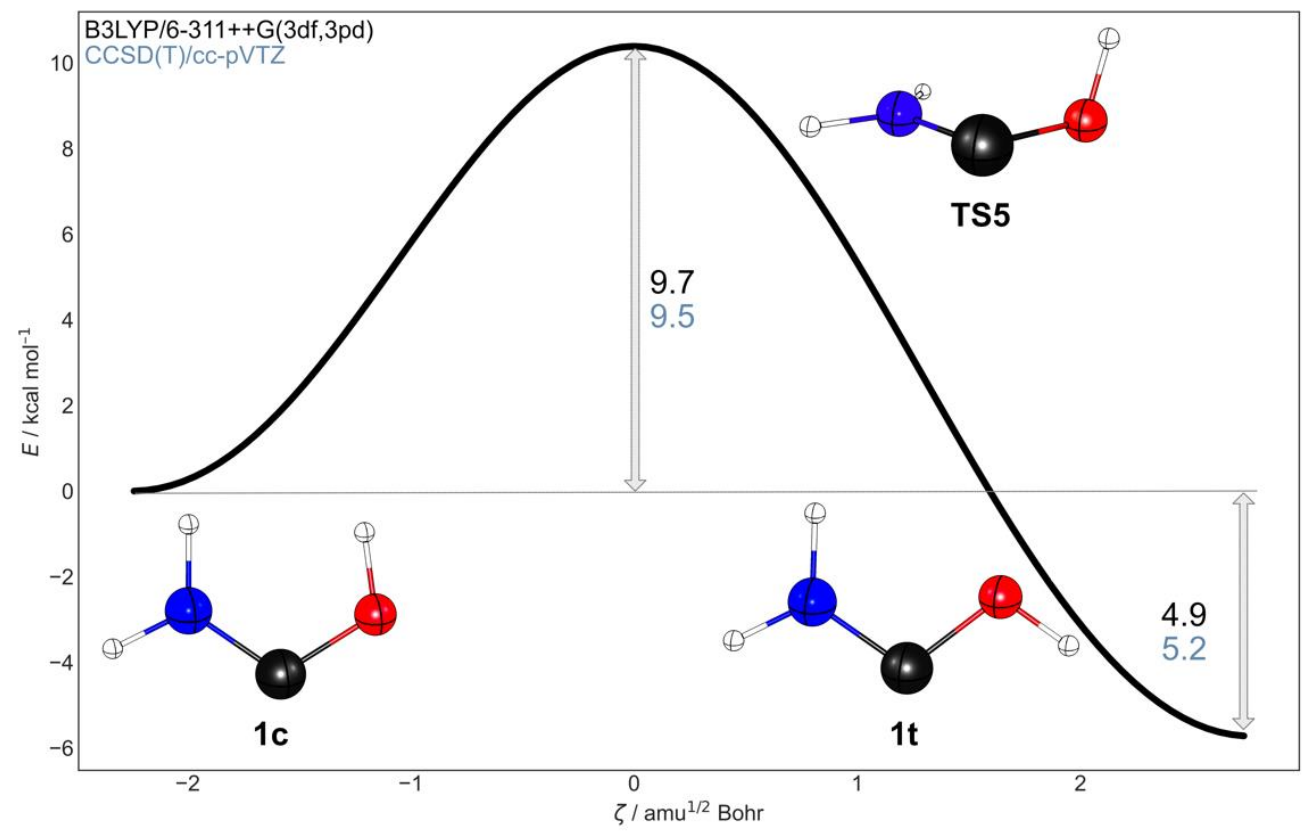

Scheme S6: IRC of the rotamerization of 1 computed at the B3LYP/6-311++G(3df,3pd) level of theory. This reaction is associated with a computed QMT half-life of $2.7 \mathrm{~s}$ (vide infra) and, hence, 1c cannot be observed in our experiments. 


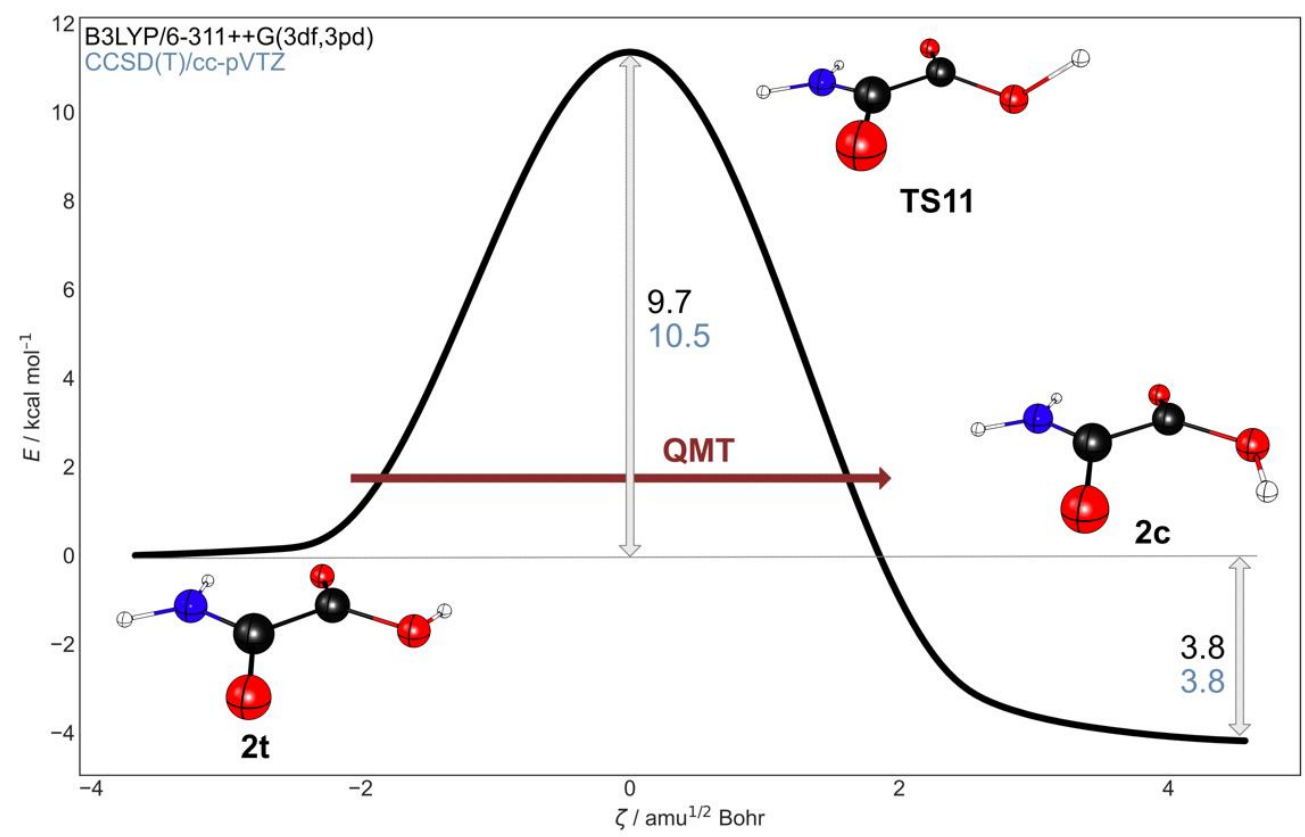

Scheme S7: IRC of the rotamerization of 2 computed at the B3LYP/6-311++G(3df,3pd) level of theory. This reaction is associated with a QMT half-life of ca. $2 \mathrm{~h}$ at $3 \mathrm{~K}$ (comput. $15.6 \mathrm{~min}$; vide infra).

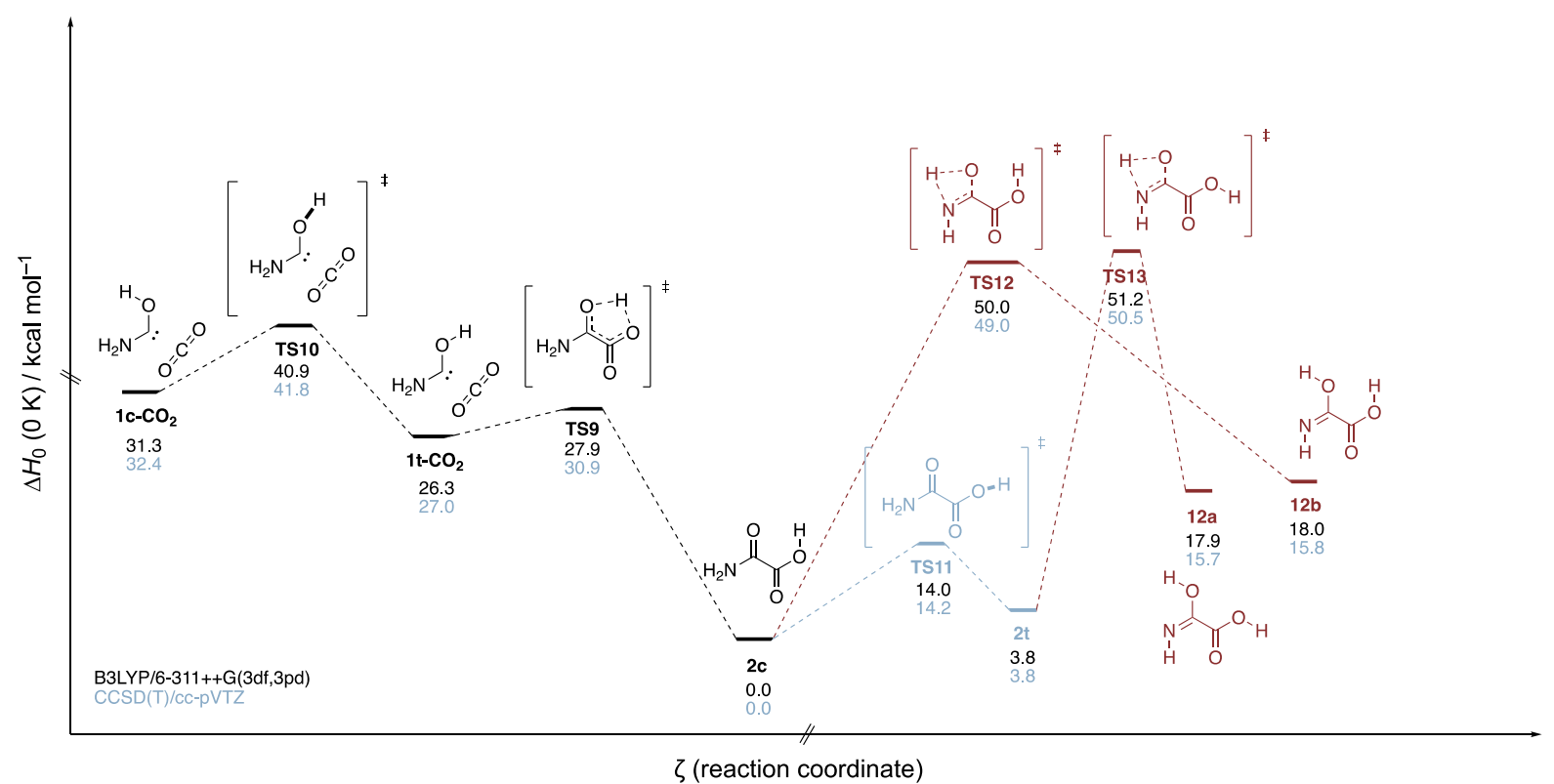

Scheme S8: Conceivable reaction channels of 2. Experimentally, only the rotamerization $\mathbf{2 c} \rightarrow \mathbf{2 t}$ and the reverse process due to QMT were observed. All energies include ZPVEs computed at the corresponding level of theory. 


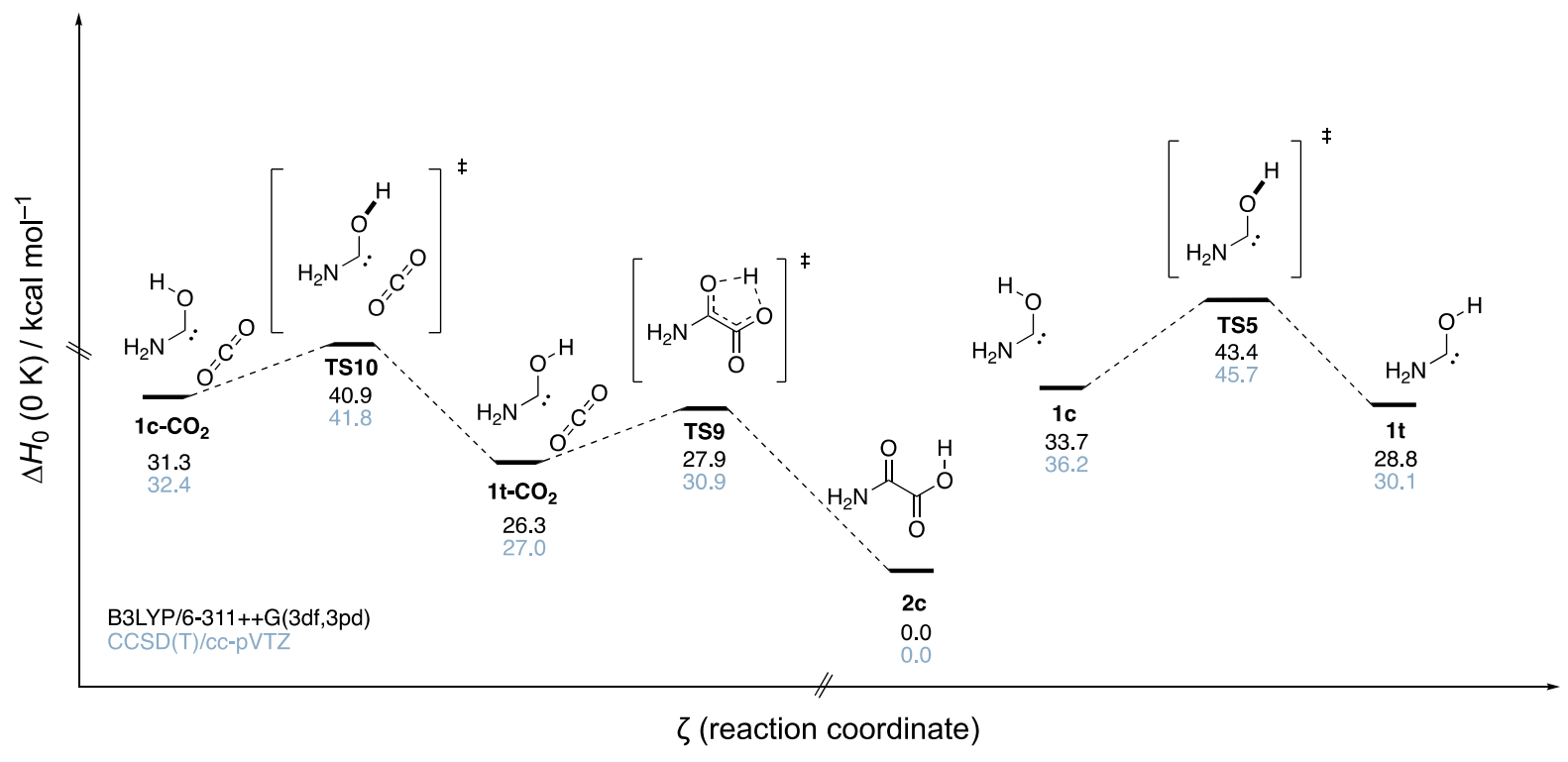

Scheme S9: Comparison of the PESs of $\mathbf{1 t}-\mathrm{CO}_{2}$ (left) and free $\mathbf{1}$ (right). The energetics of the right PES were obtained by adding the energy of $\mathrm{CO}_{2}$ computed at the respective level of theory. The carbene $\mathrm{CO}_{2}$ complex is stabilized by ca. $3 \mathrm{kcal} \mathrm{mol}^{-1}$ compared to free 1 . All energies include ZPVEs computed at the corresponding level of theory.

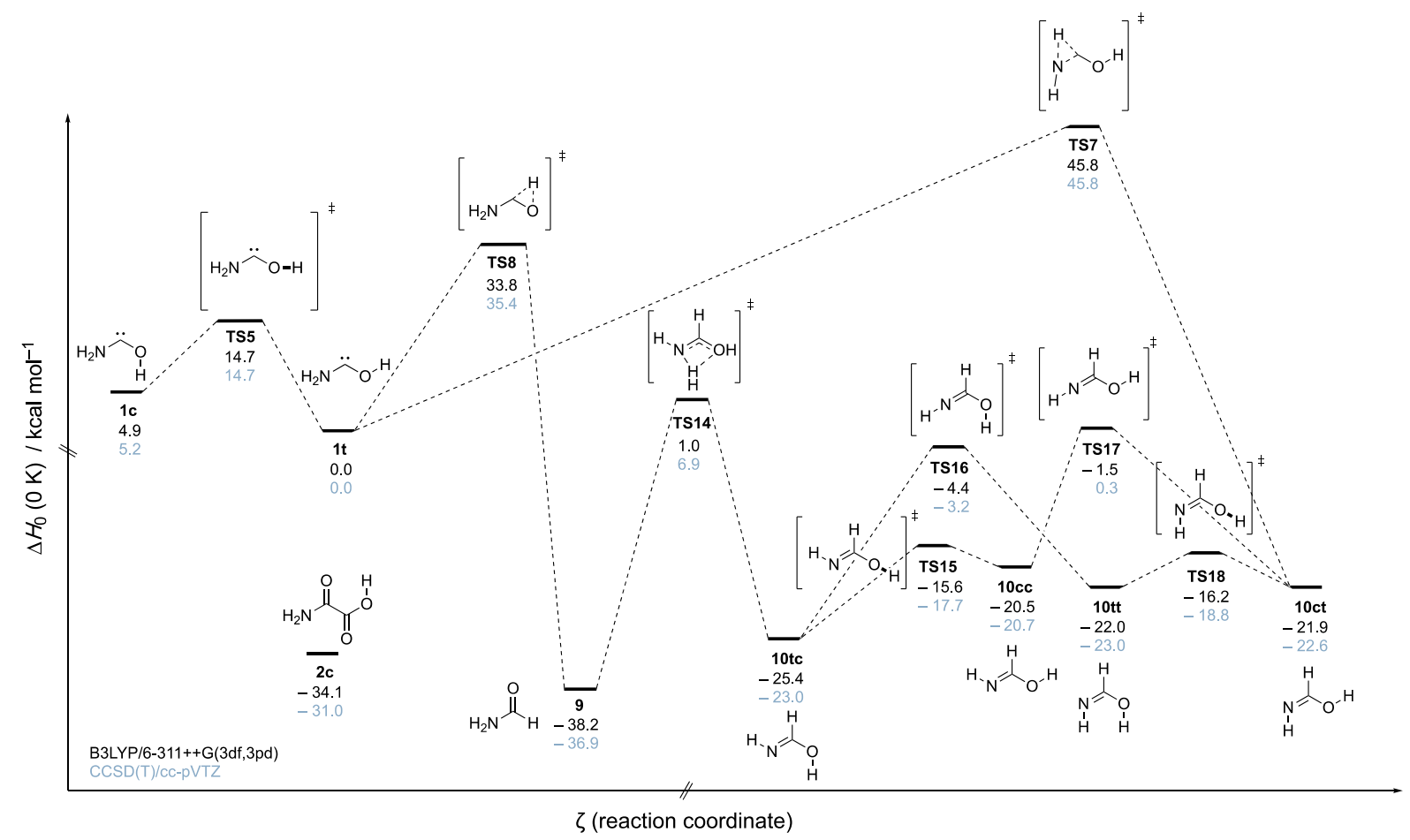

Scheme S10: PES connecting 1 and 10. All energies include ZPVEs computed at the corresponding level of theory. 


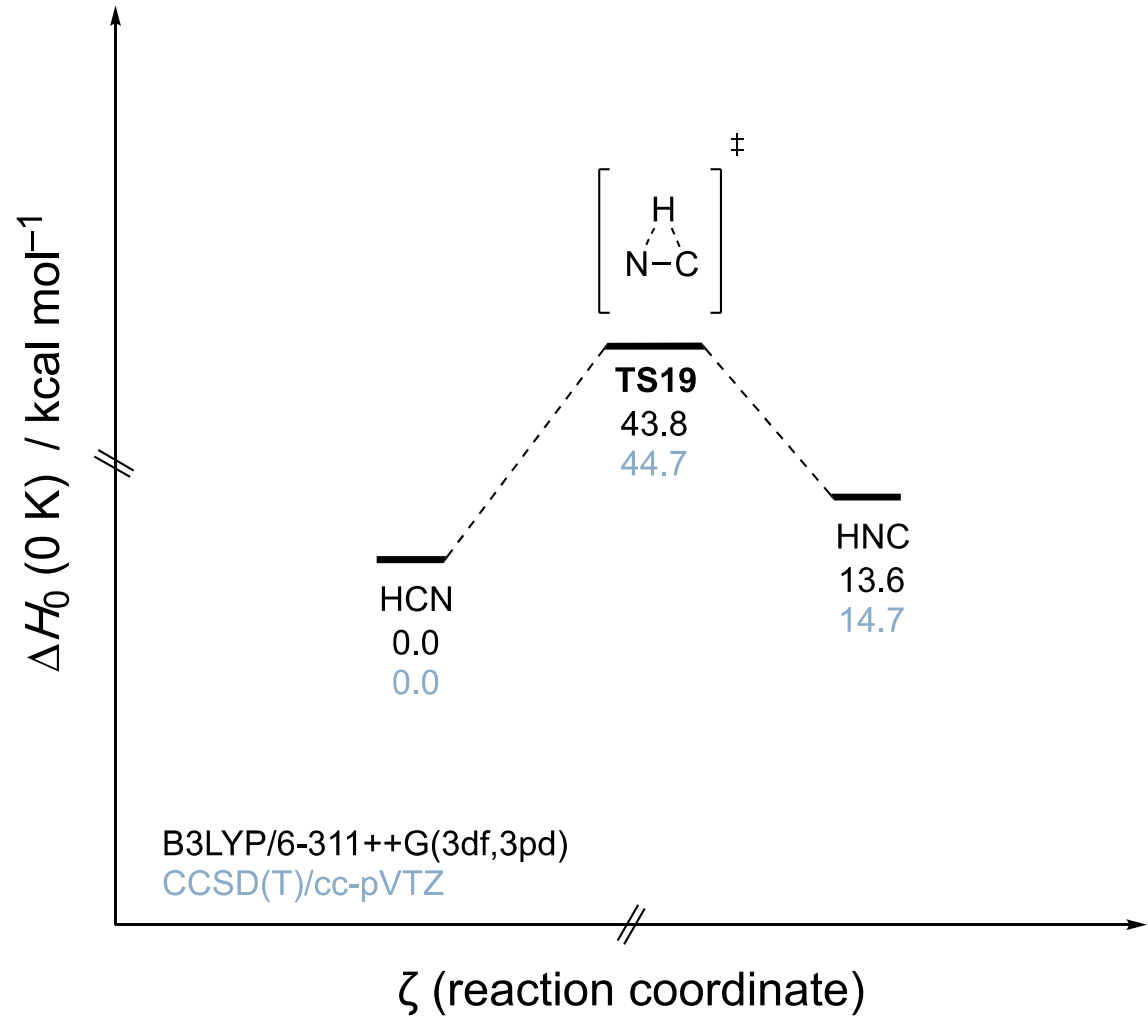

Scheme S11: PES of the isomerization between HCN and HNC. All energies include ZPVES computed at the corresponding level of theory. 
<smiles>NC(=O)C(=O)O</smiles>

2c<smiles>[2H]O/C(=N/N)C(=O)O</smiles>

$12 f$<smiles>O=C(O)/C(O)=N\O</smiles>

$12 a$<smiles>N=C(O)C(=O)O</smiles>

$12 b$<smiles>O=C(O)/C(O)=N\O</smiles>

$12 \mathrm{c}$<smiles></smiles>

12d<smiles>N=C(O)C(=O)O</smiles>

$12 e$

$$
\begin{array}{lll}
23.5 & 28.1 & 18.9 \\
21.8 & (-) & 16.6
\end{array}
$$<smiles>N=C(O)C(=O)O</smiles>

12j

$\begin{array}{lllll}18.5 & 20.1 & 24.4 & 24.4 & 13.5 \\ 16.4 & 18.0 & 22.6 & 22.3 & 11.4\end{array}$

12k

13.5
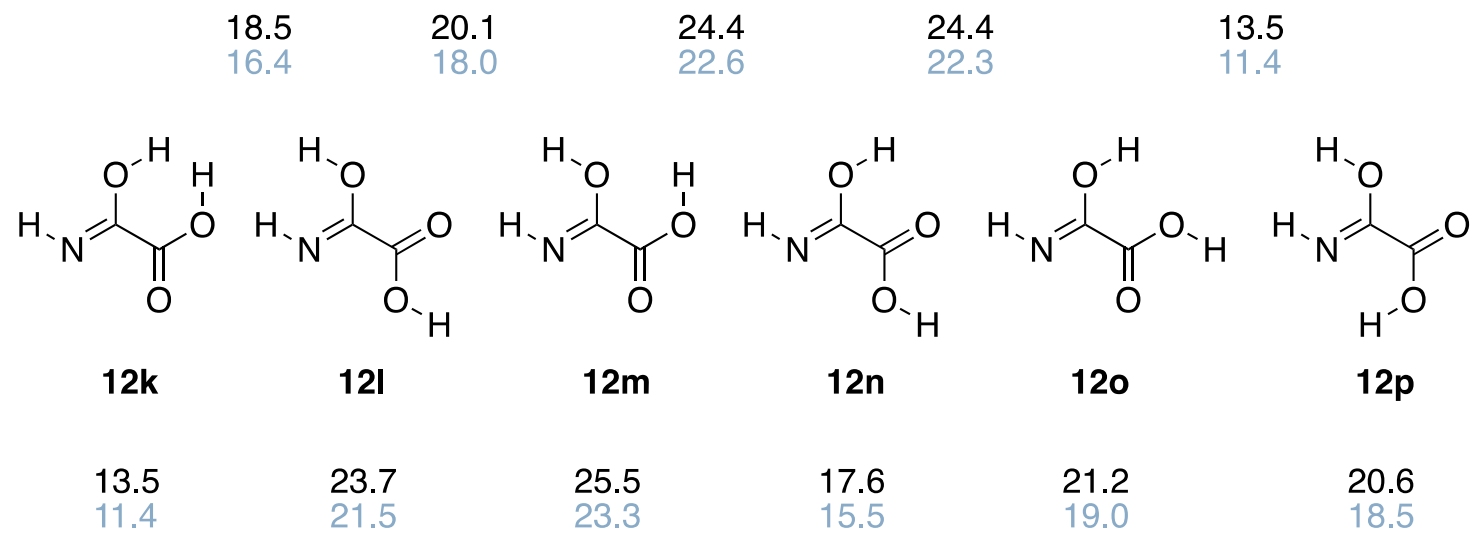<smiles>N=C(O)C(=O)O</smiles>

$12 g$<smiles></smiles>

$12 \mathrm{~h}$<smiles>N=C(O)C(=O)O</smiles>

$12 i$

Scheme S12: There are 16 conformers of the enolimine tautomer (12) of 2. Relative energies including ZPVEs are given in $\mathrm{kcal} \mathrm{mol}^{-1}$ at the B3LYP/6-311++G(3df,3pd) (black) and the CCSD(T)/cc-pVTZ (blue) levels of theory at $0 \mathrm{~K}$. However, we did not observe any such tautomer in our experiments. 
$\mathrm{BDE} / \mathrm{kcal} \mathrm{\textrm {mol } ^ { - 1 }}$

B3LYP/6-311++G(3df,3pd)

$\mathrm{H}$<smiles>[OH+]=CN[Hg]</smiles>

$\longrightarrow \mathrm{H}_{2} \mathrm{NCO}+\mathrm{H}$

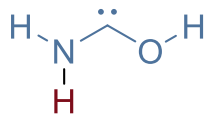

$\longrightarrow$

trans- $\mathrm{HNCOH}+\mathrm{H} \cdot$

$\mathrm{H}_{\substack{\mathrm{N} \\ \mathrm{H}}} \overbrace{}^{-} \mathrm{O}^{-}$

$\longrightarrow \quad \mathrm{Cis}-\mathrm{HNCOH}+\mathrm{H} \bullet$

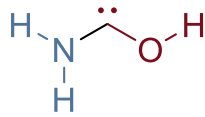

$\longrightarrow \quad \mathrm{H}_{2} \mathrm{~N} \cdot+\cdot \mathrm{COH}$

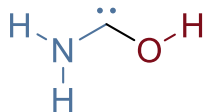

$\mathrm{H}_{2} \mathrm{NC} \cdot+\cdot \cdot \mathrm{OH}$
51.7

72.3

77.4

97.5

98.4

Scheme S13: Conceivable mechanisms from 1 to $\mathrm{NH}_{3}+\mathrm{CO}$ and $\mathrm{HNCO}+\mathrm{H}_{2}$ involving radical intermediates would necessarily involve homolytic bond cleavage in 1 . However, all bond dissociation energies (BDEs) are higher in energy than the transition states in Scheme S5. Nevertheless, they are reachable upon irradiation at $254 \mathrm{~nm}$. All energies include ZPVEs computed at the corresponding level of theory. 


\section{Tunneling Computations}

Table S14: Overview of computed tunneling half-lives at the CVT/SCT//B3LYP/6$311++G(3 d f, 3 p d)$ level of theory.

\begin{tabular}{llll}
\hline Reaction & Symmetry Number & Tunneling Half-Life & Kinetic Isotope Effect \\
\hline $\mathbf{1 c} \rightarrow \mathbf{1 t}$ & 2 & $2.7 \mathrm{~s}$ & $8.3 \cdot 10^{5}$ \\
$\boldsymbol{d}_{\mathbf{1}} \mathbf{- 1} \mathbf{c} \rightarrow \boldsymbol{d}_{\mathbf{1}} \mathbf{- 1 t}$ & 2 & $615.0 \mathrm{~h}$ & \\
$\mathbf{2 t} \rightarrow \mathbf{2 c}$ & 2 & $15.6 \mathrm{~min}$ & $2.4 \cdot 10^{6}$ \\
$\boldsymbol{d}_{\mathbf{1}} \mathbf{- 2} \mathbf{c} \rightarrow \boldsymbol{d}_{\mathbf{1}} \mathbf{- 2} \mathbf{t}$ & 2 & $70.2 \mathrm{a}$ & \\
$\mathbf{1 t} \rightarrow \mathbf{9}$ & 1 & $167.8 \mathrm{~d}$ & $7.9 \cdot 10^{7}$ \\
$\boldsymbol{d}_{\mathbf{1}} \mathbf{- 1 t} \rightarrow \boldsymbol{d}_{\mathbf{1}} \mathbf{- 9}$ & 1 & $3.6 \cdot 10^{7} \mathrm{a}$ & \\
\hline
\end{tabular}

Table S15: Rate constants of the reaction $1 \mathrm{c} \rightarrow \mathbf{1 t}$ in $\mathrm{s}^{-1}$ computed at the B3LYP/6$311++G(3 d f, 3 p d)$ level of theory.

\begin{tabular}{lllll}
\hline$T / \mathrm{K}$ & $\mathrm{TST}$ & $\mathrm{CVT}$ & $\mathrm{CVT} / \mathrm{ZCT}$ & $\mathrm{CVT} / \mathrm{SCT}$ \\
\hline 0.10 & $\mathrm{NaN}$ & $\mathrm{NaN}$ & $\mathrm{NaN}$ & $\mathrm{NaN}$ \\
8.00 & $3.75 \cdot 10^{-255}$ & $3.75 \cdot 10^{-255}$ & $1.32 \cdot 10^{-3}$ & $2.59 \cdot 10^{-1}$ \\
10.00 & $7.30 \cdot 10^{-202}$ & $7.30 \cdot 10^{-202}$ & $1.32 \cdot 10^{-3}$ & $2.59 \cdot 10^{-1}$ \\
15.00 & $9.18 \cdot 10^{-131}$ & $9.19 \cdot 10^{-131}$ & $1.32 \cdot 10^{-3}$ & $2.59 \cdot 10^{-1}$ \\
20.00 & $3.55 \cdot 10^{-95}$ & $3.55 \cdot 10^{-95}$ & $1.32 \cdot 10^{-3}$ & $2.59 \cdot 10^{-1}$ \\
25.00 & $8.39 \cdot 10^{-74}$ & $8.39 \cdot 10^{-74}$ & $1.32 \cdot 10^{-3}$ & $2.59 \cdot 10^{-1}$ \\
30.00 & $1.54 \cdot 10^{-59}$ & $1.54 \cdot 10^{-59}$ & $1.32 \cdot 10^{-3}$ & $2.59 \cdot 10^{-1}$ \\
35.00 & $2.44 \cdot 10^{-49}$ & $2.44 \cdot 10^{-49}$ & $1.32 \cdot 10^{-3}$ & $2.59 \cdot 10^{-1}$ \\
40.00 & $1.11 \cdot 10^{-41}$ & $1.11 \cdot 10^{-41}$ & $1.32 \cdot 10^{-3}$ & $2.59 \cdot 10^{-1}$ \\
45.00 & $1.01 \cdot 10^{-35}$ & $1.01 \cdot 10^{-35}$ & $1.32 \cdot 10^{-3}$ & $2.59 \cdot 10^{-1}$ \\
50.00 & $6.01 \cdot 10^{-31}$ & $6.01 \cdot 10^{-31}$ & $1.32 \cdot 10^{-3}$ & $2.59 \cdot 10^{-1}$ \\
200.00 & $1.93 \cdot 10^{2}$ & $1.93 \cdot 10^{2}$ & $1.05 \cdot 10^{3}$ & $2.21 \cdot 10^{3}$ \\
250.00 & $3.15 \cdot 10^{4}$ & $3.15 \cdot 10^{4}$ & $8.75 \cdot 10^{4}$ & $1.40 \cdot 10^{5}$ \\
300.00 & $9.58 \cdot 10^{5}$ & $9.58 \cdot 10^{5}$ & $1.87 \cdot 10^{6}$ & $2.63 \cdot 10^{6}$ \\
350.00 & $1.11 \cdot 10^{7}$ & $1.11 \cdot 10^{7}$ & $1.76 \cdot 10^{7}$ & $2.29 \cdot 10^{7}$ \\
\hline
\end{tabular}


Table S16: Rate constants of the reaction $\boldsymbol{d}_{\mathbf{1}} \mathbf{- 1 c} \rightarrow \boldsymbol{d}_{\mathbf{1}}-\mathbf{1 t}$ in $\mathrm{s}^{-1}$ computed at the B3LYP/6$311++G(3 d f, 3 p d)$ level of theory.

\begin{tabular}{lllll}
\hline$T / \mathrm{K}$ & $\mathrm{TST}$ & $\mathrm{CVT}$ & $\mathrm{CVT} / \mathrm{ZCT}$ & $\mathrm{CVT} / \mathrm{SCT}$ \\
\hline 0.10 & $\mathrm{NaN}$ & $\mathrm{NaN}$ & $\mathrm{NaN}$ & $\mathrm{NaN}$ \\
8.00 & $1.67 \cdot 10^{-257}$ & $1.67 \cdot 10^{-257}$ & $5.13 \cdot 10^{-9}$ & $3.13 \cdot 10^{-7}$ \\
0.00 & $9.58 \cdot 10^{-204}$ & $9.58 \cdot 10^{-204}$ & $5.14 \cdot 10^{-9}$ & $3.13 \cdot 10^{-7}$ \\
15.00 & $5.09 \cdot 10^{-132}$ & $5.09 \cdot 10^{-132}$ & $5.14 \cdot 10^{-9}$ & $3.14 \cdot 10^{-7}$ \\
20.00 & $4.04 \cdot 10^{-96}$ & $4.04 \cdot 10^{-96}$ & $5.15 \cdot 10^{-9}$ & $3.14 \cdot 10^{-7}$ \\
25.00 & $1.47 \cdot 10^{-74}$ & $1.47 \cdot 10^{-74}$ & $5.15 \cdot 10^{-9}$ & $3.14 \cdot 10^{-7}$ \\
30.00 & $3.61 \cdot 10^{-60}$ & $3.61 \cdot 10^{-60}$ & $5.15 \cdot 10^{-9}$ & $3.14 \cdot 10^{-7}$ \\
35.00 & $7.01 \cdot 10^{-50}$ & $7.01 \cdot 10^{-50}$ & $5.15 \cdot 10^{-9}$ & $3.14 \cdot 10^{-7}$ \\
40.00 & $3.71 \cdot 10^{-42}$ & $3.71 \cdot 10^{-42}$ & $5.15 \cdot 10^{-9}$ & $3.15 \cdot 10^{-7}$ \\
45.00 & $3.83 \cdot 10^{-36}$ & $3.83 \cdot 10^{-36}$ & $5.16 \cdot 10^{-9}$ & $3.16 \cdot 10^{-7}$ \\
50.00 & $2.51 \cdot 10^{-31}$ & $2.51 \cdot 10^{-31}$ & $5.20 \cdot 10^{-9}$ & $3.21 \cdot 10^{-7}$ \\
200.00 & $1.48 \cdot 10^{2}$ & $1.48 \cdot 10^{2}$ & $3.21 \cdot 10^{2}$ & $4.80 \cdot 10^{2}$ \\
250.00 & $2.47 \cdot 10^{4}$ & $2.47 \cdot 10^{4}$ & $3.74 \cdot 10^{4}$ & $4.89 \cdot 10^{4}$ \\
300.00 & $7.58 \cdot 10^{5}$ & $7.58 \cdot 10^{5}$ & $9.61 \cdot 10^{5}$ & $1.16 \cdot 10^{6}$ \\
350.00 & $8.84 \cdot 10^{6}$ & $8.84 \cdot 10^{6}$ & $1.02 \cdot 10^{7}$ & $1.17 \cdot 10^{7}$ \\
\hline
\end{tabular}

Table S17: Rate constants of the reaction $\mathbf{2 c} \rightarrow \mathbf{2 t}$ in $\mathrm{s}^{-1}$ computed at the B3LYP/6$311++G(3 d f, 3 p d)$ level of theory.

\begin{tabular}{lllll}
\hline$T / \mathrm{K}$ & TST & CVT & CVT/ZCT & CVT/SCT \\
\hline 0.10 & NaN & NaN & NaN & NaN \\
8.00 & $1.24 \cdot 10^{-268}$ & $1.24 \cdot 10^{-268}$ & $3.72 \cdot 10^{-6}$ & $7.39 \cdot 10^{-4}$ \\
10.00 & $1.20 \cdot 10^{-212}$ & $1.20 \cdot 10^{-212}$ & $3.72 \cdot 10^{-6}$ & $7.39 \cdot 10^{-4}$ \\
15.00 & $5.88 \cdot 10^{-138}$ & $5.88 \cdot 10^{-138}$ & $3.72 \cdot 10^{-6}$ & $7.39 \cdot 10^{-4}$ \\
20.00 & $1.42 \cdot 10^{-100}$ & $1.42 \cdot 10^{-100}$ & $3.72 \cdot 10^{-6}$ & $7.38 \cdot 10^{-4}$ \\
25.00 & $4.01 \cdot 10^{-78}$ & $4.01 \cdot 10^{-78}$ & $3.71 \cdot 10^{-6}$ & $7.37 \cdot 10^{-4}$ \\
30.00 & $3.84 \cdot 10^{-63}$ & $3.84 \cdot 10^{-63}$ & $3.71 \cdot 10^{-6}$ & $7.36 \cdot 10^{-4}$ \\
35.00 & $1.97 \cdot 10^{-52}$ & $1.97 \cdot 10^{-52}$ & $3.70 \cdot 10^{-6}$ & $7.34 \cdot 10^{-4}$ \\
40.00 & $2.17 \cdot 10^{-44}$ & $2.17 \cdot 10^{-44}$ & $3.69 \cdot 10^{-6}$ & $7.33 \cdot 10^{-4}$ \\
45.00 & $3.95 \cdot 10^{-38}$ & $3.95 \cdot 10^{-38}$ & $3.69 \cdot 10^{-6}$ & $7.32 \cdot 10^{-4}$ \\
50.00 & $4.07 \cdot 10^{-33}$ & $4.07 \cdot 10^{-33}$ & $3.68 \cdot 10^{-6}$ & $7.31 \cdot 10^{-4}$ \\
200.00 & $5.40 \cdot 10^{1}$ & $5.40 \cdot 10^{1}$ & $2.08 \cdot 10^{2}$ & $4.64 \cdot 10^{2}$ \\
250.00 & $1.14 \cdot 10^{4}$ & $1.14 \cdot 10^{4}$ & $2.19 \cdot 10^{4}$ & $3.94 \cdot 10^{4}$ \\
300.00 & $4.15 \cdot 10^{5}$ & $4.15 \cdot 10^{5}$ & $5.63 \cdot 10^{5}$ & $8.72 \cdot 10^{5}$ \\
350.00 & $5.49 \cdot 10^{6}$ & $5.49 \cdot 10^{6}$ & $6.23 \cdot 10^{6}$ & $8.62 \cdot 10^{6}$ \\
\hline
\end{tabular}


Table S18: Rate constants of the reaction $\boldsymbol{d}_{\mathbf{1}}-\mathbf{2 c} \rightarrow \boldsymbol{d}_{\mathbf{1}}-\mathbf{2 t}$ in $\mathrm{s}^{-1}$ computed at the B3LYP/6$311++G(3 d f, 3 p d)$ level of theory.

\begin{tabular}{lllll}
\hline$T / \mathrm{K}$ & $\mathrm{TST}$ & $\mathrm{CVT}$ & $\mathrm{CVT} / \mathrm{ZCT}$ & $\mathrm{CVT} / \mathrm{SCT}$ \\
\hline 0.10 & $\mathrm{NaN}$ & $\mathrm{NaN}$ & $\mathrm{NaN}$ & $\mathrm{NaN}$ \\
8.00 & $9.19 \cdot 10^{-276}$ & $9.20 \cdot 10^{-276}$ & $1.26 \cdot 10^{-13}$ & $3.13 \cdot 10^{-10}$ \\
10.00 & $2.36 \cdot 10^{-218}$ & $2.36 \cdot 10^{-218}$ & $1.27 \cdot 10^{-13}$ & $3.13 \cdot 10^{-10}$ \\
15.00 & $9.25 \cdot 10^{-142}$ & $9.26 \cdot 10^{-142}$ & $1.27 \cdot 10^{-13}$ & $3.14 \cdot 10^{-10}$ \\
20.00 & $1.99 \cdot 10^{-103}$ & $1.99 \cdot 10^{-103}$ & $1.27 \cdot 10^{-13}$ & $3.14 \cdot 10^{-10}$ \\
25.00 & $2.09 \cdot 10^{-80}$ & $2.10 \cdot 10^{-80}$ & $1.27 \cdot 10^{-13}$ & $3.14 \cdot 10^{-10}$ \\
30.00 & $4.82 \cdot 10^{-65}$ & $4.82 \cdot 10^{-65}$ & $1.26 \cdot 10^{-13}$ & $3.13 \cdot 10^{-10}$ \\
35.00 & $4.64 \cdot 10^{-54}$ & $4.64 \cdot 10^{-54}$ & $1.26 \cdot 10^{-13}$ & $3.13 \cdot 10^{-10}$ \\
40.00 & $8.15 \cdot 10^{-46}$ & $8.15 \cdot 10^{-46}$ & $1.26 \cdot 10^{-13}$ & $3.13 \cdot 10^{-10}$ \\
45.00 & $2.14 \cdot 10^{-39}$ & $2.14 \cdot 10^{-39}$ & $1.26 \cdot 10^{-13}$ & $3.12 \cdot 10^{-10}$ \\
50.00 & $2.95 \cdot 10^{-34}$ & $2.95 \cdot 10^{-34}$ & $1.26 \cdot 10^{-13}$ & $3.13 \cdot 10^{-10}$ \\
200.00 & $2.73 \cdot 10^{1}$ & $2.73 \cdot 10^{1}$ & $6.85 \cdot 10^{1}$ & $9.84 \cdot 10^{1}$ \\
250.00 & $6.47 \cdot 10^{3}$ & $6.47 \cdot 10^{3}$ & $1.13 \cdot 10^{4}$ & $1.50 \cdot 10^{4}$ \\
300.00 & $2.52 \cdot 10^{5}$ & $2.52 \cdot 10^{5}$ & $3.62 \cdot 10^{5}$ & $4.54 \cdot 10^{5}$ \\
350.00 & $3.49 \cdot 10^{6}$ & $3.49 \cdot 10^{6}$ & $4.45 \cdot 10^{6}$ & $5.35 \cdot 10^{6}$ \\
\hline
\end{tabular}

Table S19: Rate constants of the reaction $\mathbf{1 t} \rightarrow \mathbf{9}$ in $\mathrm{s}^{-1}$ computed at the B3LYP/6$311++G(3 d f, 3 p d)$ level of theory.

\begin{tabular}{lllll}
\hline$T / \mathrm{K}$ & $\mathrm{TST}$ & $\mathrm{CVT}$ & $\mathrm{CVT} / \mathrm{ZCT}$ & $\mathrm{CVT} / \mathrm{SCT}$ \\
\hline 0.10 & $\mathrm{NaN}$ & $\mathrm{NaN}$ & $\mathrm{NaN}$ & $\mathrm{NaN}$ \\
8.00 & 0.00 & 0.00 & $\mathrm{NaN}$ & $\mathrm{NaN}$ \\
10.00 & 0.00 & 0.00 & $\mathrm{NaN}$ & $\mathrm{NaN}$ \\
15.00 & 0.00 & 0.00 & $\mathrm{NaN}$ & $\mathrm{NaN}$ \\
20.00 & 0.00 & 0.00 & $\mathrm{NaN}$ & $\mathrm{NaN}$ \\
25.00 & $6.57 \cdot 10^{-285}$ & $6.47 \cdot 10^{-285}$ & $3.64 \cdot 10^{-9}$ & $4.78 \cdot 10^{-8}$ \\
30.00 & $1.63 \cdot 10^{-235}$ & $1.61 \cdot 10^{-235}$ & $3.64 \cdot 10^{-9}$ & $4.78 \cdot 10^{-8}$ \\
35.00 & $3.20 \cdot 10^{-200}$ & $3.17 \cdot 10^{-200}$ & $3.64 \cdot 10^{-9}$ & $4.78 \cdot 10^{-8}$ \\
40.00 & $9.60 \cdot 10^{-174}$ & $9.51 \cdot 10^{-174}$ & $3.64 \cdot 10^{-9}$ & $4.78 \cdot 10^{-8}$ \\
45.00 & $3.82 \cdot 10^{-153}$ & $3.79 \cdot 10^{-153}$ & $3.64 \cdot 10^{-9}$ & $4.78 \cdot 10^{-8}$ \\
50.00 & $1.16 \cdot 10^{-136}$ & $1.16 \cdot 10^{-136}$ & $3.64 \cdot 10^{-9}$ & $4.78 \cdot 10^{-8}$ \\
200.00 & $4.52 \cdot 10^{-25}$ & $4.51 \cdot 10^{-25}$ & $6.13 \cdot 10^{-9}$ & $6.94 \cdot 10^{-8}$ \\
250.00 & $1.47 \cdot 10^{-17}$ & $1.47 \cdot 10^{-17}$ & $1.85 \cdot 10^{-8}$ & $1.40 \cdot 10^{-7}$ \\
300.00 & $1.57 \cdot 10^{-12}$ & $1.57 \cdot 10^{-12}$ & $1.62 \cdot 10^{-7}$ & $6.47 \cdot 10^{-7}$ \\
350.00 & $6.35 \cdot 10^{-9}$ & $6.33 \cdot 10^{-9}$ & $3.51 \cdot 10^{-6}$ & $8.04 \cdot 10^{-6}$ \\
\hline
\end{tabular}


Table S20: Rate constants of the reaction $\boldsymbol{d}_{\mathbf{1}}-\mathbf{1 t} \rightarrow \boldsymbol{d}_{\mathbf{1}}-\mathbf{9}$ in $\mathrm{s}^{-1}$ computed at the B3LYP/6$311++G(3 d f, 3 p d)$ level of theory.

\begin{tabular}{lllll}
\hline$T / \mathrm{K}$ & $\mathrm{TST}$ & $\mathrm{CVT}$ & $\mathrm{CVT} / \mathrm{ZCT}$ & $\mathrm{CVT} / \mathrm{SCT}$ \\
\hline 0.10 & $\mathrm{NaN}$ & $\mathrm{NaN}$ & $\mathrm{NaN}$ & $\mathrm{NaN}$ \\
8.00 & 0.00 & 0.00 & $\mathrm{NaN}$ & $\mathrm{NaN}$ \\
10.00 & 0.00 & 0.00 & $\mathrm{NaN}$ & $\mathrm{NaN}$ \\
15.00 & 0.00 & 0.00 & $\mathrm{NaN}$ & $\mathrm{NaN}$ \\
20.00 & 0.00 & 0.00 & $\mathrm{NaN}$ & $\mathrm{NaN}$ \\
25.00 & $2.33 \cdot 10^{-294}$ & $2.33 \cdot 10^{-294}$ & $1.41 \cdot 10^{-17}$ & $6.05 \cdot 10^{-16}$ \\
30.00 & $2.18 \cdot 10^{-243}$ & $2.18 \cdot 10^{-243}$ & $1.41 \cdot 10^{-17}$ & $6.05 \cdot 10^{-16}$ \\
35.00 & $5.73 \cdot 10^{-207}$ & $5.73 \cdot 10^{-207}$ & $1.41 \cdot 10^{-17}$ & $6.05 \cdot 10^{-16}$ \\
40.00 & $1.20 \cdot 10^{-179}$ & $1.20 \cdot 10^{-179}$ & $1.41 \cdot 10^{-17}$ & $6.05 \cdot 10^{-16}$ \\
45.00 & $2.17 \cdot 10^{-158}$ & $2.17 \cdot 10^{-158}$ & $1.41 \cdot 10^{-17}$ & $6.05 \cdot 10^{-16}$ \\
50.00 & $2.22 \cdot 10^{-141}$ & $2.22 \cdot 10^{-141}$ & $1.41 \cdot 10^{-17}$ & $6.05 \cdot 10^{-16}$ \\
200.00 & $3.19 \cdot 10^{-26}$ & $3.19 \cdot 10^{-26}$ & $1.45 \cdot 10^{-16}$ & $2.49 \cdot 10^{-15}$ \\
250.00 & $1.80 \cdot 10^{-18}$ & $1.80 \cdot 10^{-18}$ & $1.98 \cdot 10^{-14}$ & $9.07 \cdot 10^{-14}$ \\
300.00 & $2.77 \cdot 10^{-13}$ & $2.77 \cdot 10^{-13}$ & $1.60 \cdot 10^{-11}$ & $2.88 \cdot 10^{-11}$ \\
350.00 & $1.44 \cdot 10^{-9}$ & $1.44 \cdot 10^{-9}$ & $1.32 \cdot 10^{-8}$ & $1.63 \cdot 10^{-8}$ \\
\hline
\end{tabular}




\section{NBO Analysis}

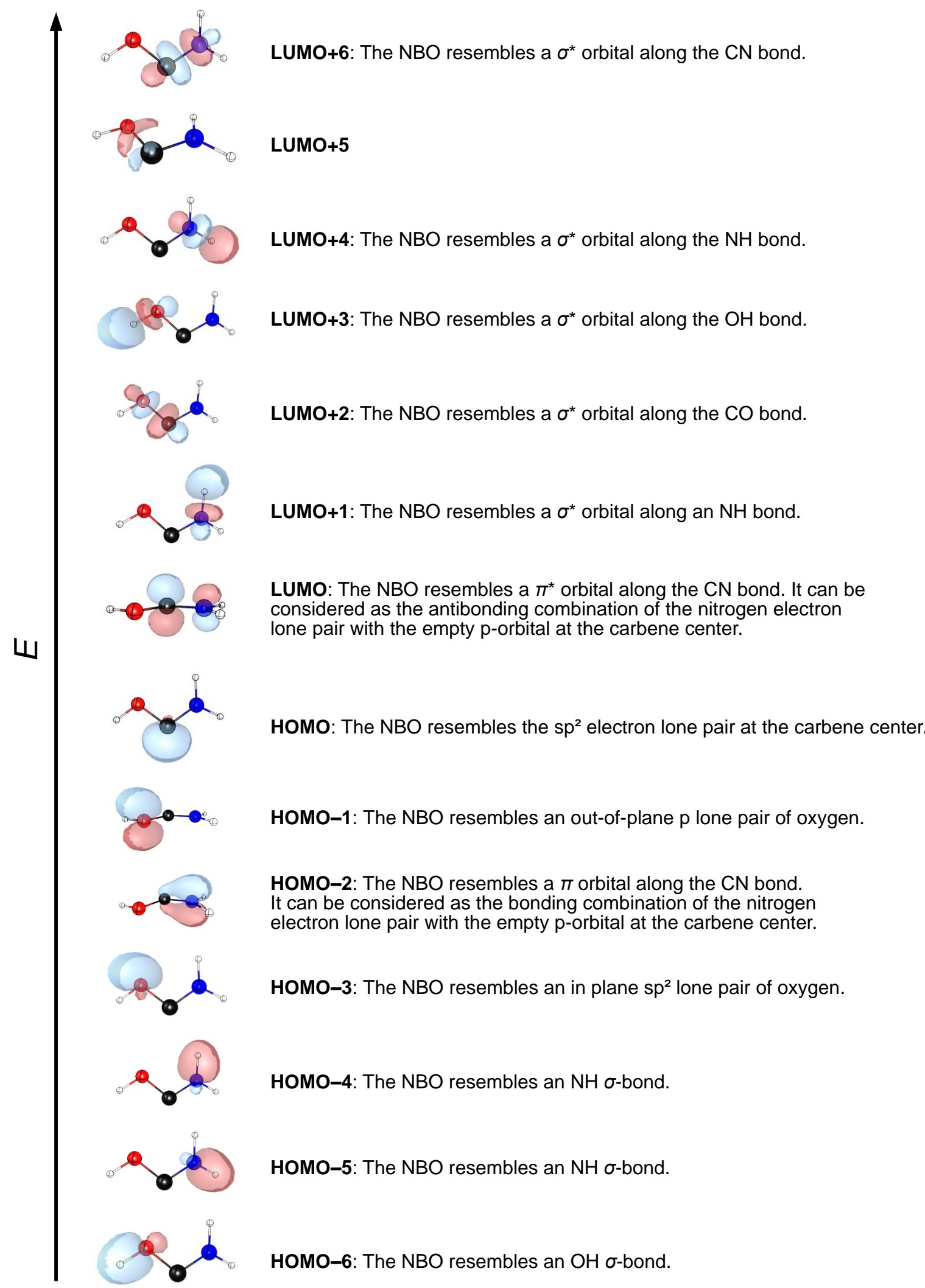




\section{Cartesian Coordinates of Computationally Optimized Geometries}

Optimized structures (Cartesian coordinates in Ångstrom), electronic energies, and zero-point vibrational energies (ZPVE) are provided. For transition states, the imaginary frequency is given additionally.

$B 3 L Y P / 6-311++G(3 d f, 3 p d)$

cis-Aminohydroxymethylene $1 \mathrm{c}\left(C_{s}\right)$

trans-Aminohydroxymethylene $\mathbf{1 t}\left(C_{\mathrm{s}}\right)$

$8 \quad-1.157566-0.036205 \quad 0.000000$

$8 \quad 1.083476-0.210474-0.000000$

$1-1.077520 \quad-1.015019 \quad 0.000000$

$1 \quad 1.857586 \quad 0.357331-0.000000$

$\begin{array}{llll}6 & -0.000000 & 0.612001 & 0.000000\end{array}$

$\begin{array}{llll}6 & -0.000000 & 0.590528 & -0.000000\end{array}$

$\begin{array}{llll}7 & 1.052325 & -0.194379 & -0.000000\end{array}$

$\begin{array}{llll}7 & -1.073850 & -0.181236 & 0.000000\end{array}$

$\begin{array}{llll}1 & 0.999157 & -1.216046 & -0.000000\end{array}$

$\begin{array}{llll}1 & -1.028302 & -1.197854 & 0.000000\end{array}$

$\begin{array}{llll}1 & 1.972621 & 0.209352 & -0.000000\end{array}$

$\begin{array}{llll}1 & -1.980144 & 0.249802 & 0.000000\end{array}$

$E=-169.8975099 \mathrm{au}$

$E=-169.9066293 \mathrm{au}$

ZPVE $=0.0444315 \mathrm{au}$

ZPVE $=0.0456975 \mathrm{au}$

cis-Aminohydroxymethylene- $\mathrm{CO}_{2}$ complex

$1 \mathrm{c}-\mathrm{CO}_{2}\left(C_{s}\right)$

trans-Aminohydroxymethylene- $\mathrm{CO}_{2}$

complex $1 \mathrm{t}-\mathrm{CO}_{2}\left(\mathrm{C}_{\mathrm{s}}\right)$

$\begin{array}{rrrr}6 & 0.000000 & 1.221154 & -0.000000 \\ 7 & 1.322823 & 1.222688 & -0.000000 \\ 8 & -0.540715 & 2.432243 & -0.000000 \\ 1 & 1.799520 & 0.335909 & 0.000000 \\ 1 & 1.902582 & 2.063892 & -0.000000 \\ 1 & 0.108508 & 3.167225 & -0.000000 \\ 6 & -0.401110 & -1.833537 & 0.000000 \\ 8 & 0.762238 & -1.828791 & 0.000000 \\ 8 & -1.554487 & -1.909895 & 0.000000\end{array}$

$\begin{array}{llll}6 & -0.000000 & 1.197212 & 0.000000\end{array}$

$\begin{array}{llll}7 & 1.316249 & 1.288611 & -0.000000\end{array}$

$\begin{array}{llll}8 & -0.515946 & 2.439311 & 0.000000\end{array}$

$11.853161 \quad 0.438810 \quad-0.000000$

$\begin{array}{lllll}1 & 1.812433 & 2.176076 & -0.000000\end{array}$

$\begin{array}{lllll}1 & -1.472499 & 2.354086 & 0.000000\end{array}$

$\begin{array}{llll}6 & -0.333837 & -1.820649 & 0.000000\end{array}$

$\begin{array}{lllll}8 & 0.828662 & -1.820319 & -0.000000\end{array}$

$\begin{array}{llll}8 & -1.488193 & -1.900070 & 0.000000\end{array}$

$E=-358.5629264 \mathrm{au}$

$E=-358.5720969 \mathrm{au}$

ZPVE $=0.0573163 \mathrm{au}$

ZPVE $=0.0584792 \mathrm{au}$ 
cis-Oxalic acid monoamide $2 \mathrm{c}\left(C_{s}\right)$

$\begin{array}{lrrr}8 & 1.066880 & 1.339909 & 0.000000 \\ 6 & -0.000000 & 0.749609 & 0.000000 \\ 6 & -0.010995 & -0.796866 & -0.000000 \\ 8 & 1.217833 & -1.295413 & -0.000000 \\ 8 & -1.016740 & -1.450215 & -0.000000 \\ 7 & -1.221338 & 1.296032 & 0.000000 \\ 1 & -2.027774 & 0.692822 & 0.000000 \\ 1 & -1.327839 & 2.295998 & 0.000000 \\ 1 & 1.827165 & -0.531759 & -0.000000 \\ & & \\ E=-358.6170602 \mathrm{au} & \\ Z P V E=0.0616047 \mathrm{au}\end{array}$

cis'-oxalic acid monoamide $\mathbf{2} \mathbf{c}^{\prime}\left(C_{s}\right)$

$\begin{array}{rrrr}7 & 1.297527 & 1.198891 & 0.000000 \\ 6 & -0.000000 & 0.775449 & 0.000000 \\ 8 & -0.930515 & 1.538302 & 0.000000 \\ 6 & -0.283957 & -0.762499 & -0.000000 \\ 8 & 0.760155 & -1.629821 & -0.000000 \\ 8 & -1.399588 & -1.168210 & -0.000000 \\ 1 & 1.619245 & -1.201318 & -0.000000 \\ 1 & 1.449624 & 2.194773 & 0.000000 \\ 1 & 2.111774 & 0.614437 & 0.000000\end{array}$

$E=-358.5889479 \mathrm{au}$

ZPVE $=0.0593135 \mathrm{au}$

$v_{\mathrm{i}}=361.5169 i \mathrm{~cm}^{-1}$

trans-Oxalic acid monoamide $2 \mathbf{t}\left(C_{s}\right)$

$\begin{array}{lrrr}8 & -1.090945 & 1.408119 & 0.000000 \\ 6 & -0.053054 & 0.789515 & 0.000000 \\ 6 & 0.000000 & -0.757767 & 0.000000 \\ 8 & -1.214059 & -1.301440 & 0.000000 \\ 8 & 1.031271 & -1.380139 & 0.000000 \\ 7 & 1.188846 & 1.318342 & 0.000000 \\ 1 & 1.993620 & 0.714885 & 0.000000 \\ 1 & 1.296640 & 2.317629 & 0.000000 \\ 1 & -1.103995 & -2.263723 & 0.000000 \\ & & \\ E= & -358.6104425 \mathrm{au} & \\ \text { ZPVE }=0.0610637 \mathrm{au} & \end{array}$

trans'-Oxalic acid monoamide $2 \mathbf{t}^{\prime}\left(C_{s}\right)$

$\begin{array}{lrrr}8 & -1.440149 & -1.133951 & 0.000000 \\ 6 & -0.000000 & 0.778800 & 0.000000 \\ 6 & -0.299537 & -0.742725 & 0.000000 \\ 7 & 0.798032 & -1.536155 & 0.000000 \\ 8 & 1.330356 & 1.040235 & 0.000000 \\ 8 & -0.842846 & 1.621265 & 0.000000 \\ 1 & 1.725918 & -1.152514 & 0.000000 \\ 1 & 0.667045 & -2.533731 & 0.000000 \\ 1 & 1.439148 & 2.002485 & 0.000000 \\ & & \\ E=-358.6065497 \mathrm{au} & \\ Z P V E=0.0606949 \mathrm{au} & \end{array}$

Formamide $9\left(C_{\mathrm{s}}\right)$

$\begin{array}{lrrr}6 & 0.000000 & 0.418768 & -0.000000 \\ 8 & -1.194948 & 0.234940 & -0.000000 \\ 7 & 0.935914 & -0.563447 & 0.000000 \\ 1 & 0.447925 & 1.427995 & -0.000000 \\ 1 & 0.643223 & -1.526956 & 0.000000 \\ 1 & 1.917042 & -0.349032 & 0.000000 \\ & & \\ \text { E }= & -169.9670722 \mathrm{au} \\ \text { ZPVE }=0.045276 \mathrm{au}\end{array}$

cis-trans-Formimidic acid 10ct $\left(C_{s}\right)$

$\begin{array}{lrrr}6 & 0.000000 & 0.418884 & -0.000000 \\ 7 & -1.230030 & 0.174482 & 0.000000 \\ 8 & 0.977185 & -0.529560 & 0.000000 \\ 1 & 0.361948 & 1.446102 & -0.000000 \\ 1 & -1.415519 & -0.828942 & 0.000000 \\ 1 & 1.846297 & -0.115356 & -0.000000 \\ & & \\ E & =-169.9414482 \mathrm{au} \\ \text { ZPVE }=0.045573 \mathrm{au}\end{array}$


trans-trans-Formimidic acid 10tt $\left(C_{s}\right)$

$$
\begin{array}{lrrc}
6 & 0.000000 & 0.439797 & -0.000000 \\
7 & 1.219555 & 0.128114 & 0.000000 \\
8 & -1.097614 & -0.356542 & -0.000000 \\
1 & -0.306319 & 1.481454 & -0.000000 \\
1 & 1.383636 & -0.879273 & 0.000000 \\
1 & -0.833290 & -1.285427 & -0.000000 \\
& & \\
\text { Z }= & -169.9417549 \mathrm{au} \\
\text { ZPVE }=0.0457111 \mathrm{au}
\end{array}
$$

trans-cis-Formimidic acid 10tc $\left(C_{s}\right)$

$$
\begin{array}{lrrr}
7 & 1.155799 & -0.085762 & 0.000000 \\
6 & 0.000000 & 0.415972 & 0.000000 \\
8 & -1.109857 & -0.341128 & 0.000000 \\
1 & 1.876855 & 0.627970 & 0.000000 \\
1 & -0.261108 & 1.473337 & 0.000000 \\
1 & -0.827478 & -1.267780 & 0.000000 \\
& & \\
E=-169.9474119 \mathrm{au} & \\
Z &
\end{array}
$$

cis-cis-Formimidic acid 10cc $\left(C_{\mathrm{s}}\right)$

$$
\begin{array}{lrll}
7 & 1.195476 & -0.009827 & 0.000000 \\
6 & 0.000000 & 0.374581 & 0.000000 \\
8 & -1.000844 & -0.535762 & 0.000000 \\
1 & 1.831004 & 0.781164 & 0.000000 \\
1 & -0.341027 & 1.414153 & 0.000000 \\
1 & -1.851558 & -0.087920 & 0.000000 \\
& & \\
E & =-169.9392491 \mathrm{au} \\
\text { ZPVE }=0.0456266 \mathrm{au}
\end{array}
$$

Aminohydroxymethane $11\left(C_{1}\right)$

$$
\begin{array}{rrrr}
6 & -0.039521 & 0.537131 & 0.015858 \\
8 & -1.219200 & -0.240268 & 0.053848 \\
1 & -1.123709 & -0.929439 & -0.612851 \\
7 & 1.131059 & -0.313652 & -0.052998 \\
1 & -0.093182 & 1.163777 & 0.910319 \\
1 & -0.023454 & 1.191110 & -0.862559 \\
1 & 1.381277 & -0.697389 & 0.849304 \\
1 & 1.932385 & 0.166867 & -0.439159
\end{array}
$$

$E=-171.1553841 \mathrm{au}$ ZPVE $=0.0692678 \mathrm{au}$

Oxalic acid monoamide - enolimine tautomer a $12 \mathrm{a}\left(C_{\mathrm{s}}\right)$

$$
\begin{array}{lrrr}
7 & -1.325192 & 1.294078 & -0.000000 \\
6 & -0.192748 & 0.743725 & -0.000000 \\
1 & -2.062781 & 0.593605 & 0.000000 \\
8 & 0.954913 & 1.439410 & -0.000000 \\
1 & 0.719234 & 2.377501 & -0.000000 \\
6 & -0.000000 & -0.764935 & 0.000000 \\
8 & -0.929488 & -1.525851 & 0.000000 \\
8 & 1.283655 & -1.138030 & 0.000000 \\
1 & 1.303734 & -2.106615 & 0.000000 \\
& & \\
E=-358.5883387 \text { au } & \\
\text { ZPVE }=0.0613291 \text { au } \\
\text { Tautomer b 12b }\left(C_{1}\right)
\end{array}
$$

$$
\begin{array}{lrrr}
7 & 1.460918 & 1.145546 & 0.000130 \\
6 & 0.735664 & 0.122382 & 0.000664 \\
1 & 0.887646 & 1.986657 & -0.000075 \\
6 & -0.791145 & 0.121066 & 0.000171 \\
8 & 1.246171 & -1.132278 & -0.000340 \\
8 & -1.415375 & 1.140461 & -0.000427 \\
8 & -1.368758 & -1.086211 & 0.000140 \\
1 & 2.211266 & -1.066795 & -0.001336 \\
1 & -0.688747 & -1.775151 & 0.000506 \\
& & \\
E= & -358.5881777 \mathrm{au} & \\
Z P V E=0.0612895 \mathrm{au} &
\end{array}
$$


Oxalic acid monoamide - enolimine tautomer c 12c $\left(C_{1}\right)$

$\begin{array}{lrrr}7 & 1.364678 & 1.254468 & -0.000227 \\ 1 & 0.820208 & -1.727535 & 0.000182 \\ 8 & 1.460326 & -0.995921 & 0.000251 \\ 6 & 0.766230 & 0.142035 & 0.000003 \\ 1 & 0.753385 & 2.063282 & -0.000435 \\ 6 & -0.745660 & -0.135067 & -0.000038 \\ 8 & -1.144262 & -1.268088 & -0.000271 \\ 8 & -1.583823 & 0.901592 & 0.000212 \\ 1 & -1.107690 & 1.740505 & 0.000512 \\ & & \\ E & =-358.5789172 \mathrm{au} & \\ Z P V E=0.0608522 \mathrm{au} & \end{array}$

Oxalic acid monoamide - enolimine tautomer $\mathrm{d} \mathbf{1 2 \mathrm { d }}\left(C_{1}\right)$

$\begin{array}{lrrc}7 & -1.446796 & 1.147061 & -0.242853 \\ 1 & -0.949608 & -1.582415 & 0.888367 \\ 8 & -1.366823 & -1.081982 & 0.177470 \\ 6 & -0.758725 & 0.131738 & 0.009941 \\ 1 & -0.840982 & 1.951719 & -0.393729 \\ 6 & 0.770304 & 0.127788 & 0.031828 \\ 8 & 1.413231 & 1.103240 & 0.276763 \\ 8 & 1.349049 & -1.054709 & -0.263932 \\ 1 & 0.685031 & -1.688278 & -0.567692 \\ & & \\ E= & -358.5712753 \mathrm{au} & \\ Z P V E=0.0605249 & \mathrm{au}\end{array}$

Oxalic acid monoamide - enolimine tautomer e $12 \mathrm{e}\left(C_{\mathrm{s}}\right)$

$$
\begin{array}{lrrr}
7 & -0.932260 & 1.546125 & 0.000000 \\
1 & 1.286762 & 2.162382 & 0.000000 \\
8 & 1.319861 & 1.195563 & 0.000000 \\
6 & 0.053838 & 0.759614 & 0.000000 \\
1 & -1.813540 & 1.043326 & 0.000000 \\
6 & 0.000000 & -0.760992 & 0.000000 \\
8 & 0.954115 & -1.481739 & 0.000000 \\
8 & -1.276376 & -1.195966 & 0.000000 \\
1 & -1.251229 & -2.163177 & 0.000000 \\
& & \\
E=-358.5866607 \mathrm{au} & \\
Z P V E=0.0612575 \mathrm{au} &
\end{array}
$$

Oxalic acid monoamide - enolimine tautomer $\mathrm{f} \mathbf{1 2 f}\left(C_{s}\right)$

$\begin{array}{lrrr}7 & -1.018353 & -1.542770 & -0.000000 \\ 1 & 1.863081 & -0.560934 & 0.000000 \\ 8 & 1.237111 & -1.301128 & 0.000000 \\ 6 & -0.003591 & -0.796629 & -0.000000 \\ 1 & -1.871969 & -0.993579 & -0.000000 \\ 6 & -0.000000 & 0.724389 & 0.000000 \\ 8 & 1.022739 & 1.361603 & 0.000000 \\ 8 & -1.223662 & 1.259984 & -0.000000 \\ 1 & -1.130598 & 2.223674 & -0.000000 \\ & & \\ E=-358.5875464 \mathrm{au} & \\ Z P V E=0.0615358 \mathrm{au}\end{array}$


Oxalic acid monoamide - enolimine tautomer g $12 \mathrm{~g}\left(C_{\mathrm{s}}\right)$

$$
\begin{array}{lrrr}
7 & -1.404606 & 1.254584 & 0.000000 \\
1 & 1.639988 & 1.010703 & -0.000000 \\
8 & 0.841036 & 1.550352 & 0.000000 \\
6 & -0.248356 & 0.760808 & 0.000000 \\
1 & -2.099045 & 0.511212 & 0.000000 \\
6 & 0.000000 & -0.742638 & -0.000000 \\
8 & -0.866091 & -1.568280 & -0.000000 \\
8 & 1.320130 & -1.034072 & -0.000000 \\
1 & 1.420833 & -1.997017 & -0.000000 \\
& & \\
E= & -358.5846074 \mathrm{au} & \\
Z P V E=0.0611949 \mathrm{au} &
\end{array}
$$

\begin{tabular}{|c|c|c|c|}
\hline 7 & -1.117005 & 1.330551 & 0.000000 \\
\hline 1 & 1.860529 & 0.572784 & -0.000000 \\
\hline & 1.210972 & 1.298290 & 0.000000 \\
\hline & -0.000000 & 0.741843 & 0.000000 \\
\hline & -1.043726 & 2.345159 & 0.000000 \\
\hline & 0.017792 & -0.779673 & -0.000000 \\
\hline & 1.059798 & -1.381719 & -0.000000 \\
\hline & -1.179958 & -1.340705 & -0.00000 \\
\hline & -1.831021 & -0.611748 & 0.00000 \\
\hline
\end{tabular}

Oxalic acid monoamide - enolimine tautomer h $\mathbf{1 2 h}\left(C_{1}\right)$

$$
\begin{array}{rrrc}
7 & -1.312442 & 1.152472 & -0.405116 \\
6 & -0.731336 & 0.094799 & -0.025531 \\
6 & 0.772747 & -0.168001 & -0.036530 \\
8 & 1.255780 & -1.180875 & -0.435360 \\
8 & -1.411421 & -0.971457 & 0.402753 \\
8 & 1.529143 & 0.865842 & 0.390581 \\
1 & -0.673005 & 1.864692 & -0.743260 \\
1 & -2.355051 & -0.767302 & 0.325710 \\
1 & 0.978661 & 1.566433 & 0.761935
\end{array}
$$

$E=-358.5775149 \mathrm{au}$ ZPVE $=0.0609304 \mathrm{au}$
Oxalic acid monoamide - enolimine tautomer i 12i $\left(C_{1}\right)$

$\begin{array}{lrrr}7 & -1.389842 & 1.133518 & -0.321850 \\ 1 & -2.181248 & -1.141256 & 0.285833 \\ 8 & -1.216322 & -1.137199 & 0.311924 \\ 6 & -0.752983 & 0.093047 & -0.013144 \\ 1 & -2.400718 & 1.004456 & -0.330873 \\ 6 & 0.767938 & 0.148741 & 0.028013 \\ 8 & 1.393422 & 1.119754 & 0.336482 \\ 8 & 1.315909 & -1.024652 & -0.335001 \\ 1 & 2.277062 & -0.911772 & -0.298457 \\ & & \\ E & =-358.5771032 \mathrm{au} & \\ \text { ZPVE }=0.0605886 \mathrm{au} & \end{array}$

Oxalic acid monoamide - enolimine tautomer j $\mathbf{1 2} \mathbf{j}\left(C_{s}\right)$ 
Oxalic acid monoamide - enolimine tautomer $\mathrm{k} \mathbf{1 2 k}\left(C_{1}\right)$

$\begin{array}{lrrr}7 & 1.169771 & 1.284257 & -0.000330 \\ 1 & 0.815191 & -1.767584 & -0.000233 \\ 8 & 1.447831 & -1.027411 & 0.000544 \\ 6 & 0.735145 & 0.098664 & -0.000406 \\ 1 & 2.185185 & 1.346405 & 0.000134 \\ 6 & -0.770382 & -0.121452 & -0.000264 \\ 8 & -1.228733 & -1.234378 & -0.000242 \\ 8 & -1.485164 & 0.991168 & 0.000414 \\ 1 & -0.848826 & 1.733065 & 0.000706 \\ & & \\ \text { E }=-358.5957761 \mathrm{au} & \\ \text { ZPVE }=0.0617951 \mathrm{au} & \end{array}$

Oxalic acid monoamide - enolimine tautomer I 12I $\left(C_{s}\right)$

$$
\begin{array}{lrrr}
7 & -1.085408 & 1.392471 & 0.000000 \\
1 & 1.259598 & 2.179978 & 0.000000 \\
8 & 1.267586 & 1.215632 & 0.000000 \\
6 & -0.000000 & 0.750919 & 0.000000 \\
1 & -0.958444 & 2.404396 & 0.000000 \\
6 & 0.007977 & -0.771892 & 0.000000 \\
8 & 1.010414 & -1.429601 & 0.000000 \\
8 & -1.230083 & -1.281212 & 0.000000 \\
1 & -1.134489 & -2.244391 & 0.000000 \\
& & \\
E=-358.5783534 \mathrm{au} & \\
Z P V E=0.0607015 \mathrm{au} & \\
v_{\mathrm{i}}=5.1956 i \mathrm{~cm}^{-1} &
\end{array}
$$

Oxalic acid monoamide - enolimine tautomer $\mathrm{m} 12 \mathrm{~m}\left(C_{1}\right)$

$$
\begin{array}{rrrc}
7 & 1.386872 & 1.200246 & 0.000049 \\
1 & 2.152199 & -1.177551 & -0.000706 \\
8 & 1.188140 & -1.156135 & 0.000102 \\
6 & 0.727507 & 0.133680 & -0.000086 \\
1 & 2.396890 & 1.066137 & 0.000134 \\
6 & -0.806108 & 0.159903 & -0.000010 \\
8 & -1.434077 & 1.170768 & -0.000017 \\
8 & -1.388011 & -1.052852 & 0.000004 \\
1 & -0.714001 & -1.746053 & 0.000096
\end{array}
$$

$E=-358.5753888 \mathrm{au}$ ZPVE $=0.0605126 \mathrm{au}$

Oxalic acid monoamide - enolimine tautomer $\mathrm{n} \mathbf{1 2 n}\left(C_{\mathrm{s}}\right)$

$$
\begin{array}{lrrr}
7 & -1.138003 & 1.403792 & 0.000000 \\
1 & 1.828086 & 0.590532 & 0.000000 \\
8 & 1.182811 & 1.317109 & 0.000000 \\
6 & -0.048331 & 0.773823 & 0.000000 \\
1 & -0.982127 & 2.411451 & 0.000000 \\
6 & -0.000000 & -0.741716 & -0.000000 \\
8 & 1.059824 & -1.323375 & -0.000000 \\
8 & -1.186826 & -1.334910 & -0.000000 \\
1 & -1.036424 & -2.291766 & -0.000000 \\
& & \\
E=-358.5887231 \mathrm{au} & \\
Z P V E=0.0613822 \mathrm{au} &
\end{array}
$$


Oxalic acid monoamide - enolimine tautomer o $120\left(C_{1}\right)$

$\begin{array}{rrrr}7 & 1.469902 & -1.133300 & -0.000705 \\ 1 & 0.554690 & 1.815272 & 0.001463 \\ 8 & 1.275136 & 1.172134 & 0.000448 \\ 6 & 0.775333 & -0.085893 & 0.000351 \\ 1 & 2.465484 & -0.914537 & -0.000982 \\ 6 & -0.742133 & -0.205507 & 0.000141 \\ 8 & -1.367036 & -1.219628 & 0.000608 \\ 8 & -1.312564 & 1.030820 & -0.000760 \\ 1 & -2.272968 & 0.914163 & -0.000864\end{array}$

$E=-358.5825086 \mathrm{au}$

ZPVE $=0.0608502 \mathrm{au}$

Oxalic acid monoamide - enolimine tautomer $p 12 p\left(C_{s}\right)$

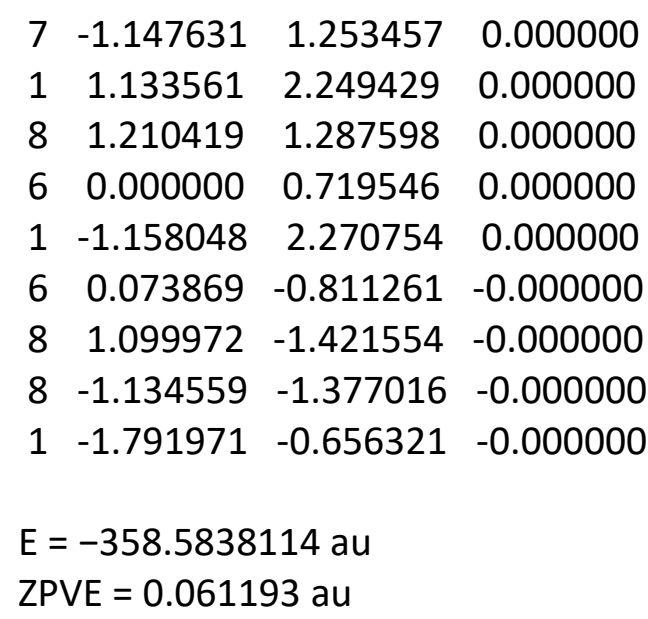

TS1: 1c $\rightarrow$ 10tt $\left(C_{1}\right)$

$\begin{array}{llll}8 & -1.159426 & 0.062153 & 0.023102\end{array}$

$\begin{array}{llll}1 & -1.077934 & 1.038211 & 0.062813\end{array}$

$\begin{array}{llll}6 & 0.028204 & -0.512642 & -0.095280\end{array}$

$\begin{array}{llll}7 & 1.151394 & 0.180385 & -0.064084\end{array}$

$\begin{array}{llll}1 & 1.078191 & 1.185798 & 0.197131\end{array}$

$\begin{array}{lllll}1 & 1.046166 & -0.908080 & 0.575504\end{array}$

$E=-169.8215473 \mathrm{au}$

ZPVE $=0.0385016 \mathrm{au}$

$v_{\mathrm{i}}=1827.1419 i \mathrm{~cm}^{-1}$
TS2: $1 \mathrm{c} \rightarrow \mathrm{HNCO}+\mathrm{H}_{2}\left(C_{s}\right)$

$\begin{array}{lrrr}8 & -1.117700 & 0.080055 & 0.000000 \\ 1 & -0.664569 & -1.345965 & -0.000000 \\ 6 & -0.000000 & 0.550826 & 0.000000 \\ 7 & 1.028336 & -0.186119 & -0.000000 \\ 1 & 0.399069 & -1.341278 & -0.000000 \\ 1 & 2.008748 & 0.044682 & -0.000000 \\ & & \\ E= & -169.828023 \mathrm{au} \\ Z P V E= & 0.034107 \mathrm{au}^{-1}\end{array}$

TS3: $1 \mathrm{c} \rightarrow \mathrm{NH}_{3}+\mathrm{CO}\left(C_{1}\right)$

$\begin{array}{lrrc}8 & 1.078253 & -0.273108 & -0.000000 \\ 1 & 0.043994 & -0.955884 & -0.000001 \\ 6 & 0.296689 & 0.707596 & 0.000000 \\ 7 & -1.033389 & -0.158691 & -0.000000 \\ 1 & -1.608210 & 0.003001 & 0.824890 \\ 1 & -1.608211 & 0.003002 & -0.824890 \\ & & \\ E= & -169.8332824 \mathrm{au} \\ Z P V E=0.0387591 \mathrm{au} & \\ v_{\mathrm{i}}=1753.1746 i \mathrm{~cm}^{-1} & \end{array}$

TS4: (valley ridge inflection point) $1 \mathrm{c} \rightarrow$ $\mathrm{NH}_{3}+\mathrm{CO}\left(C_{1}\right)$

$$
\begin{array}{llll}
8 & 1.149458 & -0.124688 & -0.000000 \\
1 & 0.881812 & -1.077181 & -0.000000 \\
6 & 0.098351 & 0.624930 & 0.000000 \\
7 & -1.058200 & -0.250434 & -0.000000 \\
1 & -1.630089 & 0.039071 & 0.794738 \\
1 & -1.630089 & 0.039073 & -0.794737 \\
& & \\
E=-169.863114 \mathrm{au} & \\
Z P V E=0.0432627 \mathrm{au} & \\
V_{\mathrm{i}}=451.7374 i \mathrm{~cm}^{-1} &
\end{array}
$$


TS5: $1 \mathrm{c} \rightarrow$ 1t $\left(C_{1}\right)$

$$
\begin{array}{cccc}
8 & 1.134372 & 0.134103 & -0.110618 \\
1 & 1.405635 & 0.510609 & 0.735098 \\
6 & -0.016077 & -0.625231 & 0.030198 \\
7 & -1.059977 & 0.173197 & 0.004017 \\
1 & -0.982196 & 1.184211 & -0.114558 \\
1 & -1.982117 & -0.228644 & 0.055103
\end{array}
$$

$E=-169.8809536 \mathrm{au}$

ZPVE $=0.0433915 \mathrm{au}$

$v_{\mathrm{i}}=696.8516 i \mathrm{~cm}^{-1}$

$$
\text { TS6: 1t } \rightarrow \mathrm{HNC}+\mathrm{H}_{2} \mathrm{O}\left(C_{1}\right)
$$

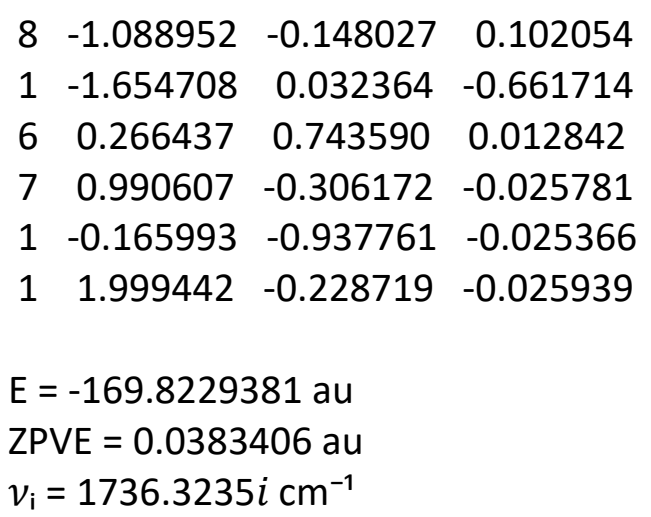

$$
\text { TS7: 1t } \rightarrow \text { 10ct }\left(C_{1}\right)
$$

$$
\begin{array}{lrrr}
8 & 1.084198 & -0.248123 & 0.036816 \\
1 & 1.849156 & 0.334820 & -0.015447 \\
6 & -0.029086 & 0.490561 & -0.108051 \\
7 & -1.178048 & -0.152468 & -0.061100 \\
1 & -1.110428 & -1.154069 & 0.196229 \\
1 & -0.991458 & 0.928144 & 0.600699 \\
& & \\
E=-169.8272805 \mathrm{au} & \\
Z P V E=0.0393711 \mathrm{au} & \\
v_{\mathrm{i}}=1808.2460 i \mathrm{~cm}^{-1} &
\end{array}
$$

TS8: $1 \mathrm{t} \rightarrow \mathbf{9}\left(C_{1}\right)$

$$
\begin{array}{lrrr}
8 & -1.149493 & 0.288832 & 0.000000 \\
6 & -0.079672 & -0.470537 & 0.000000 \\
7 & 1.110488 & 0.107627 & 0.000000 \\
1 & 1.175000 & 1.122397 & -0.000000 \\
1 & 1.952453 & -0.439341 & -0.000000 \\
1 & -1.226895 & -0.923874 & -0.000000 \\
& & \\
E= & -169.8463348 \mathrm{au} \\
\text { ZPVE }=0.0393411 \mathrm{au} & \\
v_{\mathrm{i}}=1980.1578 i \mathrm{~cm}^{-1}
\end{array}
$$

TS9: $1 \mathrm{t}-\mathrm{CO}_{2} \rightarrow 2 \mathrm{c}\left(C_{\mathrm{s}}\right)$

$$
\begin{array}{lrrr}
6 & 0.000000 & 0.888571 & 0.000000 \\
7 & 0.714564 & 1.992199 & -0.000000 \\
8 & -1.294548 & 1.122071 & 0.000000 \\
1 & 1.716959 & 1.921098 & -0.000000 \\
1 & 0.299802 & 2.919054 & -0.000000 \\
1 & -1.716089 & 0.243154 & 0.000000 \\
6 & 0.236848 & -1.348777 & -0.000000 \\
8 & 1.382935 & -1.590176 & -0.000000 \\
8 & -0.928851 & -1.565328 & 0.000000 \\
& & \\
E & =-358.5699649 \mathrm{au} & \\
Z \mathrm{ZPVE}=0.0589707 \mathrm{au} & \\
v_{\mathrm{i}}=174.7086 i \mathrm{~cm}^{-1}
\end{array}
$$

$$
\begin{aligned}
& \text { TS10: } 1 \mathrm{c}-\mathrm{CO}_{2} \rightarrow 1 \mathrm{t}-\mathrm{CO}_{2}\left(C_{1}\right) \\
& \begin{array}{llll}
6 & 1.185716 & -0.215527 & -0.009939
\end{array} \\
& \begin{array}{lllll}
7 & 1.433737 & 1.072265 & -0.000462
\end{array} \\
& \begin{array}{llll}
8 & 2.373316 & -0.918753 & -0.095032
\end{array} \\
& \begin{array}{llll}
1 & 0.652702 & 1.710641 & 0.016207
\end{array} \\
& \begin{array}{lllll}
1 & 2.376811 & 1.456375 & -0.057275
\end{array} \\
& \begin{array}{llll}
1 & 2.783464 & -1.016768 & 0.772415
\end{array} \\
& \begin{array}{llll}
6 & -1.880591 & -0.054676 & 0.003436
\end{array} \\
& \begin{array}{llll}
8 & -1.666010 & 1.088878 & 0.012034
\end{array} \\
& \begin{array}{lllll}
8 & -2.167292 & -1.174485 & -0.003138
\end{array} \\
& E=-358.5464388 \mathrm{au} \\
& \text { ZPVE }=0.0562246 \mathrm{au} \\
& v_{\mathrm{i}}=696.8683 i \mathrm{~cm}^{-1}
\end{aligned}
$$


TS11: $2 \mathrm{t} \rightarrow 2 \mathrm{c}\left(C_{1}\right)$

$\begin{array}{rrrr}8 & 1.051522 & 1.401683 & 0.130580 \\ 6 & -0.741892 & -0.214357 & 0.001536 \\ 6 & 0.741394 & 0.239206 & 0.003239 \\ 7 & 1.593194 & -0.800785 & -0.117797 \\ 8 & -1.620727 & 0.788926 & -0.228575 \\ 8 & -1.055070 & -1.359103 & 0.150555 \\ 1 & 1.239103 & -1.742041 & -0.130898 \\ 1 & 2.583323 & -0.631939 & -0.070528 \\ 1 & -1.977598 & 1.178336 & 0.576872\end{array}$

$E=-358.5922733 \mathrm{au}$

ZPVE $=0.0591957 \mathrm{au}$

$v_{\mathrm{i}}=653.9088 i \mathrm{~cm}^{-1}$

TS12: $12 \mathrm{~b} \rightarrow 2 \mathrm{c}\left(C_{\mathrm{s}}\right)$

$\begin{array}{llll}7 & -0.893497 & 1.603760 & 0.000000\end{array}$

$\begin{array}{llll}6 & -0.000000 & 0.674197 & 0.000000\end{array}$

$\begin{array}{llll}1 & -1.890859 & 1.438718 & 0.000000\end{array}$

$\begin{array}{llll}6 & -0.178176 & -0.830751 & 0.000000\end{array}$

$\begin{array}{llll}8 & 1.172199 & 1.215592 & 0.000000\end{array}$

$\begin{array}{llll}8 & -1.252251 & -1.354375 & 0.000000\end{array}$

$\begin{array}{lllll}8 & 0.978883 & -1.496423 & 0.000000\end{array}$

$\begin{array}{llll}1 & 0.312519 & 2.214488 & 0.000000\end{array}$

$11.711216 \quad-0.858551 \quad 0.000000$

$E=-358.531635 \mathrm{au}$

ZPVE $=0.0559131 \mathrm{au}$

$v_{\mathrm{i}}=1988.9847 i \mathrm{~cm}^{-1}$
TS13: $12 \mathrm{a} \rightarrow 2 \mathrm{t}\left(C_{\mathrm{s}}\right)$

$\begin{array}{llll}7 & -1.011588 & 1.514720 & -0.000000\end{array}$

$\begin{array}{llll}6 & 0.000000 & 0.703790 & 0.000000\end{array}$

$\begin{array}{lllll}1 & -1.980145 & 1.224974 & -0.000000\end{array}$

$\begin{array}{llll}8 & 1.086010 & 1.380613 & 0.000000\end{array}$

$\begin{array}{llll}6 & -0.087597 & -0.806272 & -0.000000\end{array}$

$\begin{array}{lllll}8 & -1.136838 & -1.389699 & -0.000000\end{array}$

$\begin{array}{llll}8 & 1.114657 & -1.383328 & 0.000000\end{array}$

$\begin{array}{llll}1 & 0.981148 & -2.343104 & 0.000000\end{array}$

$\begin{array}{llll}1 & 0.095061 & 2.269299 & 0.000000\end{array}$

$E=-358.5295194 \mathrm{au}$ ZPVE $=0.0557261 \mathrm{au}$ $v_{\mathrm{i}}=1989.5143 i \mathrm{~cm}^{-1}$

TS14: 10 tc $\rightarrow 9\left(C_{s}\right)$

$\begin{array}{lrrr}7 & 0.987940 & -0.332840 & 0.000000 \\ 6 & 0.000000 & 0.508905 & 0.000000 \\ 8 & -1.098812 & -0.145558 & 0.000000 \\ 1 & 1.968804 & -0.090442 & 0.000000 \\ 1 & 0.040022 & 1.594790 & 0.000000 \\ 1 & -0.133903 & -1.063436 & 0.000000 \\ & & \\ E=-169.8909763 \mathrm{au} & \\ Z P V E=0.0403924 \mathrm{au} & \\ v_{\mathrm{i}}=1942.6154 i \mathrm{~cm}^{-1} & \end{array}$

TS15: $10 \mathrm{cc} \rightarrow 10 \mathrm{tc}\left(C_{1}\right)$

$\begin{array}{llll}7 & 1.132509 & -0.347000 & 0.003332\end{array}$

$\begin{array}{llll}6 & 0.111121 & 0.381099 & 0.008225\end{array}$

$\begin{array}{llll}8 & -1.142089 & -0.156415 & -0.113347\end{array}$

$\begin{array}{lllll}1 & 1.976139 & 0.221016 & 0.042584\end{array}$

$\begin{array}{llll}1 & 0.111774 & 1.474299 & 0.043220\end{array}$

$\begin{array}{llll}1 & -1.545494 & -0.301589 & 0.748302\end{array}$

$E=-169.9300091 \mathrm{au}$

ZPVE $=0.044304 \mathrm{au}$

$v_{\mathrm{i}}=536.9402 i \mathrm{~cm}^{-1}$ 
TS16: 10tt $\rightarrow$ 10tc $\left(C_{s}\right)$

$\begin{array}{llll}7 & 1.146539 & -0.161745 & 0.000001\end{array}$

$\begin{array}{llll}6 & 0.074299 & 0.425919 & 0.000000\end{array}$

$\begin{array}{llll}8 & -1.169509 & -0.146349 & -0.000001\end{array}$

$\begin{array}{llll}1 & 2.000130 & -0.658328 & 0.000002\end{array}$

$\begin{array}{llll}1 & -0.068072 & 1.511886 & -0.000001\end{array}$

$\begin{array}{llll}1 & -1.047554 & -1.106063 & -0.000001\end{array}$

$E=-169.9108543 \mathrm{au}$

ZPVE $=0.0429051 \mathrm{au}$

$v_{\mathrm{i}}=1033.5837 i \mathrm{~cm}^{-1}$

TS17: $10 \mathrm{cc} \rightarrow 10 \mathrm{ct}\left(C_{\mathrm{s}}\right)$

$\begin{array}{llll}7 & 1.178528 & 0.096690 & 0.000000\end{array}$

$\begin{array}{llll}6 & 0.000000 & 0.396002 & 0.000000\end{array}$

$\begin{array}{lllll}8 & -1.015421 & -0.534572 & 0.000000\end{array}$

$12.140988 \quad-0.121942 \quad 0.000000$

$\begin{array}{llll}1 & -0.403441 & 1.421840 & 0.000000\end{array}$

$\begin{array}{llll}1 & -1.863874 & -0.076158 & 0.000000\end{array}$

$E=-169.9059576 \mathrm{au}$

ZPVE $=0.0426447 \mathrm{au}$

$v_{\mathrm{i}}=1022.3009 i \mathrm{~cm}^{-1}$

TS18: 10ct $\rightarrow$ 10tt $\left(C_{1}\right)$
TS19: $\mathrm{HNC} \rightarrow \mathrm{HCN}\left(C_{s}\right)$

$\begin{array}{llll}7 & 0.080706 & -0.563594 & 0.000000\end{array}$

$\begin{array}{lllll}6 & 0.080706 & 0.616306 & 0.000000\end{array}$

$\begin{array}{llll}1 & -1.049175 & 0.247322 & 0.000000\end{array}$

$E=-93.3852009 \mathrm{au}$

ZPVE $=0.0106307 \mathrm{au}$

$v_{\mathrm{i}}=1126.0690 i \mathrm{~cm}^{-1}$

\footnotetext{
$\begin{array}{llll}7 & 1.224256 & -0.153989 & 0.012607\end{array}$

$\begin{array}{lllll}6 & 0.115257 & 0.429257 & 0.014457\end{array}$

$\begin{array}{lllll}8 & -1.121767 & -0.174305 & -0.114500\end{array}$

$\begin{array}{lllll}1 & 1.111253 & -1.166471 & -0.057810\end{array}$

$\begin{array}{llll}1 & 0.062248 & 1.515457 & 0.056136\end{array}$

$\begin{array}{llll}1 & -1.460699 & -0.452163 & 0.742679\end{array}$

$E=-169.9310836 \mathrm{au}$

ZPVE $=0.0443124 \mathrm{au}$

$v_{\mathrm{i}}=494.562 i \mathrm{~cm}^{-1}$
} 
cis-Aminohydroxymethylene $1 \mathrm{c}\left(C_{\mathrm{s}}\right)$

$\begin{array}{llll}8 & -1.11232 & -0.14393 & 0.00000\end{array}$

$\begin{array}{llll}1 & -0.93795 & -1.10520 & 0.00000\end{array}$

$\begin{array}{llll}6 & 0.01246 & 0.58206 & 0.00000\end{array}$

$\begin{array}{lllll}7 & 1.10455 & -0.18676 & 0.00000\end{array}$

$\begin{array}{llll}1 & 1.09253 & -1.20548 & 0.00000\end{array}$

$\begin{array}{llll}1 & 2.00342 & 0.25931 & 0.00000\end{array}$

$E=-169.5743490001 \mathrm{au}$

ZPVE $=0.0448864840 \mathrm{au}$

cis-Aminohydroxymethylene- $\mathrm{CO}_{2}$ complex $1 \mathrm{c}-\mathrm{CO}_{2}\left(C_{\mathrm{s}}\right)$

$E=-357.9089574872 \mathrm{au}$

ZPVE $=0.0576926624 \mathrm{au}$

trans-Aminohydroxymethylene 1t $\left(C_{s}\right)$

$\begin{array}{llll}8 & -1.05431 & 0.24187 & 0.00000\end{array}$

$\begin{array}{llll}1 & -1.82463 & -0.32997 & 0.00000\end{array}$

$\begin{array}{llll}6 & 0.01814 & -0.58932 & 0.00000\end{array}$

$\begin{array}{llll}7 & 1.10042 & 0.18435 & 0.00000\end{array}$

$\begin{array}{llll}1 & 1.04827 & 1.19697 & 0.00000\end{array}$

$\begin{array}{llll}1 & 2.00343 & -0.25013 & 0.00000\end{array}$

$E=-169.5837140548 \mathrm{au}$

ZPVE $=0.0459565872 \mathrm{au}$

trans-Aminohydroxymethylene- $\mathrm{CO}_{2}$ complex $1 \mathrm{t}-\mathrm{CO}_{2}\left(C_{\mathrm{s}}\right)$
$\begin{array}{llll}6 & 1.21552 & 0.18543 & 0.00000\end{array}$
$\begin{array}{lllll}7 & 1.47673 & -1.11540 & 0.00000\end{array}$
$\begin{array}{llll}8 & 2.40154 & 0.83887 & 0.00000\end{array}$
$\begin{array}{lllll}1 & 0.70207 & -1.75384 & 0.00000\end{array}$
$12.42089-1.48396 \quad 0.00000$
$\begin{array}{llll}1 & 2.18520 & 1.77401 & 0.00000\end{array}$
$\begin{array}{llll}6 & -1.77114 & 0.04032 & 0.00000\end{array}$
$\begin{array}{llll}8 & -1.57905 & -1.11357 & 0.00000\end{array}$
$\begin{array}{llll}8 & -2.03293 & 1.17406 & 0.00000\end{array}$

$E=-357.9184698404 \mathrm{au}$ ZPVE $=0.0587085831 \mathrm{au}$

cis-Oxalic acid monoamide $\mathbf{2 c}\left(C_{\mathrm{s}}\right)$

$\begin{array}{llll}8 & -1.21491 & 1.22764 & 0.00000\end{array}$

$\begin{array}{llll}6 & -0.76916 & 0.08714 & 0.00000\end{array}$

$\begin{array}{llll}6 & 0.75522 & -0.12948 & 0.00000\end{array}$

$\begin{array}{llll}7 & -1.46550 & -1.05881 & 0.00000\end{array}$

$\begin{array}{llll}8 & 1.40949 & 1.02948 & 0.00000\end{array}$

$\begin{array}{llll}8 & 1.27027 & -1.21902 & 0.00000\end{array}$

$\begin{array}{llll}1 & -0.95683 & -1.92650 & 0.00000\end{array}$

$\begin{array}{llll}1 & -2.46949 & -1.04091 & 0.00000\end{array}$

$\begin{array}{lllll}1 & 0.70629 & 1.70769 & 0.00000\end{array}$

$E=-357.9647865001 \mathrm{au}$ ZPVE $=0.0619644696 \mathrm{au}$ 


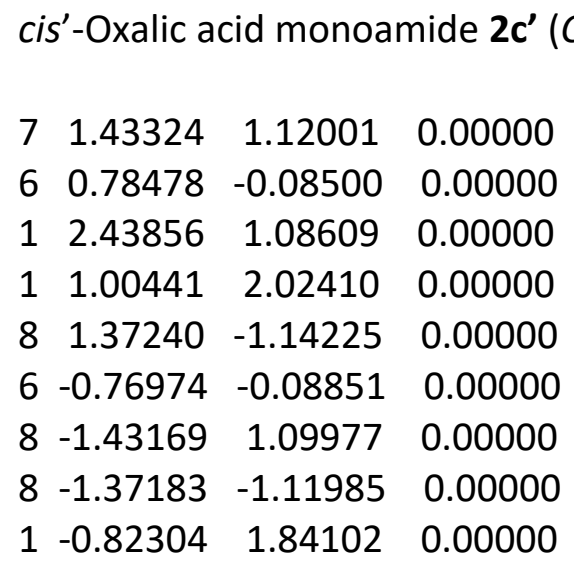

$E=-357.9358480003 \mathrm{au}$

ZPVE $=0.0595329530 \mathrm{au}$

trans-Oxalic acid monoamide $\mathbf{2 t}\left(C_{\mathrm{s}}\right)$

$\begin{array}{rrrr}8 & -1.28419 & 1.22812 & 0.00000 \\ 6 & -0.78700 & 0.12102 & 0.00000 \\ 6 & 0.73591 & -0.10944 & 0.00000 \\ 7 & -1.44828 & -1.05955 & 0.00000 \\ 8 & 1.41657 & 1.03901 & 0.00000 \\ 8 & 1.23842 & -1.21060 & 0.00000 \\ 1 & -0.92536 & -1.91707 & 0.00000 \\ 1 & -2.45197 & -1.05958 & 0.00000 \\ 1 & 2.35297 & 0.79246 & 0.00000\end{array}$

$E=-357.9580695064 \mathrm{au}$

ZPVE $=0.0612495801 \mathrm{au}$

trans'-Oxalic acid monoamide $2 \mathrm{t}^{\prime}\left(C_{\mathrm{s}}\right)$

$\begin{array}{rrrr}8 & -1.42043 & 1.13139 & 0.00000 \\ 6 & -0.79537 & 0.09324 & 0.00000 \\ 6 & 0.74710 & 0.11633 & 0.00000 \\ 7 & -1.33491 & -1.15209 & 0.00000 \\ 8 & 1.27850 & -1.13506 & 0.00000 \\ 8 & 1.40044 & 1.12115 & 0.00000 \\ 1 & 2.23898 & -1.01689 & 0.00000 \\ 1 & -0.75398 & -1.96913 & 0.00000 \\ 1 & -2.33587 & -1.23710 & 0.00000\end{array}$

$E=-357.9540472832 \mathrm{au}$

ZPVE $=0.0607566793 \mathrm{au}$
Formamide $\mathbf{9}\left(C_{s}\right)$

$\begin{array}{lrrr}6 & -0.08889 & 0.40585 & 0.00000 \\ 8 & -1.13654 & -0.20753 & 0.00000 \\ 7 & 1.14685 & -0.16401 & 0.00000 \\ 1 & -0.03992 & 1.50720 & 0.00000 \\ 1 & 1.22515 & -1.16589 & 0.00000 \\ 1 & 1.97618 & 0.39886 & 0.00000 \\ & \end{array}$

cis-trans-Formimidic acid 10ct $\left(C_{s}\right)$

$\begin{array}{llll}6 & -0.09702 & 0.42191 & 0.00000\end{array}$

$\begin{array}{llll}7 & -1.22392 & -0.15094 & 0.00000\end{array}$

$\begin{array}{llll}8 & 1.10112 & -0.23239 & 0.00000\end{array}$

$\begin{array}{llll}1 & -0.03131 & 1.50810 & 0.00000\end{array}$

$\begin{array}{llll}1 & -1.09283 & -1.16437 & 0.00000\end{array}$

$\begin{array}{llll}1 & 1.80920 & 0.41817 & 0.00000\end{array}$

$E=-169.6195320195 \mathrm{au}$

ZPVE $=0.0457951554 \mathrm{au}$

trans-trans-Formimidic acid 10tt $\left(C_{\mathrm{s}}\right)$

$\begin{array}{llll}6 & -0.09521 & 0.41779 & 0.00000\end{array}$

$\begin{array}{llll}7 & -1.22593 & -0.15715 & 0.00000\end{array}$

$\begin{array}{llll}8 & 1.15060 & -0.12778 & 0.00000\end{array}$

$\begin{array}{llll}1 & -0.02669 & 1.50021 & 0.00000\end{array}$

$\begin{array}{llll}1 & -1.12639 & -1.17477 & 0.00000\end{array}$

$\begin{array}{llll}1 & 1.05932 & -1.08850 & 0.00000\end{array}$

$E=-169.6203742902 \mathrm{au}$

ZPVE $=0.0459600931 \mathrm{au}$ 


$$
\begin{aligned}
& \text { trans-cis-Formimidic acid 10tc }\left(C_{s}\right) \\
& \begin{array}{llll}
7 & 1.14612 & 0.27991 & 0.00000
\end{array} \\
& \begin{array}{llll}
6 & 0.09335 & -0.42877 & 0.00000
\end{array} \\
& \begin{array}{llll}
1 & 1.96556 & -0.32038 & 0.00000
\end{array} \\
& \begin{array}{llll}
8 & -1.13646 & 0.12415 & 0.00000
\end{array} \\
& \begin{array}{llll}
1 & 0.02639 & -1.51491 & 0.00000
\end{array} \\
& \begin{array}{lllll}
1 & -0.99169 & 1.08104 & 0.00000
\end{array} \\
& E=-169.6253727 \mathrm{au} \\
& \text { ZPVE }=0.046281204 \mathrm{au}
\end{aligned}
$$

cis-cis-Formimidic acid $10 \mathrm{cc}\left(C_{\mathrm{s}}\right)$

$$
\begin{array}{rrrr}
7 & 1.16366 & 0.26752 & 0.00000 \\
6 & 0.09653 & -0.41311 & 0.00000 \\
1 & 1.95654 & -0.36835 & 0.00000 \\
8 & -1.10313 & 0.22159 & 0.00000 \\
1 & 0.03032 & -1.50405 & 0.00000 \\
1 & -1.79717 & -0.44249 & 0.00000
\end{array}
$$$$
E=-169.6165706 \mathrm{au}
$$$$
\text { ZPVE }=0.045828462 \mathrm{au}
$$

Oxalic acid monoamide - enolimine tautomer a 12a $\left(C_{s}\right)$

$$
\begin{array}{cccc}
7 & -1.45387 & 1.16350 & 0.00000 \\
6 & -0.76804 & 0.09625 & 0.00000 \\
1 & -0.81176 & 1.95524 & 0.00000 \\
8 & -1.32308 & -1.13000 & 0.00000 \\
6 & 0.74801 & 0.09134 & 0.00000 \\
1 & -2.27880 & -0.98024 & 0.00000 \\
8 & 1.38924 & 1.11388 & 0.00000 \\
8 & 1.27552 & -1.14058 & 0.00000 \\
1 & 2.23608 & -1.01719 & 0.00000
\end{array}
$$

$E=-357.9395336236 a u$ ZPVE $=0.0616977007 \mathrm{au}$
Oxalic acid monoamide - enolimine tautomer b $\mathbf{1 2 b}\left(C_{s}\right)$

$\begin{array}{cccc}7 & -1.46845 & 1.16582 & 0.00000 \\ 6 & -0.75680 & 0.12098 & 0.00000 \\ 1 & -0.84417 & 1.97256 & 0.00000 \\ 6 & 0.76492 & 0.09864 & 0.00000 \\ 8 & -1.29017 & -1.12828 & 0.00000 \\ 8 & 1.40366 & 1.11619 & 0.00000 \\ 8 & 1.32259 & -1.12142 & 0.00000 \\ 1 & -2.25076 & -1.02071 & 0.00000 \\ 1 & 0.60985 & -1.77556 & 0.00000\end{array}$

$E=-357.9393592101 \mathrm{au}$ ZPVE $=0.0616650319 \mathrm{au}$

Oxalic acid monoamide - enolimine tautomer $\mathrm{c} 12 \mathrm{c}\left(C_{\mathrm{s}}\right)$

$\begin{array}{rrrr}7 & -1.41977 & 1.23165 & 0.00000 \\ 6 & -0.77328 & 0.13613 & 0.00000 \\ 1 & -0.80776 & 2.04283 & 0.00000 \\ 8 & -1.42573 & -1.03252 & 0.00000 \\ 6 & 0.74269 & -0.08386 & 0.00000 \\ 1 & -0.73667 & -1.71737 & 0.00000 \\ 8 & 1.18392 & -1.20710 & 0.00000 \\ 8 & 1.54165 & 0.98882 & 0.00000 \\ 1 & 1.00598 & 1.79020 & 0.00000\end{array}$

$E=-357.9290977406 \mathrm{au}$ ZPVE $=0.0610739653 \mathrm{au}$ 
Oxalic acid monoamide - enolimine tautomer $\mathrm{d} \mathbf{1 2 d}\left(C_{1}\right)$

$\begin{array}{lrrr}7 & -1.38675 & 1.23133 & -0.15643 \\ 6 & -0.75613 & 0.15471 & 0.01571 \\ 1 & -0.69418 & 1.98286 & -0.24301 \\ 8 & -1.42135 & -1.05663 & 0.08737 \\ 6 & 0.77112 & 0.07138 & 0.01979 \\ 1 & -1.42947 & -1.35303 & 1.00584 \\ 8 & 1.46434 & 1.04498 & 0.15159 \\ 8 & 1.26210 & -1.16509 & -0.15996 \\ 1 & 0.50047 & -1.75466 & -0.26588\end{array}$

$E=-357.9233506742 \mathrm{au}$

ZPVE $=0.0604201107 \mathrm{au}$

Oxalic acid monoamide - enolimine tautomer e $12 \mathrm{e}\left(C_{\mathrm{s}}\right)$

$\begin{array}{rrrr}7 & -1.33590 & 1.25039 & 0.00000 \\ 6 & -0.75902 & 0.11856 & 0.00000 \\ 1 & -0.63800 & 1.98925 & 0.00000 \\ 8 & -1.44239 & -1.03857 & 0.00000 \\ 1 & -2.37579 & -0.78529 & 0.00000 \\ 6 & 0.73700 & -0.12984 & 0.00000 \\ 8 & 1.25540 & -1.21398 & 0.00000 \\ 8 & 1.41474 & 1.03993 & 0.00000 \\ 1 & 2.35231 & 0.80204 & 0.00000\end{array}$

$E=-357.9379539216 a u$ ZPVE $=0.0616184988 \mathrm{au}$
Oxalic acid monoamide - enolimine tautomer $\mathrm{f} \mathbf{1 2 f}\left(C_{s}\right)$

$\begin{array}{rrrr}7 & -1.35486 & 1.26635 & 0.00000 \\ 6 & -0.77444 & 0.13821 & 0.00000 \\ 1 & -0.64684 & 1.99688 & 0.00000 \\ 8 & -1.46866 & -1.01300 & 0.00000 \\ 1 & -0.81247 & -1.72584 & 0.00000 \\ 6 & 0.72303 & -0.10198 & 0.00000 \\ 8 & 1.19476 & -1.21681 & 0.00000 \\ 8 & 1.44108 & 1.02873 & 0.00000 \\ 1 & 2.37217 & 0.76462 & 0.00000\end{array}$

$E=-357.9385470780 \mathrm{au}$ ZPVE $=0.0619002474 \mathrm{au}$

Oxalic acid monoamide - enolimine tautomer g $12 \mathrm{~g}\left(C_{s}\right)$

$\begin{array}{cccc}7 & -1.46508 & 1.17109 & 0.00000 \\ 6 & -0.78209 & 0.10377 & 0.00000 \\ 1 & -0.81116 & 1.95380 & 0.00000 \\ 8 & -1.37951 & -1.10624 & 0.00000 \\ 1 & -0.69242 & -1.78198 & 0.00000 \\ 6 & 0.73697 & 0.11394 & 0.00000 \\ 8 & 1.40478 & 1.11426 & 0.00000 \\ 8 & 1.24668 & -1.14105 & 0.00000 \\ 1 & 2.21032 & -1.05370 & 0.00000\end{array}$

$E=-357.9356991509 \mathrm{au}$ ZPVE $=0.0615314881 \mathrm{au}$ 
Oxalic acid monoamide - enolimine tautomer h $\mathbf{1 2 h}\left(C_{1}\right)$

$\begin{array}{rrrc}7 & -1.36400 & -1.16694 & 0.36075 \\ 6 & -0.75345 & -0.10121 & 0.02124 \\ 1 & -0.70950 & -1.88474 & 0.66209 \\ 6 & 0.75360 & 0.11863 & 0.02416 \\ 8 & -1.41287 & 1.00283 & -0.35356 \\ 8 & 1.26772 & 1.14525 & 0.36198 \\ 8 & 1.47676 & -0.96761 & -0.33916 \\ 1 & -2.35380 & 0.79164 & -0.26856 \\ 1 & 0.87979 & -1.63548 & -0.69729\end{array}$

$E=-357.9281551112 \mathrm{au}$

ZPVE $=0.0614097370 \mathrm{au}$

Oxalic acid monoamide - enolimine tautomer i $\mathbf{1 2} \mathbf{i}\left(C_{1}\right)$

$\begin{array}{rrrr}7 & 1.37961 & 1.20028 & 0.21908 \\ 6 & 0.77213 & 0.10653 & 0.00809 \\ 1 & 2.39223 & 1.06912 & 0.21457 \\ 8 & 1.28157 & -1.13246 & -0.21375 \\ 6 & -0.74626 & 0.11214 & -0.01419 \\ 1 & 2.24374 & -1.06849 & -0.18587 \\ 8 & -1.40903 & 1.09444 & -0.21401 \\ 8 & -1.25238 & -1.11366 & 0.22629 \\ 1 & -2.21368 & -1.00342 & 0.19732\end{array}$

$E=-357.9282332434 \mathrm{au}$

ZPVE $=0.06096831 \mathrm{au}$
Oxalic acid monoamide - enolimine tautomer j 12j $\left(C_{s}\right)$

$\begin{array}{rrrr}7 & 1.18839 & 1.30910 & 0.00000 \\ 6 & 0.75671 & 0.11393 & 0.00000 \\ 1 & 2.20613 & 1.34779 & 0.00000 \\ 8 & 1.47097 & -1.01742 & 0.00000 \\ 1 & 0.81338 & -1.73506 & 0.00000 \\ 6 & -0.74431 & -0.10784 & 0.00000 \\ 8 & -1.20008 & -1.22744 & 0.00000 \\ 8 & -1.46026 & 1.00949 & 0.00000 \\ 1 & -0.80310 & 1.73209 & 0.00000\end{array}$

$E=-357.9470239405 \mathrm{au}$ ZPVE $=0.0622905203 \mathrm{au}$

Oxalic acid monoamide - enolimine tautomer k $12 \mathrm{k}\left(C_{\mathrm{s}}\right)$
$\begin{array}{llll}7 & 1.18839 & 1.30910 & 0.00000\end{array}$
$\begin{array}{llll}6 & 0.75671 & 0.11393 & 0.00000\end{array}$
$\begin{array}{llll}1 & 2.20613 & 1.34779 & 0.00000\end{array}$
$\begin{array}{llll}8 & 1.47097 & -1.01742 & 0.00000\end{array}$
$\begin{array}{llll}6 & -0.74431 & -0.10784 & 0.00000\end{array}$
$\begin{array}{llll}1 & 0.81338 & -1.73506 & 0.00000\end{array}$
$\begin{array}{llll}8 & -1.20008 & -1.22744 & 0.00000\end{array}$
$\begin{array}{llll}8 & -1.46026 & 1.00949 & 0.00000\end{array}$
$\begin{array}{llll}1 & -0.80310 & 1.73209 & 0.00000\end{array}$

$E=-357.9470239405 \mathrm{au}$ ZPVE $=0.0622905203 \mathrm{au}$ 
Oxalic acid monoamide - enolimine tautomer | 12I $\left(C_{s}\right)$

$\begin{array}{rrrr}7 & 1.22842 & 1.31017 & 0.00000 \\ 6 & 0.76254 & 0.12824 & 0.00000 \\ 1 & 2.25030 & 1.30632 & 0.00000 \\ 8 & 1.42130 & -1.05525 & 0.00000 \\ 1 & 2.36723 & -0.86819 & 0.00000 \\ 6 & -0.73512 & -0.11429 & 0.00000 \\ 8 & -1.23003 & -1.21270 & 0.00000 \\ 8 & -1.42954 & 1.03451 & 0.00000 \\ 1 & -2.35983 & 0.76735 & 0.00000\end{array}$

$E=-357.9296520979 \mathrm{au}$

ZPVE $=0.0610900606 \mathrm{au}$

Oxalic acid monoamide - enolimine tautomer m $12 \mathrm{~m}\left(C_{\mathrm{s}}\right)$

$\begin{array}{rrrr}7 & 1.39751 & 1.22307 & 0.00000 \\ 6 & 0.76299 & 0.12922 & 0.00000 \\ 1 & 2.40822 & 1.07701 & 0.00000 \\ 8 & 1.25858 & -1.15092 & 0.00000 \\ 1 & 2.22185 & -1.11974 & 0.00000 \\ 6 & -0.76550 & 0.11870 & 0.00000 \\ 8 & -1.41906 & 1.12073 & 0.00000 \\ 8 & -1.31494 & -1.11270 & 0.00000 \\ 1 & -0.60179 & -1.76434 & 0.00000\end{array}$

$E=-357.9265175710 a u$ ZPVE $=0.0608594666 \mathrm{au}$
Oxalic acid monoamide - enolimine tautomer $\mathrm{n} \mathbf{1 2 n}\left(C_{\mathrm{s}}\right)$
$\begin{array}{llll}7 & 1.26771 & 1.30565 & 0.00000\end{array}$
$\begin{array}{lllll}6 & 0.76857 & 0.14054 & 0.00000\end{array}$
$\begin{array}{lllll}1 & 2.28694 & 1.23721 & 0.00000\end{array}$
$\begin{array}{llll}8 & 1.45185 & -1.02433 & 0.00000\end{array}$
$\begin{array}{llll}1 & 0.78139 & -1.72672 & 0.00000\end{array}$
$\begin{array}{llll}6 & -0.72718 & -0.08339 & 0.00000\end{array}$
$\begin{array}{llll}8 & -1.18234 & -1.20908 & 0.00000\end{array}$
$\begin{array}{llll}8 & -1.45381 & 1.03126 & 0.00000\end{array}$
$\begin{array}{llll}1 & -2.37954 & 0.74691 & 0.00000\end{array}$

$E=-357.9398567358 \mathrm{au}$

ZPVE $=0.0617861456 \mathrm{au}$

Oxalic acid monoamide - enolimine tautomer o $120\left(C_{s}\right)$

$\begin{array}{rrrr}7 & 1.40363 & 1.21282 & 0.00000 \\ 6 & 0.77546 & 0.11321 & 0.00000 \\ 1 & 2.40772 & 1.02546 & 0.00000 \\ 8 & 1.35583 & -1.11398 & 0.00000 \\ 6 & -0.74262 & 0.13660 & 0.00000 \\ 1 & 0.65716 & -1.77963 & 0.00000 \\ 8 & -1.43243 & 1.11555 & 0.00000 \\ 8 & -1.23165 & -1.13652 & 0.00000 \\ 1 & -2.19559 & -1.05921 & 0.00000\end{array}$

$E=-357.9337225025 \mathrm{au}$

ZPVE $=0.0611761151 \mathrm{au}$ 
Oxalic acid monoamide - enolimine tautomer $p \operatorname{12p}\left(C_{s}\right)$

$\begin{array}{rrrr}7 & 1.16089 & 1.30807 & 0.00000 \\ 6 & 0.75213 & 0.10100 & 0.00000 \\ 1 & 2.17577 & 1.39663 & 0.00000 \\ 8 & 1.44960 & -1.04607 & 0.00000 \\ 1 & 2.39139 & -0.83671 & 0.00000 \\ 6 & -0.75619 & -0.13507 & 0.00000 \\ 8 & -1.25208 & -1.22749 & 0.00000 \\ 8 & -1.44918 & 1.01018 & 0.00000 \\ 1 & -0.78370 & 1.72180 & 0.00000\end{array}$

$E=-357.9350488822 \mathrm{au}$ ZPVE $=0.0616564265 \mathrm{au}$

TS1: 1c $\rightarrow$ 10tt $\left(C_{1}\right)$

$8-1.13643-0.12181-0.01853$

$\begin{array}{lllll}1 & -0.98729 & -1.08768 & -0.01665\end{array}$

$\begin{array}{llll}6 & 0.04015 & 0.50187 & 0.06246\end{array}$

$\begin{array}{llll}7 & 1.18365 & -0.18772 & 0.03203\end{array}$

$\begin{array}{lllll}1 & 1.07877 & -1.19797 & -0.19705\end{array}$

$\begin{array}{lllll}1 & 1.02041 & 0.85143 & -0.68083\end{array}$

$E=-169.4988023454 \mathrm{au}$

ZPVE $=0.0389369337 \mathrm{au}$

$v_{\mathrm{i}}=1876.7300 i \mathrm{~cm}^{-1}$

TS2: $1 \mathrm{c} \rightarrow \mathrm{HNCO}+\mathrm{H}_{2}\left(C_{s}\right)$

$\begin{array}{cccc}8 & -1.08138 & 0.10013 & 0.00000 \\ 6 & -0.01888 & -0.49766 & 0.00000 \\ 7 & 1.09315 & 0.12655 & 0.00000 \\ 1 & 2.03545 & -0.22813 & 0.00000 \\ 1 & -0.44202 & 1.47944 & 0.00000 \\ 1 & 0.60503 & 1.32673 & 0.00000\end{array}$

$E=-169.5016841490 \mathrm{au}$

ZPVE $=0.033719165 \mathrm{au}$

$v_{\mathrm{i}}=1823.8533 i \mathrm{~cm}^{-1}$
TS3: $1 \mathrm{c} \rightarrow \mathrm{NH}_{3}+\mathrm{CO}\left(C_{s}\right)$

$\begin{array}{cccc}8 & -1.01069 & 0.29888 & 0.00000 \\ 6 & -0.22784 & -0.69560 & 0.00000 \\ 7 & 1.10585 & 0.18301 & 0.00000 \\ 1 & 1.67951 & 0.02009 & 0.82448 \\ 1 & 1.67951 & 0.02009 & -0.82448 \\ 1 & 0.02904 & 0.95590 & 0.00000\end{array}$

$E=-169.5157844219 \mathrm{au}$

ZPVE $=0.03915191 \mathrm{au}$

$v_{\mathrm{i}}=1771.4166 i \mathrm{~cm}^{-1}$

TS4: (valley ridge inflection point) $1 \mathrm{c} \rightarrow$

$\mathrm{NH}_{3}+\mathrm{CO}\left(C_{\mathrm{s}}\right)$

$\begin{array}{llll}8 & -1.09642 & -0.20135 & 0.00000\end{array}$

$\begin{array}{llll}1 & -0.75708 & -1.12449 & 0.00000\end{array}$

$\begin{array}{llll}6 & -0.07131 & 0.61575 & 0.00000\end{array}$

$\begin{array}{lllll}7 & 1.14973 & -0.14416 & 0.00000\end{array}$

$\begin{array}{llll}1 & 1.51621 & -0.50432 & -0.86297\end{array}$

$\begin{array}{llll}1 & 1.51621 & -0.50432 & 0.86297\end{array}$

$E=-169.5239532932 \mathrm{au}$

ZPVE $=0.0418517895 \mathrm{au}$

$v_{\mathrm{i}}=829.9754 i \mathrm{~cm}^{-1}$

TS5: 1c $\rightarrow$ 1t $\left(C_{1}\right)$

$\begin{array}{llll}8 & -1.10356 & -0.17303 & -0.06262\end{array}$

$\begin{array}{llll}1 & -1.30891 & -0.49605 & 0.82162\end{array}$

$\begin{array}{lllll}6 & 0.05376 & 0.60774 & 0.01882\end{array}$

$\begin{array}{lllll}7 & 1.09236 & -0.21233 & 0.00099\end{array}$

$\begin{array}{lllll}1 & 0.98571 & -1.22047 & -0.08155\end{array}$

$\begin{array}{llll}1 & 2.01976 & 0.17669 & 0.01583\end{array}$

$E=-169.5581021579 a u$

ZPVE $=0.0438194087 \mathrm{au}$

$v_{\mathrm{i}}=681.0818 i \mathrm{~cm}^{-1}$ 
TS6: 1t $\rightarrow \mathrm{HNC}+\mathrm{H}_{2} \mathrm{O}\left(C_{1}\right)$

$\begin{array}{rrrr}8 & -1.08336 & 0.16546 & -0.05887 \\ 1 & -1.58211 & -0.01028 & 0.75084 \\ 6 & 0.26297 & -0.72099 & -0.00573 \\ 1 & -0.16021 & 0.95357 & 0.04305 \\ 7 & 0.99351 & 0.34271 & 0.01550 \\ 1 & 2.00067 & 0.25385 & -0.00671\end{array}$

$E=-169.5037054255 \mathrm{au}$

ZPVE $=0.03887797 \mathrm{au}$

$v_{\mathrm{i}}=1744.6268 i \mathrm{~cm}^{-1}$

TS7: 1t $\rightarrow$ 10ct $\left(C_{1}\right)$

$\begin{array}{rrrr}8 & -1.08416 & 0.22416 & 0.02075 \\ 1 & -1.82311 & -0.39205 & -0.00461 \\ 6 & 0.04652 & -0.50946 & -0.06672 \\ 7 & 1.18452 & 0.18365 & -0.03049 \\ 1 & 0.98403 & -0.83959 & 0.72194 \\ 1 & 1.03349 & 1.18834 & 0.17132\end{array}$

$E=-169.5045007928 \mathrm{au}$

ZPVE $=0.0397286347 \mathrm{au}$

$v_{\mathrm{i}}=1868.1577 i \mathrm{~cm}^{-1}$

TS8: 1c $\rightarrow$ 10tt $\left(C_{s}\right)$

$\begin{array}{llll}8 & -1.12119 & 0.24104 & 0.00000\end{array}$

$\begin{array}{llll}1 & -1.13626 & -0.98486 & 0.00000\end{array}$

$\begin{array}{llll}6 & -0.01947 & -0.48373 & 0.00000\end{array}$

$\begin{array}{lllll}7 & 1.15091 & 0.15175 & 0.00000\end{array}$

$\begin{array}{llll}1 & 1.15526 & 1.16524 & 0.00000\end{array}$

$\begin{array}{llll}1 & 2.01587 & -0.35465 & 0.00000\end{array}$

$E=-169.5206629339 \mathrm{au}$

ZPVE $=0.0393453737 \mathrm{au}$

$v_{\mathrm{i}}=2035.4273 i \mathrm{~cm}^{-1}$
TS9: $1 \mathrm{t}-\mathrm{CO}_{2} \rightarrow 2 \mathrm{c}\left(C_{\mathrm{s}}\right)$

$\begin{array}{rrrr}6 & 0.89573 & -0.12281 & 0.00000 \\ 7 & 1.89406 & -0.98584 & 0.00000 \\ 8 & 1.31233 & 1.13065 & 0.00000 \\ 1 & 1.68059 & -1.96672 & 0.00000 \\ 1 & 2.86456 & -0.69735 & 0.00000 \\ 1 & 0.48084 & 1.64226 & 0.00000 \\ 8 & -1.34832 & 1.12156 & 0.00000 \\ 6 & -1.26171 & -0.07091 & 0.00000 \\ 8 & -1.66432 & -1.17942 & 0.00000\end{array}$

$E=-357.9129309375 a u$ ZPVE $=0.0593557446 \mathrm{au}$ $v_{\mathrm{i}}=247.0041 i \mathrm{~cm}^{-1}$

TS10: $1 \mathrm{c}-\mathrm{CO}_{2} \rightarrow 1 \mathrm{t}-\mathrm{CO}_{2}\left(C_{1}\right)$

$\begin{array}{rrrc}6 & 1.22163 & 0.19876 & 0.01302 \\ 7 & 1.44250 & -1.10254 & -0.00993 \\ 8 & 2.45240 & 0.85476 & 0.05552 \\ 1 & 0.64813 & -1.72221 & -0.00468 \\ 1 & 2.38006 & -1.49424 & 0.02042 \\ 1 & 2.79664 & 0.90762 & -0.84295 \\ 6 & -1.79349 & 0.04708 & -0.00066 \\ 8 & -1.58212 & -1.10446 & 0.00204 \\ 8 & -2.07113 & 1.17598 & -0.00602\end{array}$

$E=-357.8927671151 \mathrm{au}$

ZPVE $=0.056591325 \mathrm{au}$

$v_{\mathrm{i}}=682.1332 i \mathrm{~cm}^{-1}$ 
TS11: $2 \mathrm{t} \rightarrow \mathbf{2 c}\left(C_{1}\right)$

$\begin{array}{rrrr}8 & -1.22434 & 1.26049 & -0.14293 \\ 6 & -0.77986 & 0.13854 & -0.00277 \\ 6 & 0.73711 & -0.14245 & -0.00929 \\ 8 & 1.50296 & 0.96187 & 0.20022 \\ 8 & 1.17808 & -1.25272 & -0.15393 \\ 7 & -1.50145 & -0.99713 & 0.15368 \\ 1 & -1.02772 & -1.88231 & 0.11788 \\ 1 & -2.50065 & -0.95062 & 0.06243 \\ 1 & 1.78034 & 1.34501 & -0.63812\end{array}$

$E=-357.9397543157 \mathrm{au}$

ZPVE $=0.059609924 \mathrm{au}$

$v_{\mathrm{i}}=635.9944 i \mathrm{~cm}^{-1}$

TS12: $12 b \rightarrow 2 c\left(C_{s}\right)$

$\begin{array}{llll}7 & -1.56673 & 1.01180 & 0.00000\end{array}$

$\begin{array}{llll}6 & -0.69931 & 0.04937 & 0.00000\end{array}$

$\begin{array}{llll}1 & -1.32094 & 1.99217 & 0.00000\end{array}$

$\begin{array}{llll}6 & 0.81135 & 0.11802 & 0.00000\end{array}$

$\begin{array}{llll}8 & -1.32725 & -1.08342 & 0.00000\end{array}$

$\begin{array}{lllll}8 & 1.41343 & 1.15686 & 0.00000\end{array}$

$\begin{array}{lllll}8 & 1.38445 & -1.09169 & 0.00000\end{array}$

$\begin{array}{lllll}1 & 0.66736 & -1.74635 & 0.00000\end{array}$

$\begin{array}{llll}1 & -2.25179 & -0.13674 & 0.00000\end{array}$

$E=-357.8809819710$ au

ZPVE $=0.0563447946 \mathrm{au}$

$v_{\mathrm{i}}=2034.8473 i \mathrm{~cm}^{-1}$
TS13: 12a $\rightarrow$ 2t $\left(C_{s}\right)$

$\begin{array}{rrrr}7 & -1.53529 & 1.00969 & 0.00000 \\ 6 & -0.71655 & 0.00442 & 0.00000 \\ 1 & -1.25307 & 1.98047 & 0.00000 \\ 8 & -1.38493 & -1.08680 & 0.00000 \\ 6 & 0.79279 & 0.10373 & 0.00000 \\ 8 & 1.36807 & 1.15746 & 0.00000 \\ 8 & 1.37915 & -1.09402 & 0.00000 \\ 1 & 2.33786 & -0.95307 & 0.00000 \\ 1 & -2.28127 & -0.10277 & 0.00000\end{array}$

$E=-357.8785112778 \mathrm{au}$

ZPVE $=0.0561626460 \mathrm{au}$

$v_{\mathrm{i}}=2024.6662 i \mathrm{~cm}^{-1}$

TS14: 10tc $\rightarrow 9\left(C_{s}\right)$

$\begin{array}{rrrr}7 & 1.05287 & 0.24990 & 0.00000 \\ 6 & 0.00231 & -0.52394 & 0.00000 \\ 1 & 2.01129 & -0.06699 & 0.00000 \\ 8 & -1.04782 & 0.21402 & 0.00000 \\ 1 & -0.03478 & -1.60954 & 0.00000 \\ 1 & -0.00336 & 1.04628 & 0.00000\end{array}$

$E=-169.5693474 \mathrm{au}$

$\mathrm{ZPVE}=0.040687504 \mathrm{au}$

$v_{\mathrm{i}}=1990.5345 i \mathrm{~cm}^{-1}$

TS15: 10cc $\rightarrow$ 10tc $\left(C_{1}\right)$

$\begin{array}{llll}7 & 1.15856 & -0.28809 & 0.00318\end{array}$

$\begin{array}{lllll}6 & 0.10931 & 0.41972 & 0.00942\end{array}$

$\begin{array}{llll}1 & 1.96692 & 0.33275 & 0.00882\end{array}$

$\begin{array}{llll}8 & -1.13243 & -0.16192 & -0.06348\end{array}$

$\begin{array}{lllll}1 & 0.08462 & 1.51195 & 0.01501\end{array}$

$\begin{array}{llll}1 & -1.47813 & -0.26970 & 0.82727\end{array}$

$E=-169.6085431 \mathrm{au}$

ZPVE $=0.044642185 \mathrm{au}$

$v_{\mathrm{i}}=500.1411 i \mathrm{~cm}^{-1}$ 


\begin{tabular}{|c|c|c|c|c|c|}
\hline \multicolumn{3}{|c|}{ TS16: $10 \mathrm{tt} \rightarrow 10 \mathrm{tc}\left(C_{\mathrm{s}}\right)$} & \multicolumn{3}{|c|}{ TS18: $10 \mathrm{ct} \rightarrow 10 \mathrm{tt}\left(C_{1}\right)$} \\
\hline $6 \quad 0.09547$ & 0.42327 & 0.00000 & $7-1.22214$ & -0.17604 & -0.00194 \\
\hline $8-1.15495$ & -0.14677 & 0.00000 & $6-0.11224$ & 0.43026 & -0.01475 \\
\hline $1-1.00254$ & -1.10136 & 0.00000 & $1-1.05370$ & -1.18391 & 0.04057 \\
\hline $1-0.04044$ & 1.50917 & 0.00000 & 81.13639 & -0.16412 & 0.06407 \\
\hline $7 \quad 1.16725$ & -0.17581 & 0.00000 & $1-0.08332$ & 1.51696 & -0.03165 \\
\hline 12.01786 & -0.67556 & 0.00000 & 11.41900 & -0.40545 & -0.82315 \\
\hline \multicolumn{3}{|c|}{$E=-169.5839548 a u$} & \multicolumn{3}{|c|}{$E=-169.6102226 \mathrm{au}$} \\
\hline \multicolumn{3}{|c|}{ ZPVE $=0.043239975 \mathrm{au}$} & \multicolumn{3}{|c|}{ ZPVE $=0.044617803 \mathrm{au}$} \\
\hline \multicolumn{3}{|c|}{$v_{\mathrm{i}}=1086.8358 i \mathrm{~cm}^{-1}$} & \multicolumn{3}{|c|}{$v_{\mathrm{i}}=466.8502 i \mathrm{~cm}^{-1}$} \\
\hline \multicolumn{3}{|c|}{ TS17: $10 \mathrm{cc} \rightarrow 10 \mathrm{ct}\left(C_{\mathrm{s}}\right)$} & \multicolumn{3}{|c|}{ TS19: $\mathrm{HNC} \rightarrow \mathrm{HCN}\left(C_{s}\right)$} \\
\hline $6-0.09969$ & 0.41227 & 0.00000 & $7-0.55563$ & -0.02148 & 0.00000 \\
\hline $8 \quad 1.11568$ & -0.24527 & 0.00000 & 60.62345 & -0.06542 & 0.00000 \\
\hline 11.80623 & 0.42637 & 0.00000 & 10.29680 & 1.07742 & 0.00000 \\
\hline 10.04213 & 1.50497 & 0.00000 & & & \\
\hline $7-1.17408$ & -0.16816 & 0.00000 & $E=-93.198$ & 7056 au & \\
\hline $0-1.64725$ & 0.47010 & 0.00000 & $Z P V E=0.01$ & $070725 \mathrm{au}$ & \\
\hline $1-2.05492$ & -0.61100 & 0.00000 & $v_{\mathrm{i}}=1194.64$ & $51 i \mathrm{~cm}^{-1}$ & \\
\hline \multicolumn{6}{|c|}{$E=-169.5781499 \mathrm{au}$} \\
\hline \multicolumn{6}{|c|}{ ZPVE $=0.042951852 \mathrm{au}$} \\
\hline \multicolumn{6}{|c|}{$v_{\mathrm{i}}=1075.5197 i \mathrm{~cm}^{-1}$} \\
\hline
\end{tabular}




\section{References}

(1) Maier, G.; Endres, J.; Reisenauer, H. P., Interconversions Between Oxalic Acid Monoamide Rotamers: Photochemical Process Versus Tunneling. J. Mol. Struct. 2012, 1025, 2-5.

(2) Jones, E.; Oliphant, T.; Peterson, P., SciPy: Open Source Scientific Tools for Python. SciPy: Open source scientific tools for Python. 2001.

(3) Hunter, J. D., Matplotlib: A 2D Graphics Environment. Comput. Sci. Eng. 2007, 9, 90-95.

(4) Harris, C. R.; Millman, K. J.; van der Walt, S. J.; Gommers, R.; Virtanen, P.; Cournapeau, D.; Wieser, E.; Taylor, J.; Berg, S.; Smith, N. J.; Kern, R.; Picus, M.; Hoyer, S.; van Kerkwijk, M. H.; Brett, M.; Haldane, A.; del Río, J. F.; Wiebe, M.; Peterson, P.; Gérard-Marchant, P.; Sheppard, K.; Reddy, T.; Weckesser, W.; Abbasi, H.; Gohlke, C.; Oliphant, T. E., Array Programming with NumPy. Nature 2020, 585, 357-362.

(5) Rossum, G. V.; Drake, F. L., Python 3 Reference Manual. Python 3 Reference Manual. 2009.

(6) Bernhardt, B.; Ruth, M.; Eckhardt, A. K.; Schreiner, P. R., Ethynylhydroxycarbene (H$\mathrm{C} \equiv \mathrm{C}-\mathrm{C}-\mathrm{OH})$. J. Am. Chem. Soc. 2021, 143, 3741-3746.

(7) Becke, A. D., A New Mixing of Hartree-Fock and Local Density-Functional Theories. J. Chem. Phys. 1993, 98, 1372-1377.

(8) Lee, C.; Yang, W.; Parr, R. G., Development of the Colle-Salvetti Correlation-Energy Formula Into a Functional of the Electron Density. Phys. Rev. B 1988, 37, 785-789.

(9) McLean, A. D.; Chandler, G. S., Contracted Gaussian Basis Sets for Molecular Calculations. I. Second Row Atoms, Z=11-18. J. Chem. Phys. 1980, 72, 5639-5648.

(10) Krishnan, R.; Binkley, J. S.; Seeger, R.; Pople, J. A., Self-Consistent Molecular Orbital Methods. XX. A Basis Set for Correlated Wave Functions. J. Chem. Phys. 1980, 72, 650-654.

(11) Čížek, J., On the Correlation Problem in Atomic and Molecular Systems. Calculation of Wavefunction Components in Ursell-Type Expansion Using Quantum-Field Theoretical Methods. J. Chem. Phys. 1966, 45, 4256-4266.

(12) Noga, J.; Bartlett, R. J., The Full CCSDT Model for Molecular Electronic Structure. J. Chem. Phys. 1987, 86, 7041-7050.

(13) Raghavachari, K.; Trucks, G. W.; Pople, J. A.; Head-Gordon, M., A Fifth-Order Perturbation Comparison of Electron Correlation Theories. Chem. Phys. Lett. 1989, 157, 479483.

(14) Bartlett, R. J.; Watts, J. D.; Kucharski, S. A.; Noga, J., Non-Iterative Fifth-Order Triple and Quadruple Excitation Energy Corrections in Correlated Methods. Chem. Phys. Lett. 1990, $165,513-522$.

(15) Stanton, J. F., Why CCSD(T) Works: a Different Perspective. Chem. Phys. Lett. 1997, 281, 130-134.

(16) Wilson, A. K.; van Mourik, T.; Dunning, T. H., Gaussian Basis Sets for Use in Correlated Molecular Calculations. VI. Sextuple Zeta Correlation Consistent Basis Sets for Boron Through Neon. J. Mol. Struct. THEOCHEM 1996, 388, 339-349.

(17) Peterson, K. A.; Woon, D. E.; Jr., T. H. D., Benchmark Calculations With Correlated Molecular Wave Functions. IV. The Classical Barrier Height of the $\mathrm{H}+\mathrm{H}_{2} \rightarrow \mathrm{H}_{2}+\mathrm{H}$ Reaction. J. Chem. Phys. 1994, 100, 7410-7415.

(18) Woon, D. E.; Jr., T. H. D., Gaussian Basis Sets for Use in Correlated Molecular Calculations. III. The Atoms Aluminum Through Argon. J. Chem. Phys. 1993, 98, 1358-1371. 
(19) Kendall, R. A.; Jr., T. H. D.; Harrison, R. J., Electron Affinities of the First-Row Atoms Revisited. Systematic Basis Sets and Wave Functions. J. Chem. Phys. 1992, 96, 6796-6806.

(20) Jr., T. H. D., Gaussian Basis Sets for Use in Correlated Molecular Calculations. I. The Atoms Boron Through Neon and Hydrogen. J. Chem. Phys. 1989, 90, 1007-1023. 


\section{Full Citations for Electronic Structure Codes}

\section{Gaussian 16:}

M. J. Frisch, G. W. Trucks, H. B. Schlegel, G. E. Scuseria, M. A. Robb, J. R. Cheeseman, G. Scalmani, V. Barone, G. A. Petersson, H. Nakatsuji, X. Li, M. Caricato, A. V. Marenich, J. Bloino, B. G. Janesko, R. Gomperts, B. Mennucci, H. P. Hratchian, J. V. Ortiz, A. F. Izmaylov, J. L. Sonnenberg, D. Williams-Young, F. Ding, F. Lipparini, F. Egidi, J. Goings, B. Peng, A. Petrone, T. Henderson, D. Ranasinghe, V. G. Zakrzewski, J. Gao, N. Rega, G. Zheng, W. Liang, M. Hada, M. Ehara, K. Toyota, R. Fukuda, J. Hasegawa, M. Ishida, T. Nakajima, Y. Honda, O. Kitao, H. Nakai, T. Vreven, K. Throssell, J. A. Montgomery, Jr., J. E. Peralta, F. Ogliaro, M. J. Bearpark, J. J. Heyd, E. N. Brothers, K. N. Kudin, V. N. Staroverov, T. A. Keith, R. Kobayashi, J. Normand, K. Raghavachari, A. P. Rendell, J. C. Burant, S. S. Iyengar, J. Tomasi, M. Cossi, J. M. Millam, M. Klene, C. Adamo, R. Cammi, J. W. Ochterski, R. L. Martin, K. Morokuma, O. Farkas, J. B. Foresman, D. J. Fox, Gaussian 16 Revision C.01, 2016, Gaussian Inc., Wallingford.

CFOUR:

CFOUR, a quantum chemical program package written by J.F. Stanton, J. Gauss, L. Cheng, M. E. Harding, D. A. Matthews, P. G. Szalay with contributions from A. A. Auer, R. J. Bartlett, U. Benedikt, C. Berger, D. E. Bernholdt, Y. J. Bomble, O. Christiansen, F. Engel, R. Faber, M. Heckert, O. Heun, M. Hilgenberg, C. Huber, T.-C. Jagau, D. Jonsson, J. Jusélius, T. Kirsch, K. Klein, W. J. Lauderdale, F. Lipparini, T. Metzroth, L. A. Mück, D. P. O'Neill, D. R. Price, E. Prochnow, C. Puzzarini, K. Ruud, F. Schiffmann, W. Schwalbach, C. Simmons, S. Stopkowicz, A. Tajti, J. Vázquez, F. Wang, J. D. Watts and the integral packages MOLECULE (J. Almlöf and P. R. Taylor), PROPS (P. R. Taylor), ABACUS (T. Helgaker, H. J. Aa. Jensen, P. Jørgensen, and J. Olsen), and ECP routines by $A$. V. Mitin and $C$. van Wüllen. For the current version, see http://www.cfour.de.

\section{Polyrate:}

J. Zheng, J. L. Bao, R. Meana-Pañeda, S. Zhang, B. J. Lynch, J. C. Corchado, Y.-Y. Chuang, P. L. Fast, W.-P. Hu, Y.-P. Liu, G. C. Lynch, K. A. Nguyen, C. F. Jackels, A. Fernandez Ramos, B. A. Ellingson, V. S. Melissas, J. Villà, I. Rossi, E. L. Coitiño, J. Pu, T. V. Albu, A. Ratkiewicz, R. Steckler, B. C. Garrett, A. D. Isaacson, D. G. Truhlar, Polyrate Version 2017-C, 2017, University of Minnesota, Minneapolis.

NBO7:

E. D. Glendening, J, K. Badenhoop, A. E. Reed, J. E. Carpenter, J. A. Bohmann, C. M. Morales, P. Karafiloglou, C. R. Landis, F. Weinhold, NBO 7.0, 2018, Theoretical Chemistry Institute, University of Wisconsin, Madison. 